Michel Freitas Miudo de Oliveira

Estudos numéricos sobre a concomitância dos fenômenos de vibrações induzidas por vórtices (VIV) e de excitação paramétrica em cilindros rígidos montados em apoio elástico 
Michel Freitas Miudo de Oliveira

\title{
Estudos numéricos sobre a concomitância dos fenômenos de vibrações induzidas por vórtices (VIV) e de excitação paramétrica em cilindros rígidos montados em apoio elástico
}

\author{
Versão Corrigida
}

Dissertação apresentada à Escola Politécnica da Universidade de São Paulo para obtenção do título de Mestre em Ciências. Área de concentração: Engenharia de estruturas

Orientador: Prof. Dr. Guilherme Rosa Franzini

São Paulo 
Autorizo a reprodução e divulgação total ou parcial deste trabalho, por qualquer meio convencional ou eletrônico, para fins de estudo e pesquisa, desde que citada a fonte.

Este exemplar foi revisado e corrigido em relação à versão original, sob responsabilidade única do autor e com a anuência de seu orientador.

São Paulo, de de

Assinatura do autor:

Assinatura do orientador:

\section{Catalogação-na-publicação}

Oliveira, Michel Freitas Miudo de

Estudos numéricos sobre a concomitância dos fenômenos de vibrações induzidas por vórtices (VIV) e de excitação paramétrica em cilindros rígidos montados em apoio elástico / M. F. M. Oliveira -- versão corr. -- São Paulo, 2021.

$87 \mathrm{p}$.

Dissertação (Mestrado) - Escola Politécnica da Universidade de São Paulo. Departamento de Engenharia de Estruturas e Geotécnica.

1.Vibração induzida pela emissão de vórtices 2. Excitação paramétrica 3.Modelos fenomenológicos 4.Método das múltiplas escalas 5.Investigação numérica I.Universidade de São Paulo. Escola Politécnica. Departamento de Engenharia de Estruturas e Geotécnica II.t. 
Dedico este trabalho aos meus pais Otávio e Maria de Fátima, que me propuseram o melhor que eles poderiam me dar. Dedico também à minha namorada, Marcela, que é minha companheira muito paciente e amorosa 


\section{Agradecimentos}

Agradeço a Deus e à Nossa Senhora em primeiro lugar, por terem me propiciado saúde e força para chegar até aqui e realizar mais uma etapa de minha vida.

Agradeço a meus pais, Otavio Miudo de Oliveira e Maria de Fatima Freitas de Oliveira, por todo amor, carinho e atenção e por me incentivarem a continuar lutando por meus sonhos, sem nunca desistir, por maiores que fossem as dificuldades.

À minha namorada Marcela Rodrigues por todo amor e carinho, sempre me incentivando e me apoiando durante esse processo.

A todos da minha família em especial a meus avós, tios e primos.

Ao meu professor e orientador Prof. Dr. Guilherme Rosa Franzini, que desde o início do trabalho me apoiou muito e me incentivou a chegar até aqui. Sempre disposto a me acompanhar de perto, orientando meu trabalho da melhor maneira possível para que atingisse o melhor resultado, tanto na pesquisa quanto no aprendizado. Tenho que agradecer também pela paciência em me orientar, pois ao longo da jornada aconteceram coisas difíceis.

Agradeço a Escola Politécnica da Universidade de São Paulo e ao Laboratório de Mecânica Offshore, todos os colaboradores e Professores que de alguma forma contribuíram no desenvolvimento desta pesquisa.

Aos meus amigos de laboratório: Igor Mancilla, Renato Finotelli, João Cerqueira, Fernando Toni, Giovanni Amaral, Guilherme Vernizzi, Vitor Maciel, Vinícius Vaguetti, Tatiana Ueno, Daniela Kawaguti e Daniel Tomin. Por também terem feito parte do laboratório não poderia deixar de mencionar um grande amigo Mohammad Javad.

Aos professores Dr. Carlos Eduardo N. Mazzilli e Dr. Renato M. M. Orsino, que contribuíram com comentários que foram muito importantes no Exame de Qualificação.

Aos meus amigos de graduação: João Vitor, Thomas Hachul, Pamella Schiavon, Gabriel Sonoda, Arthur Nunes, Rodrigo Goulart, Pedro Amoroso, João Romani, Bruno Toledo, Vitor Chagas e Paula Bolelli, por todo período que estivemos juntos. Aqui vai um agradecimento especial à Laura Mazzoni que me ajudou em diversos momentos durante o período da graduação e continuou me ajudando nessa jornada acadêmica.

Aos meus professores de graduação Prof. M.E. Marcelo Gomes e Prof. M.E. Daniel Scheffer, que sempre me auxiliaram desde os tempos como aluno de graduação.

Aqui vai um agradecimento especial ao meu amigo Roberto Scalco (in memorian), petak, que foi meu professor e amigo desde o meu primeiro ano da graduação. Tenho 
certeza que você está em um lugar maravilhoso, pois durante sua passagem por esse mundo fez muitas coisas boas. Sempre disposto a ajudar quem quer que fosse e não media esforços para dar o seu melhor.

Dedico também às pessoas que não foram citadas, mas que de alguma forma auxiliaram para o sucesso deste trabalho. 
"Bom mesmo é ir à luta com determinação, abraçar a vida com paixão, perder com classe e vencer com ousadia, por que o mundo pertence a quem se atreve. E a vida é muito para ser insignificante." Charles Chaplin 


\section{Resumo}

As vibrações induzidas pela emissão vórtices (VIV) são um fenômeno de interação fluidoestrutura comum em vários problemas de engenharia. Outro fenômeno comumente encontrado em aplicações tecnológicas é a excitação paramétrica, que está associada à dependência do tempo de, ao menos, um dos parâmetros do modelo matemático. Quando a rigidez varia de forma harmônica com a frequência igual ao dobro do valor de uma das frequências naturais da estrutura, a configuração do equilíbrio trivial torna-se instável para o sistema linear não amortecido e surge instabilidade paramétrica. No contexto da dinâmica de risers, o VIV pode ocorrer concomitantemente ao fenômeno de excitação paramétrica devido à presença de correnteza marítima e modulação da rigidez geométrica, essa última associada aos movimentos da unidade flutuante no plano vertical. Este trabalho faz a investigação numérica da resposta de um cilindro rígido montado em base elástica com um e dois graus de liberdade sujeito à excitação concomitante pelo fenômeno de VIV e pela excitação paramétrica. As forças hidrodinâmicas são modeladas por meio de modelos fenomenológicos caracterizados pela equação de van der Pol. Curvas de amplitude característica em função da velocidade reduzida, ilustram o comportamento do sistema na região de lock-in. Esta pesquisa mostra que, ao contrário do observado para cilindros flexíveis, a excitação paramétrica amplifica a resposta VIV apenas em uma faixa estreita de velocidades reduzidas próximas ao pico de resposta do cilindro. Esse aumento na resposta do cilindro é acompanhado por um aumento nos coeficientes de força. Observou-se que existem algumas respostas moduladas em amplitude com rico conteúdo espectral. Esses últimos resultados são confirmados por um estudo analítico desenvolvido usando o método de escalas múltiplas. Também foi utilizada a Transformada de Hilbert-Huang, como análise no domínio do tempo-frequência e que é própria para utilização em sinais não estacionários. Destaca-se que a excitação paramétrica pode aumentar a amplitude da resposta da estrutura quando comparada ao caso somente do fenômeno de VIV.

Palavras-chave: Investigação numérica, Vibrações induzidas pela emissão de vórtices, Excitação paramétrica, modelos fenomenológicos, método das múltiplas escalas. 


\section{Abstract}

Vortex-induced vibration (VIV) is a fluid-structure interaction phenomenon that is very common in several engineering problems. Another phenomenon commonly found in technological applications is the parametric excitation, which is associated to a time-dependence of at least one of the parameters of the mathematical model. In the proposed problem, the stiffness is time-dependent. When the stiffness oscillates with twice the natural frequencies of the structure, the trivial equilibrium configuration becomes unstable for the linear undamped system and parametric instability occurs. In the context of riser's dynamics, VIV can occur concomitantly to the parametric excitation phenomenon due to the currents and geometric stiffness modulation, being the latter associated with the floating unity motions on the vertical plane. This work focuses on the numerical investigation of the response of a rigid cylinder assembled on to an elastic base subjected to concomitant excitation caused by VIV and parametric excitation phenomena. The hydrodynamic loads are modelled by means of phenomenological model characterized by the van der Pol equation. Characteristic amplitude curves as functions of the reduced velocity illustrate the behaviour of the system in the lock-in area. This research shows that, contrary to what was observed for flexible cylinders, parametric excitation amplifies the VIV response only in the range of reduced velocities close to the peak of the cylinder response. This increase in the cylinder response is accompanied by an increase in force coefficients. Note that there are some amplitude modulated responses with rich the amplitude spectra. These latter results are confirmed by an analytical study developed using the method of multiple scales (MMS). The Hilbert-Huang transform (HHT) is also employed as a time-frequency domain analyse suitable for use in non-stationary signals. It can be highlighted that the parametric excitation can increase the amplitude of the response when compared to the case in which only the VIV phenomenon is present.

Keywords: Numerical investigation, Vortex-Induced Vibrations, Parametric excitation, Wake-oscillator models, Method of multiple scales. 


\section{Lista de ilustrações}

Figura 1 - Representação de um modelo esquemático do riser flexível submetido ao VIV e a movimentos de topo. . . . . . . . . . . . . . . . . 19

Figura 2 - Regiões de escoamento perturbado pela presença de um corpo rombudo. 22

Figura 3 - Modelo bidimensional de formação e desprendimento de vórtices. . . . 22

Figura 4 - Representação do regime da esteira vorticosa em função do número de Reynolds. . . . . . . . . . . . . . . . . . 23

Figura 5 - Variação do número de Strouhal em função do número de Reynolds. . . 24

Figura 6 - Variação dos coeficientes de sustentação e de arrasto com o número de Reynolds. . . . . . . . . . . . . . . . . 26

Figura 7 - Estudo do fenômeno de VIV em cilindro rígido. . . . . . . . . . . . . 27

Figura 8 - Curva de amplitude de resposta em função da velocidade reduzida em cilindro rígido. . . . . . . . . . . . . . . . . . . . . . . . . 29

Figura 9 - Padrões de emissão de vórtices para cada ramo de resposta. . . . . . . 30

Figura 10 - Frequência adimensional de oscilação como função da velocidade reduzida. 30

Figura 11 - Curva de amplitude de resposta e frequência adimensional de oscilação em função da velocidade reduzida. VIV-2GL. . . . . . . . . . . . . . 32

Figura 12 - Curva de amplitude de resposta em função da velocidade reduzia. Comparação entre a resposta de um cilindro flexível $(* ;+)$ e cilindro rígido com dois graus de liberdade $($ o) . . . . . . . . . . . . . . . 33

Figura 13 - Representação da dedução de $C_{V Y}$. O deslocamento do cilindro é adotado para baixo. . . . . . . . . . . . . . . . 37

Figura 14 - Representação do fenômeno de VIV-2GL para um cilindro rígido montado sobre apoio elástico. . . . . . . . . . . . . . . . .

Figura 15 - Representação da decomposição das forças hidrodinâmicas em um cilindro submetido ao VIV-2GL. . . . . . . . . . . . . . . . . . . . . 40

Figura 16 - Diagrama de Strutt. . . . . . . . . . . . . . . . . . . 43

Figura 17 - Representação do modelo em estudo. A aceleração da gravidade é perpendicular ao plano xy. . . . . . . . . . . . . . . 46

Figura 18 - Curvas de respostas da amplitude característica . . . . . . . . . . . 53

Figura 19 - Envelopes de amplitude característica de oscilação. $U_{R}=4,99$. . . . 53

Figura 20 - Series temporais de resposta do cilindro para o caso sem excitação paramétrica . . . . . . . . . . . . . . . . 5 54

Figura 21 - Series temporais de resposta do cilindro para o caso $\delta_{y}=0,10$ e $n_{y}=1$. 55

Figura 22 - Espectro de Hilbert-Huang $\left(\delta_{y}=0,10\right.$ e $\left.n_{y}=1\right) \ldots \ldots$. . . . . . 55

Figura 23 - Curvas de respostas da amplitude característica . . . . . . . . . . 56

Figura 24 - Amplitude característica de oscilação direção na cross-wise . . . . . . . 57 
Figura 25 - Series temporais de resposta do cilindro para o caso $\delta_{y}=0,10$ e $n_{y}=2$. 58

Figura 26 - Series temporais de resposta do cilindro flexível . . . . . . . . . . . 58

Figura 27 - Series temporais de resposta do cilindro flexível . . . . . . . . . . . . 59

Figura 28 - Espectro de Hilbert-Huang $\left(\delta_{y}=0,10\right.$ e $\left.n_{y}=2\right) \ldots \ldots$. . . . . . . 60

Figura 29 - Series temporais de resposta do cilindro para o caso $\delta_{y}=0,10$ e $n_{y}=2 \quad 60$

Figura 30 - Series temporais de resposta do cilindro flexível . . . . . . . . . . . 61

Figura 31 - Espectro de Hilbert-Huang caso-2 $\left(\delta_{y}=0,10\right.$ e $\left.n_{y}=2\right) \ldots$. . . . . . . 62

Figura 32 - Mapa de amplitude para o caso de $\delta_{y}=0,10 \ldots \ldots$. . . . . . . . 62

Figura 33 - Influência de $\delta_{y}$ nos coeficientes de força em relação à velocidade reduzida

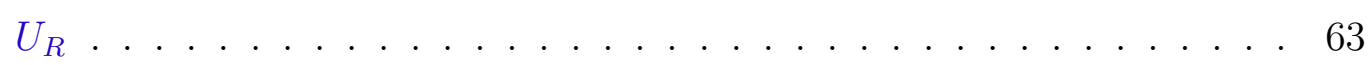

Figura 34 - Mapa dos coeficientes de força para $\delta_{y}=0,10 \ldots \ldots$. . . . . . 64

Figura 35 - Mapa dos coeficientes de força $\overline{C_{x}}$ e $C y_{\text {r.m.s }}$ para o caso de $\delta_{y}=0,10$. 65

Figura 36 - Mapa de Poincaré: VIV Puro . . . . . . . . . . . . . . . . 66

Figura 37 - Mapa de Poincaré: $\delta_{y}=0.10$ e $n_{y}=2 \ldots \ldots$. . . . . . . 66

Figura 38 - Órbitas no espaço de fase caso: VIV Puro . . . . . . . . . . . . . 67

Figura 39 - Órbitas no espaço de fase caso: $\delta_{y}=0.10$ e $n_{y}=2 \ldots \ldots$. . . . . . 67

Figura 40 - Curvas de respostas da amplitude característica na direção cross-wise . 68

Figura 41 - Curvas de respostas da amplitude característica na direção in-line . . . 69

Figura 42 - Series temporais de resposta do cilindro para o caso sem excitação paramétrica . . . . . . . . . . . . . . . . . . . . 69

Figura 43 - Series temporais de resposta do cilindro para o caso $\delta_{y}=\delta_{x}=0,10$ e

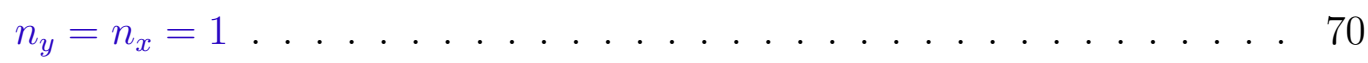

Figura 44 - Curvas de respostas da amplitude característica na direção cross-wise . 71

Figura 45 - Curvas de respostas da amplitude característica na direção in-line . . . 71

Figura 46 - Amplitude característica de oscilação direção na in-line . . . . . . . . . 72

Figura 47 - Series temporais de resposta do cilindro para o caso $\delta_{y}=\delta_{x}=0,10$ e

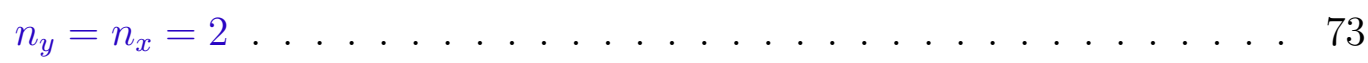

Figura 48 - Series temporais de resposta do cilindro flexível . . . . . . . . . . . 74

Figura 49 - Influência da amplitude de excitação paramétrica nos coeficientes de força em relação à velocidade reduzida $U_{R} \ldots$. . . . . . . . . . 75

Figura 50 - Series temporais de resposta do cilindro utilizando o método das Múltiplas escalas para o caso VIV-1Gl e excitação paramétrica $-U_{R}=5,8$. $\quad 79$ 


\section{Lista de tabelas}

Tabela 1 - Adimensionais propostos por Khalak e Williamson (1999). A notação dos adimensionais está adaptada à nomenclatura seguida na formulação a ser desenvolvida nesta pesquisa. Adaptado de Franzini (2019). . . . . 27

Tabela 2 - Valores dos parâmetros do modelo de Ogink e Metrikine. . . . . . . . . 38

Tabela 3 - Valores dos parâmetros do modelo de Franzini e Bunzel (2018). . . . . 42

Tabela 4 - Parâmetros utilizados no MMS. . . . . . . . . . . . 78 


\section{Lista de abreviaturas e siglas}

DFC Dinâmica de Fluidos Computacional

EPUSP Escola Politécnica da Universidade de São Paulo

LMO Laboratório de Mecânica Offshore

TLP Tension Leg Plataform

HHT Hilbert-Huang Transform

MMS Método das múltiplas escalas

VIV Vibrações induzidas pela emissão de vórtices

VIV-1GL Vibrações induzidas pela emissão de vórtices em cilindro rígido montado em base elástica com um grau de liberdade

VIV-2GL Vibrações induzidas pela emissão de vórtices em cilindro rígido montado em base elástica com dois graus de liberdade 


\title{
Lista de símbolos
}

\author{
$\epsilon_{y} \quad$ Coeficiente de calibração do oscilador de van der Pol na direção cross- \\ wise \\ $\epsilon_{x} \quad$ Coeficiente de calibração do oscilador de van der Pol na direção in-line \\ Sy Taxa de amortecimento estrutural na direção cross-wise \\ $\zeta_{x} \quad$ Taxa de amortecimento estrutural na direção in-line \\ $\nu \quad$ Viscosidade cinemática do fluido \\ $\rho \quad$ Massa específica do fluido \\ $\tau \quad$ Tempo adimensional \\ $\quad \Omega_{y} \quad$ Frequência de variação da rigidez na direção cross-wise \\ $\Omega_{x} \quad$ Frequência de variação da rigidez na direção in-line \\ $\omega_{s} \quad$ Frequência angular de desprendimento de vórtices \\ $\omega_{N, y} \quad$ Frequência natural angular da estrutura na direção cross-wise \\ $\omega_{N, x} \quad$ Frequência natural angular da estrutura na direção in-line \\ $A_{y} \quad$ Coeficiente de calibração do oscilador de van der Pol na direção cross- \\ wise \\ $A_{x} \quad$ Coeficiente de calibração do oscilador de van der Pol na direção in-line \\ $\hat{A}_{y} \quad$ Amplitude de resposta característica de oscilação na direção cross-wise \\ $\hat{A}_{x} \quad$ Amplitude de resposta característica de oscilação na direção in-line \\ $C_{a} \quad$ Coeficiente de massa adicional \\ $C_{D} \quad$ Coeficiente de arrasto \\ $C_{L} \quad$ Coeficiente de sustentação \\ $C_{L}^{0} \quad$ Amplitude do coeficiente de sustentação obtido para cilindro fixo \\ $C_{D}^{0} \quad$ Amplitude do coeficiente de arrasto obtido para cilindro fixo \\ $c_{y} \quad$ Constante de amortecimento estrutural na direção cross-wise
}


$c_{x} \quad$ Constante de amortecimento estrutural na direção in-line

D Diâmetro do cilindro

$F_{D} \quad$ Força de arrasto

$F_{L} \quad$ Força de sustentação

$F_{V Y} \quad$ Força transversal ao escoamento, devido à emissão de vórtices

$F_{Y} \quad$ Projeção do carregamento hidrodinâmico total na direção cross-wise

$F_{X} \quad$ Projeção do carregamento hidrodinâmico total na direção in-line

$\hat{f} \quad$ Frequência de oscilação normalizada

$f_{s} \quad$ Frequência de desprendimento de vórtices

$f_{N, y} \quad$ Frequência natural do cilindro

$f^{*} \quad$ Relação entre as frequência naturais do cilindro na direção in-line e cross-wise

$\Delta k_{y} \quad$ Amplitude de variação da rigidez na direção cross-wise

$\Delta k_{x} \quad$ Amplitude de variação da rigidez na direção in-line

$k_{y} \quad$ Constante de rigidez da estrutura na direção cross-wise

$k_{x} \quad$ Constante de rigidez da estrutura na direção in-line

$\delta_{y} \quad$ Amplitude de excitação paramétrica adimensionalizada na direção crosswise

$\delta_{x} \quad$ Amplitude de excitação paramétrica adimensionalizada na direção inline

$\bar{k} \quad$ Rigidez média por unidade de comprimento

$n_{y} \quad$ Frequência de excitação paramétrica adimensionalizada na direção crosswise

$n_{x} \quad$ Frequência de excitação paramétrica adimensionalizada na direção inline

L Comprimento do cilindro

$m_{s} \quad$ Massa do cilindro

$m_{a} \quad$ Massa adicional potencial 


\begin{tabular}{|c|c|}
\hline$m^{*}$ & Parâmetro de massa reduzida \\
\hline$q_{y}$ & Variável que representa a esteira de vórtices na direção cross-wise \\
\hline$q_{x}$ & Variável que representa a esteira de vórtices na direção in-line \\
\hline$\hat{q_{y}}$ & $\begin{array}{l}\text { Amplitude do ciclo-limite da equação de van der Pol homogênea na } \\
\text { direção cross-wise }\end{array}$ \\
\hline$\hat{q_{x}}$ & $\begin{array}{l}\text { Amplitude do ciclo-limite da equação de van der Pol homogênea na } \\
\text { direção in-line }\end{array}$ \\
\hline$R e$ & Número de Reynolds \\
\hline$S t$ & Número de Strouhal \\
\hline$t$ & Tempo dimensional \\
\hline$U$ & Velocidade do fluido, relativa ao cilindro \\
\hline$U_{\infty}$ & Velocidade ao longe do escoamento incidente \\
\hline$U_{R}$ & Velocidade reduzida \\
\hline$Y$ & Deslocamento transversal do cilindro \\
\hline$X$ & Deslocamento longitudinal do cilindro \\
\hline$y$ & $\begin{array}{l}\text { Deslocamento transversal do cilindro adimensionalizado pelo seu diâme- } \\
\text { tro, } y=Y / D\end{array}$ \\
\hline$x$ & $\begin{array}{l}\text { Deslocamento longitudinal do cilindro adimensionalizado pelo seu diâ- } \\
\text { metro, } x=X / D\end{array}$ \\
\hline$\sigma_{y}$ & Desvio padrão \\
\hline
\end{tabular}




\section{Sumário}

INTRODUÇÃO $\ldots \ldots \ldots \ldots \ldots \ldots \ldots \ldots$

1.1 Objetivos, ganhos esperados e organização do texto $\ldots \ldots \ldots$

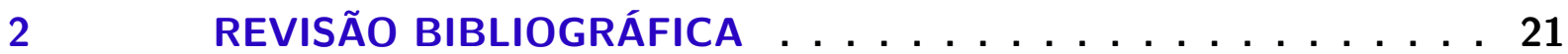

$2.1 \quad$ Escoamento ao redor de um cilindro fixo . . . . . . . . . . . 21

2.2 O fenômeno de VIV em cilindros rígidos montados em base elástica 26

2.3 O fenômeno de VIV em cilindros rígidos montados em base elástica com 2 graus de liberdade . . . . . . . . . . . . . . . . . . 29

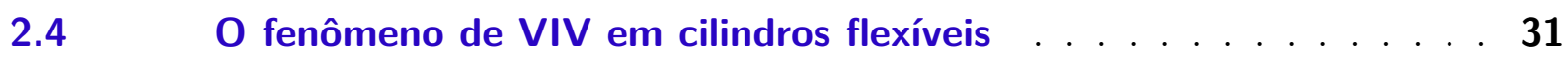

$2.5 \quad$ Modelos fenomenológicos . . . . . . . . . . . . . 33

2.5.1 Modelo fenomenológico de Ogink e Metrikine (2010) . . . . . . . . . 35

2.5.2 Modelo fenomenológico de Franzini e Bunzel (2018) . . . . . . . . . 38

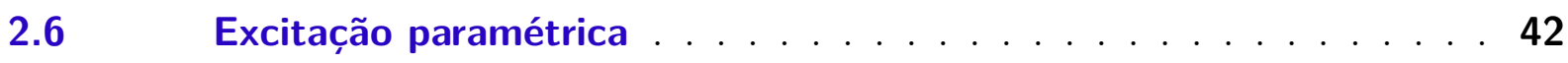

2.7 Problema combinado de excitação paramétrica e VIV . . . . . . . 44

$2.8 \quad$ Lacunas de conhecimento na literatura . . . . . . . . . . . . 45

3 MODELAGEM MATEMÁTICA E METODOLOGIA DE SIMULAÇÃO 46

3.1 Equações governantes para o problema VIV-1GL concomitante com a excitação paramétrica . . . . . . . . . . . . . . 47

3.2 Equações governantes para o modelo VIV-2GL concomitante com a excitação paramétrica . . . . . . . . . . . . . . . . 48

$3.3 \quad$ Metodologia . . . . . . . . . . . . . . . . . 50

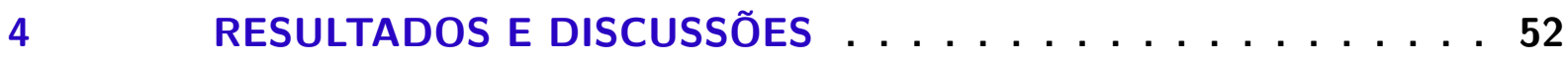

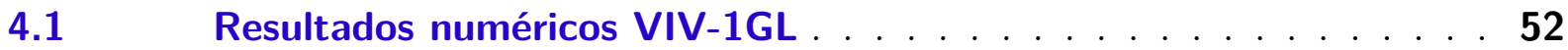

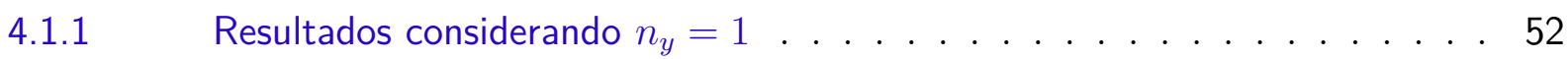

4.1.2 Resultados considerando $n_{y}=2 \ldots \ldots \ldots \ldots$

4.1.3 Coeficientes de força hidrodinâmica VIV-1GL . . . . . . . . . . 63

4.1 .4 Seção de Poincaré . . . . . . . . . . . . . . . . . 66

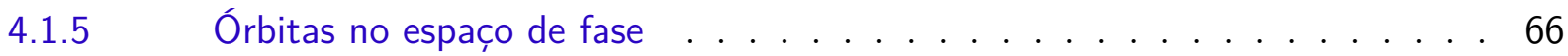

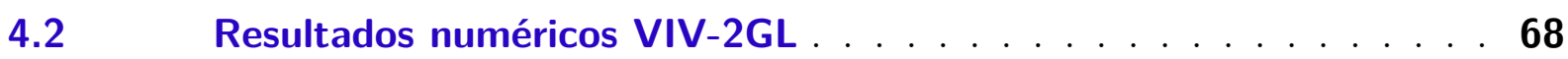

4.2.1 Resultados considerando $n_{y}=n_{x}=1 \ldots \ldots$. . . . . . 68

4.2.2 Resultados considerando $n_{y}=n_{x}=2 \ldots \ldots \ldots \ldots$

4.2.3 Coeficientes de força hidrodinamica VIV-2GL . . . . . . . . . . 73

4.3 Solução assintótica utilizando o Método das Múltiplas Escalas - VIV

$1-\mathrm{GL} \ldots \ldots \ldots \ldots \ldots \ldots \ldots$ 
CONSIDERAÇÕES FINAIS . . . . . . . . . . . . . . 80

Referências . . . . . . . . . . . . . . . . . . 82 


\section{Introdução}

O estudo do comportamento dinâmico das estruturas possui grande importância em diversas áreas das engenharias civil, mecânica, naval, entre outras. Não prever a ocorrência de alguns efeitos dinâmicos pode prejudicar o sistema, pois esses efeitos tendem a elevar os níveis de tensão no material, ou ainda, acelerar a fadiga estrutural.

No campo de estudo de estruturas oceânicas, uma classe de estrutura que possui diversos estudos é a submarina, área que engloba os risers. Essas estruturas são tubulações com grandes comprimentos da ordem de quilômetros suspensos desde a base de produção até o leito, sendo que os principais usos dessas estruturas são fixação, perfuração, produção e injeção. Os risers podem apresentar diversas configurações de posição que variam de acordo com a profundidade e as condições ambientais, sendo definidas como catenária livre, vertical ou lazy-wave, que funcionam como duas catenárias livres divididas por boias submersas.

A tubulação utilizada em uma instalação de exploração de petróleo e gás pode ser classificada como rígida ou flexível. A tubulação rígida é formada por tubos de aço, enquanto que a flexível é construída com diversas camadas de aço, fibras poliméricas e ligas metálicas. Esses tipos de estruturas são submetidos a diversos tipos de carregamentos, tal que nesta pesquisa é dada ênfase aos carregamentos hidrodinâmicos que são responsáveis pelo fenômeno de vibrações induzidas pela emissão de vórtices (VIV).

O VIV é um problema clássico de interação fluido-estrutura em corpos rombudos, como os risers. Consiste em um fenômeno não linear devido ao fato de a excitação causada pela esteira vorticosa afetar a vibração. Além disso, pode-se dizer que o VIV é um fenômeno auto-excitado e autolimitado, sendo as amplitudes máximas na ordem de um diâmetro estrutural.

A motivação para essa pesquisa está no estudo da resposta de risers à excitação simultânea pelo VIV e pela excitação paramétrica. A Figura 1 mostra a representação de uma unidade flutuante sujeita ao movimento de topo e ao carregamento hidrodinâmico causado por correntes marítimas.

Este trabalho está ligado às linhas de pesquisa desenvolvidos no Laboratório de Mecânica Offshore (LMO), grupo responsável pela condução de um abrangente projeto de $\mathrm{P} \& \mathrm{D}$ focado na dinâmica não linear de risers. Esse projeto, apoiado pela Petrobras e conduzido entre 2009 e 2012, contou com atividades em diversas frentes, incluindo campanhas experimentais com modelos flexíveis verticais sob ação simultânea de correnteza e movimento vertical prescrito ao topo. A base de dados experimental gerada é bastante 
Figura 1 - Representação de um modelo esquemático do riser flexível submetido ao VIV e a movimentos de topo.

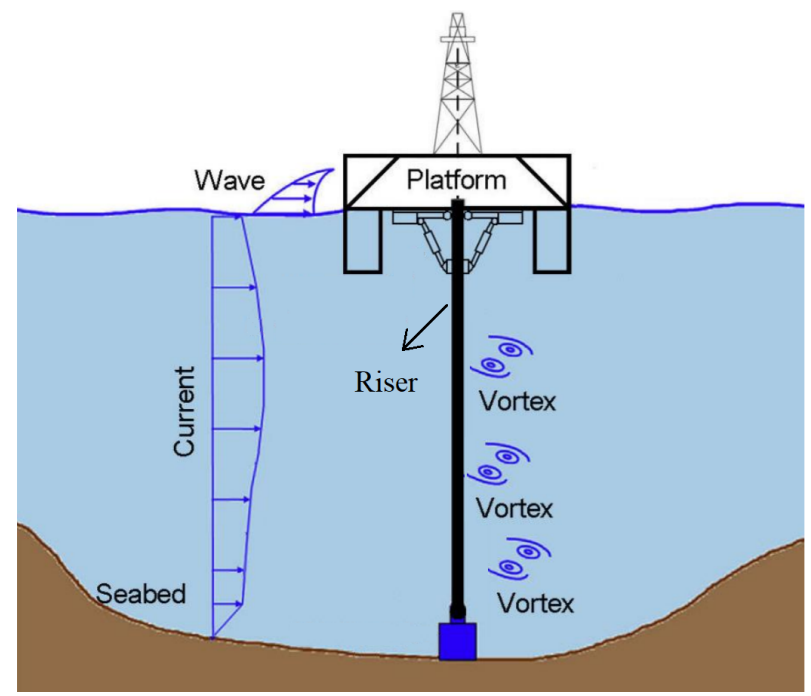

Fonte: Adaptada de Zhang et al. (2020).

inovadora tanto em termos de condições ensaiadas quanto de procedimentos experimentais ${ }^{1}$, e permite a correlação com modelos numéricos, como aqueles empregados nesta dissertação. Embora os resultados experimentais já estejam publicados na forma de artigos científicos (ver Franzini et al. (2015) com cilindro flexível semi imerso e o de Franzini et al. (2018) com cilindro totalmente imerso), na tese de livre-docência de Franzini (2019) e no relatório técnico correspondente (Pesce et al. (2013)), há espaço para novas análises experimentais.

\subsection{Objetivos, ganhos esperados e organização do texto}

O objetivo central desta pesquisa é estudar numericamente o problema de excitação paramétrica concomitantemente com o fenômeno de vibrações induzidas pela emissão de vórtices (VIV) em cilindros rígidos montados em apoio elástico livres para oscilar nas duas direções do plano horizontal. A excitação paramétrica foi modelada na forma de uma variação de rigidez harmônica e monocromática ao redor de um certo valor médio. Já o VIV foi estudado utilizando modelos de ordem reduzida do tipo fenomenológico.

Com este trabalho espera-se compreender quais são as principais características do problema de VIV concomitante com excitação paramétrica em cilindro rígido para toda região de lock-in, fazendo uma análise crítica do que os modelos fenomenológicos conseguem recuperar em um problema mais simples que aquele estudado experimentalmente com cilindro flexível.

1 Ao contrário do que era comum à época (a saber, uso de extensômetros e/ou acelerômetros), os deslocamentos ao longo do cilindro flexível foram medidos de maneira direta por meio de um sistema óptico. 
O texto está dividido em cinco Capítulos. O Capítulo 2 apresenta a revisão bibliográfica, na qual são mostrados os principais conceitos envolvidos nesta dissertação como, o fenômeno de VIV e também o problema de excitação paramétrica. Ao final da revisão bibliográfica discute-se sobre os primeiros trabalhos relacionando os efeitos combinados de VIV com excitação paramétrica e as lacunas de conhecimento. O Capítulo 3 mostra o equacionamento matemático desenvolvido para o problema da excitação paramétrica concomitantemente com o VIV e também apresenta a metodologia numérica utilizada para a análise de resultados. O Capítulo 4 apresenta os resultados e discussões. Por fim, o Capítulo 5 mostra as conclusões a respeito dos resultados obtidos nessa pesquisa e as sugestões para trabalhos futuros. 


\section{Revisão Bibliográfica}

Este Capítulo tem como objetivo apresentar os principais conceitos abordados nesta pesquisa e foi dividido em oito Seções. A Seção 2.1 visa a apresentar os principais conceitos existentes na interação fluido-estrutura do problema clássico de escoamento ao redor de um cilindro fixo. A Seção 2.2 apresenta a conceituação sobre fenômeno de VIV. A Seção 2.3 traz os principais aspectos do fenômeno de VIV em cilindros rígidos montado em apoio elástico com dois graus de liberdade. A Seção 2.4 apresenta uma breve contextualização a respeito de cilindros flexíveis. A Seção 2.5 versa sobre os modelos fenomenológicos e descreve aquele utilizado nesta pesquisa. Feita a descrição do modelo matemático, a Seção 2.6 abrange os principais aspectos sobre a excitação paramétrica. A Seção 2.7 apresenta estudos relacionados ao problema combinado dos fenômenos VIV e excitação paramétrica. Por fim, na Seção 2.8, são identificadas algumas lacunas de conhecimento que poderão ser melhor compreendidas com esta pesquisa.

\subsection{Escoamento ao redor de um cilindro fixo}

O escoamento ao redor de cilindros é um problema clássico na engenharia, pois possui grande complexidade devido à interação entre o fluido e o corpo. Segundo Bearman (1984), cilindros podem ser classificados como corpos rombudos, pois possuem uma porção significativa de sua superfície submersa em fluido submetida ao fenômeno da separação do escoamento. Esse fenômeno ocorre para fluidos reais, ou seja, com viscosidade não nula.

Quando as partículas de fluido atingem o ponto de estagnação, são forçadas a contornar o corpo devido à alta pressão. Contudo, existem forças de atrito que retardam o movimento do fluido até que ocorra a inversão no sentido do escoamento. Isso está ligado diretamente com a separação do escoamento, que provocará o surgimento de duas camadas cisalhantes livres com circulação contrária. Após separadas da superfície do corpo, as camadas cisalhantes livres formam as regiões com alta circulação. Então inicia-se a formação e o desprendimento de vórtices à jusante do cilindro, criando uma região com variação de pressão. A Figura 2 apresenta as regiões do escoamento perturbado pela presença do corpo rombudo.

O modelo proposto por Gerrard (1966) explica a interação entre as camadas cisalhantes no desprendimento de vórtices em um cilindro. Esse modelo é visto na Figura 3, na qual os caminhos das partículas de fluido podem seguir em três direções diferentes: caminho (a) as partículas tendem a se unir ao vórtice que está em formação; a parcela que segue o caminho (b) move-se na direção da camada cisalhante oposta e interrompendo a alimentação de circulação. Finalmente, o caminho (c) auxilia na formação de outro vórtice, 
Figura 2 - Regiões de escoamento perturbado pela presença de um corpo rombudo.

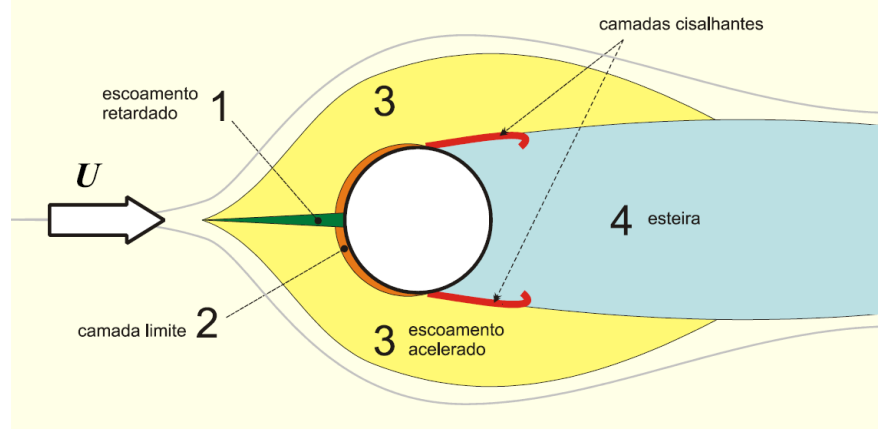

Fonte: Extraído de Assi (2005)

na camada cisalhante inferior, gerando o desprendimento de vórtices.

Figura 3 - Modelo bidimensional de formação e desprendimento de vórtices.

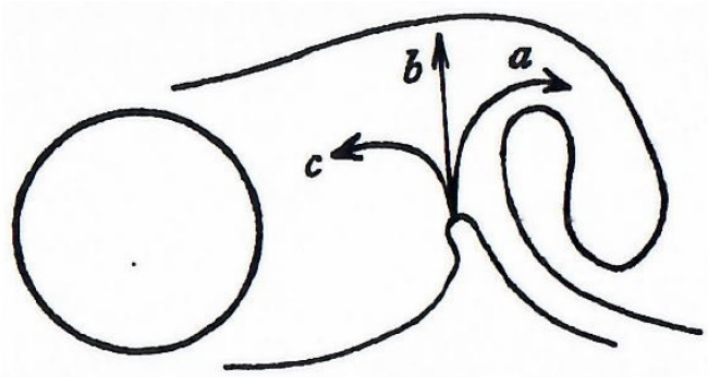

Fonte: Extraído de Gerrard (1966)

Um fenômeno que possui muita importância é o da transição de escoamento ao redor de cilindros passando do regime laminar para o regime turbulento. A transição ocorre devido ao aumento do número de Reynolds (Re), mostrado na Equação 2.1. Conforme o número de Reynolds aumenta, o escoamento vivencia diferentes regimes para cada região de fluido perturbada. A Figura 4 ilustra os diferentes comportamentos do escoamento ao redor de um cilindro em função do número de Reynolds.

$$
R e=\frac{U_{\infty} D}{\nu}
$$

Para $R e<5$, percebe-se que o escoamento é simétrico, no qual as duas camadas cisalhantes permanecem fixas ao corpo do cilindro. No intervalo $5 \leq R e<40$, o escoamento é estável, porém há formação de dois vórtices recirculantes fixos. Após o aumento do número de Reynolds para o intervalo $40 \leq R e<70$, a esteira passa a apresentar sinais de instabilidade, sofrendo oscilações. Inicia-se então, o desprendimento de vórtices alternado, onde é possível notar que a esteira formada a jusante do cilindro possui uma estrutura periódica bem organizada, que é chamada de esteira de Von Kárman. 
Figura 4 - Representação do regime da esteira vorticosa em função do número de Reynolds.
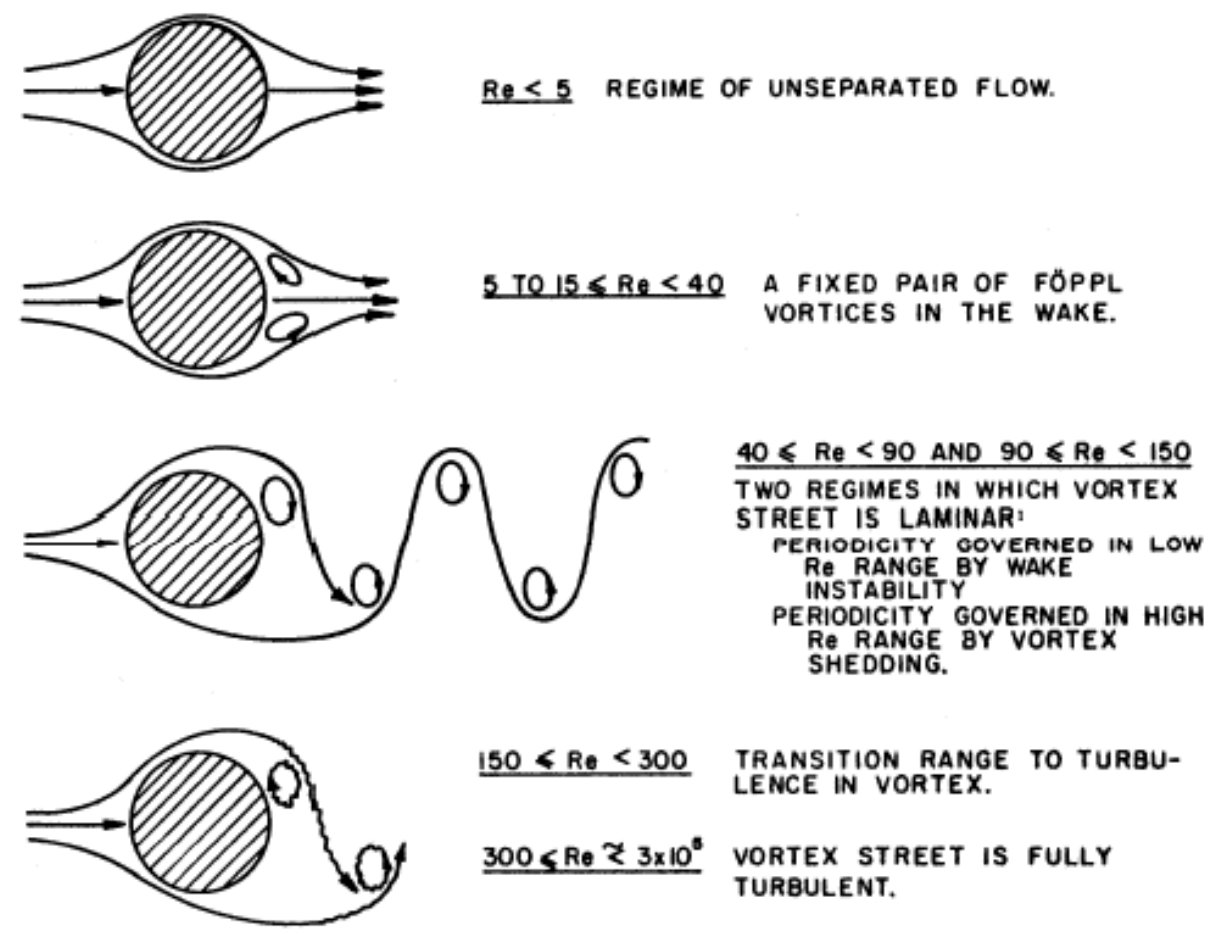

$150<$ Re $<300$ TRANSITION RANGE TO TURBU-
LENCE IN VORTEX.

$300<$ Re $₹ 3 \times 10^{\circ}$ VORTEX STREET IS FULLY

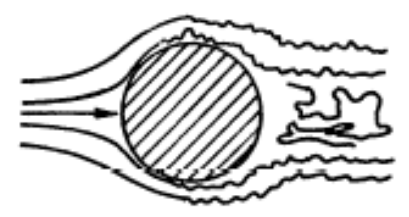

$3 \times 10^{6}<R_{e}<3.5 \times 10^{6}$

LAMINAR BOUNDARY LAYER HAS UNDERGONE

TURBULENT TRANSITION. THE WAKE IS

NARROWER AND DISORGANIZED. NO

VORTFY STRFFT IS $\triangle P P \triangle B F N T$

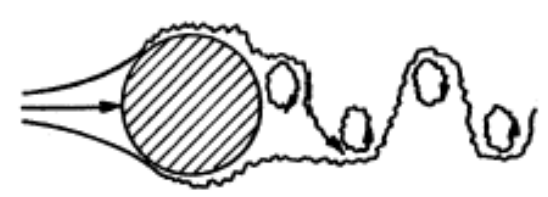

$3.5 \times 10^{\circ}<\operatorname{Re}<\infty$ (?)

RE-ESTABLISHMENT OF THE TURBU-

LENT VORTEX STREET THAT WAS

EVIDENT IN $300<$ Re $₹ 3 \times 10^{\circ}$.

THIS TIME THE BOUNDARY LAYER

IS TURBULENT AND THE WAKE

is THINNER.

Fonte: Extraído de Lienhard (1966).

Com $R e=90$, há o desprendimento de vórtices de modo mais regular devido às camadas cisalhantes se enrolarem. No intervalo de $90 \leq R e<150$ a esteira é dita laminar. Considerando o intervalo $150 \leq R e<300$, existe a transição ao longo das camadas cisalhantes do regime laminar para o turbulento. Para um novo aumento do número de Reynolds, o regime alcançado de $300 \leq R e<3,0 \times 10^{5}$ é denominado subcrítico, em que ocorre a separação do escoamento para um ângulo próximo de $80^{\circ} \mathrm{com}$ relação ao ponto em que ocorre a estagnação frontal. Nesse regime, há transição da camada limite laminar-turbulenta, movendo mais à jusante o ponto no qual ocorre a separação do cilindro e estreitando a esteira de vórtices.

Para o regime crítico, $3,0 \times 10^{5} \leq R e<3,0 \times 10^{6}$, é possível notar diversas mudanças nas características da esteira, pois as camadas cisalhantes tendem a se aproximar da 
superfície do cilindro. Já no regime pós-crítico, $R e \geq 3,0 \times 10^{6}$, a turbulência da camada limite faz aumentar a sua energia cinética, alterando o perfil de velocidade na camada limite.

Devido ao fato de as características da esteira dependerem do número de Reynolds, presume-se uma variação dos coeficientes de arrasto médio $\left(\overline{C_{D}}\right)$ e sustentação $\left(C_{L}\right)$. A frequência de emissão de vórtices $f_{s}$ (do termo em inglês, shedding frequency) depende, essencialmente, da velocidade do escoamento, diâmetro do cilindro e da interação entre as camadas cisalhantes. Ela pode ser representada pelo número de Strouhal (St), apresentado na Equação 2.2.

$$
S t=\frac{f_{s} D}{U_{\infty}}
$$

A Figura 5 apresenta os resultados numéricos e experimentais para a variação do número de Strouhal para uma faixa extensa do número de Reynolds. Pode-se verificar que para ampla faixa de valores do número de Reynolds, o número de Strouhal do escoamento sobre um cilindro fixo é $S t \approx 0,2$.

Figura 5 - Variação do número de Strouhal em função do número de Reynolds.

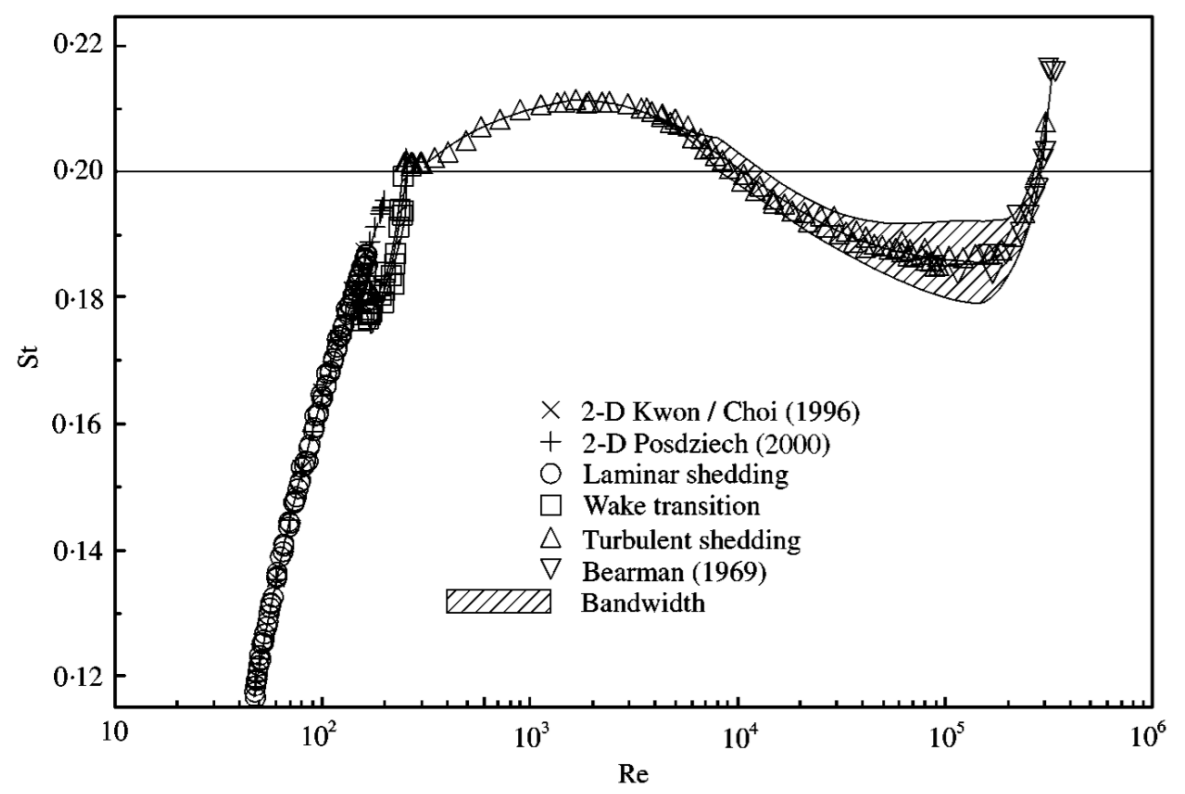

Fonte: Extraído de Norberg (2001)

No estudo de formação de vórtices, existem dois outros parâmetros de fundamental importância que são os coeficientes de força hidrodinâmica agindo sobre o corpo do cilindro. A força pode ser decomposta em duas componentes: força de arrasto $F_{D}$, que é projetada na direção da velocidade relativa entre o cilindro e a correnteza e força de sustentação $F_{L}$, projetada perpendicular à anterior. São definidos os coeficientes relacionados às forças como coeficiente de arrasto $C_{D}$ e sustentação $C_{L}$, apresentados nas Equações 2.3 e 2.4. 


$$
\begin{aligned}
C_{D} & =\frac{2 F_{D}}{\rho U_{\infty}^{2} D L} \\
C_{L} & =\frac{2 F_{L}}{\rho U_{\infty}^{2} D L}
\end{aligned}
$$

sendo $\rho$ a massa específica do fluido e $L$ o comprimento imerso do cilindro.

A variação das forças hidrodinâmicas que atuam no escoamento ao redor de um cilindro estacionário apresentam um caráter cíclico, devido à formação da esteira de vórtices. Por consequência, o campo de tensões de cisalhamento e pressões será variável e os coeficientes de força podem ser escritos como um valor médio $\bar{C}$ e uma outra parcela flutuante $C^{\prime}$, conforme mostrado nas Equações 2.5 e 2.6.

$$
\begin{aligned}
C_{D} & =\overline{C_{D}}+C_{D}^{\prime} \\
C_{L} & =\overline{C_{L}}+C_{L}^{\prime}
\end{aligned}
$$

Quando um vórtice é formado, existe uma diminuição na pressão ao redor do cilindro, o que gera uma força de sustentação no mesmo sentido. Após um ciclo completo de emissão, a força de sustentação passa pelo seu valor máximo (em módulo) e retorna ao valor inicial. Dessa maneira, como são formados dois vórtices para cada ciclo, a força de arrasto se modifica duas vezes, como consequência, a frequência do arrasto é o dobro da frequência de emissão. Além disso, percebe-se que a força na direção transversal é maior que na direção longitudinal, pois o arrasto não sofre alteração em sua direção, já a sustentação é modificada na direção e na intensidade.

A Figura 6 ilustra o comportamento dos coeficientes de arrasto e sustentação para um cilindro fixo, caracterizando o comportamento da esteira para diversos valores do número de Reynolds. Nota-se que, no começo do regime laminar, não há força resultante de sustentação, somente do arrasto. Conforme o número de Reynolds aumenta, para o intervalo $10^{3} \leq R e<10^{5}$ (denominado regime subcrítico), percebe-se que o coeficiente de arrasto se mantém constante próximo a unidade e o coeficiente de sustentação possui sua máxima amplitude de flutuação. Em seguida, há transição para o regime turbulento e ambos os coeficientes diminuem de valor.

Após apresentado como são formados os vórtices e a correspondente influência do número de Reynolds, parte-se para o problema no qual um cilindro rígido é montado em um apoio elástico, o que levará ao fenômeno de VIV, objeto da próxima Seção. 
Figura 6 - Variação dos coeficientes de sustentação e de arrasto com o número de Reynolds.

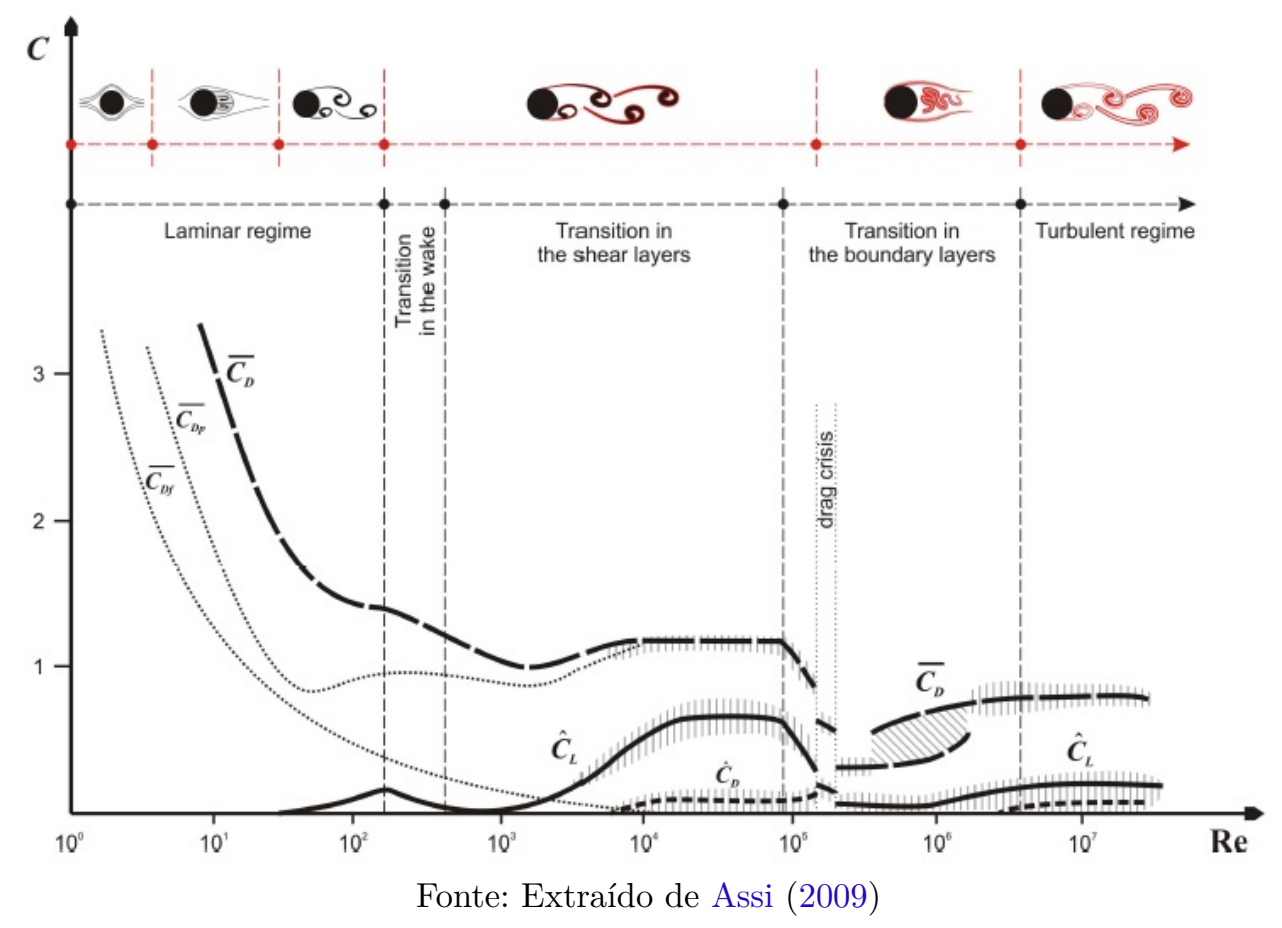

\subsection{O fenômeno de VIV em cilindros rígidos montados em base elástica}

O VIV é um tipo de vibração induzida pelo escoamento observado em corpos rombudos. Como discutido na Seção 2.1, o desprendimento de vórtices ocorre alternadamente, levando a uma variação harmônica das forças envolvidas. O VIV ocorre quando a frequência de excitação $f_{s}$ é próxima da frequência natural do cilindro $f_{N, y}$. Chama-se essa situação de sincronização (do inglês lock-in) e ocorre no intervalo de velocidades reduzidas: $3 \leq U_{R}=\frac{U_{\infty}}{f_{N, y} D} \leq 12$.

Khalak e Williamson (1999) apresentam resultados experimentais de VIV em cilindros rígidos montados em um suporte elástico livre com rigidez $k_{y}$, constante de amortecimento estrutural $c_{y}$ e massa $m_{s}$ livre para oscilar na direção transversal ao escoamento (ou seja, na direção cross-wise). A Figura 7 mostra a representação do modelo abordado neste trabalho. Dentre os trabalhos que estudam esse tema, destacam-se os artigos de Bearman (1984), Sarpkaya (2004), Williamson e Govardhan (2004), Williamson e Govardhan (2008) e Bearman (2011).

Segundo Williamson e Govardhan (2004), o VIV é um fenômeno retroalimentado, pois os efeitos de interação entre o movimento do cilindro e o escoamento interferem na dinâmica da esteira gerada pelo próprio cilindro. Como consequência a movimentação da estrutura fica limitada a uma vibração com amplitude da ordem de um diâmetro. 
Figura 7 - Estudo do fenômeno de VIV em cilindro rígido.

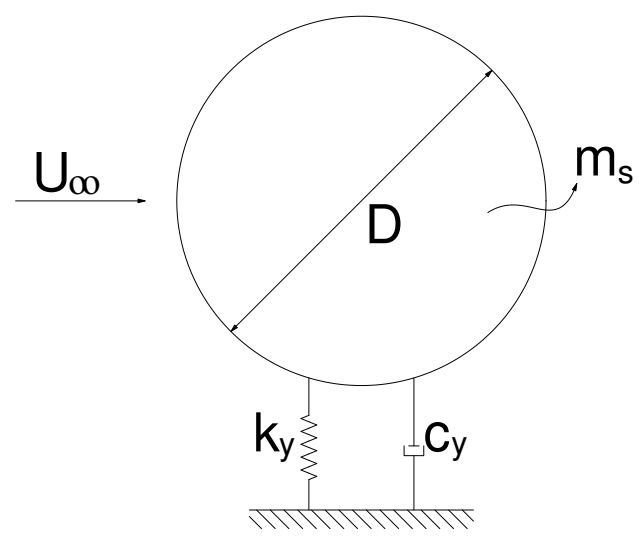

Fonte: O autor

Segundo Khalak e Williamson (1999), os parâmetros adimensionais fornecem uma melhor compreensão para o problema de VIV. Os parâmetros adimensionais de relevo destacados pelos autores são mostrados na Tabela 1.

Tabela 1 - Adimensionais propostos por Khalak e Williamson (1999). A notação dos adimensionais está adaptada à nomenclatura seguida na formulação a ser desenvolvida nesta pesquisa. Adaptado de Franzini (2019).

\begin{tabular}{ccc}
\hline Grandeza & Símbolo & Expressão \\
\hline Amplitude adimensional & $\hat{A}$ & $\frac{\hat{A}_{y}}{D}$ \\
Taxa de amortecimento & $\zeta_{y}$ & $\frac{c_{y}}{2 \sqrt{k_{y}\left(m_{s}+m_{a}\right)}}$ \\
Coeficiente de arrasto & $C_{D}$ & $\frac{2 F_{D}}{\rho U_{\infty}^{2} D L}$ \\
Coeficiente de sustentação & $C_{L}$ & $\frac{2 F_{L}}{\rho U_{\infty}^{2} D L}$ \\
Frequência natural angular & $\omega_{N, y}$ & $2 \pi f_{N, y}$ \\
Frequência adimensional & $\hat{f}$ & $\frac{f}{f_{N, y}}$ \\
Número de Reynolds & $R e$ & $\frac{U_{\infty} D}{\nu}$ \\
Parâmetro de massa reduzida & $m^{*}$ & $\frac{4 m_{s}}{\rho \pi D^{2} L}$ \\
\hline
\end{tabular}

Deve-se dar a devida atenção aos parâmetros adimensionais, de acordo com o meio fluido em que o sistema está submerso, pois existe diferença no comportamento da frequência adimensional entre cilindros ensaiados em ar e na água que é atribuída influência da massa adicional. Dado que o cilindro oscilando no ar a massa de fluido deslocado junto 
com ele é desprezada se comparada à massa do sistema. Porém com a oscilação ocorrendo em água, a massa adicional potencial $m_{a}$, que representa a razão entre a força inercial associado ao fluido e a aceleração do corpo não pode ser desprezada. Vale ressaltar que como o corpo é um cilindro, e assumindo que o corpo esta em imerso um domínio de fluido infinito, mostrou-se que o valor do coeficiente de massa adicional é $C_{a}=1,0$.

Segundo Khalak e Williamson (1999), a massa adicional efetiva mostrada na Equação 2.7, considera o efeito aparente da força transversal total do fluido em fase com a aceleração do corpo por meio do termo $C_{L} \cos (\phi)$, sendo $\phi$ o ângulo de fase entre a força de sustentação e o deslocamento transversal do corpo.

$$
C_{a, e}=\frac{1}{2 \pi^{3}} \frac{C_{L} \cos (\phi)}{A_{y}}\left(\frac{U_{R}}{\hat{f}}\right)^{2}
$$

Para melhor compreensão dos resultados de VIV, são realizadas análises com as curvas de amplitude característica e frequência adimensionais em função da velocidade reduzida. Outro parâmetro que possui suma importância nos resultados é o de massa reduzida $m^{*}$, discutido em diversas referências da literatura como Khalak e Williamson (1999).

A Figura 8 apresenta como a amplitude de resposta do VIV é alterada de acordo com a combinação $m^{*} \zeta_{y}$ (parâmetro de massa-amortecimento) pois, quanto menor seu valor, maior será a amplitude de vibração observada. É possível notar no trabalho de Feng (1968) que a combinação $\left(m^{*} \zeta_{y}\right)$ possui valor mais elevado, já que o autor menciona $m^{*}=248$, enquanto Khalak e Williamson (1999) propuseram $m^{*}=10,1$, lembrando que o experimento de Feng (1968) foi conduzido em túnel de vento e o de Khalak e Williamson (1999) foi realizado em água.

Percebe-se que, para valores baixos do parâmetro $m^{*}$, o sistema apresenta três ramos de resposta distintos, chamados de initial branch, upper branch e o lower branch. $\mathrm{O}$ primeiro ramo, initial branch, é caracterizado por baixas amplitudes com valor máximo na ordem de $\hat{A_{y}} \approx 0,4$ e ocorre no intervalo de velocidades reduzidas $3 \leq U_{R}<4$. O segundo, upper branch, possui amplitude máxima de $\hat{A}_{y} \approx 0,9$ que mantém-se para $4 \leq U_{R}<7$. Nesse ramo há um salto na amplitude devido à sincronização. O último ramo, lower branch, acontece durante o intervalo de $7 \leq U_{R}<11$ e possui amplitude de oscilação constante $\hat{A}_{y} \approx 0,6$. Após o termino do lower branch ocorre a dessincronização e, com isso, a diminuição da amplitude de oscilação.

Outro fato importante que deve-se observar na curva de amplitude da Figura 8 são as mudanças entre os ramos, onde notam-se saltos na amplitude de resposta. Conforme visto nos trabalhos de Williamson e Roshko (1988) e Khalak e Williamson (1999), as duas transições estão relacionadas com os diferentes padrões de emissão de vórtices em cada ramo. 
Figura 8 - Curva de amplitude de resposta em função da velocidade reduzida em cilindro rígido.

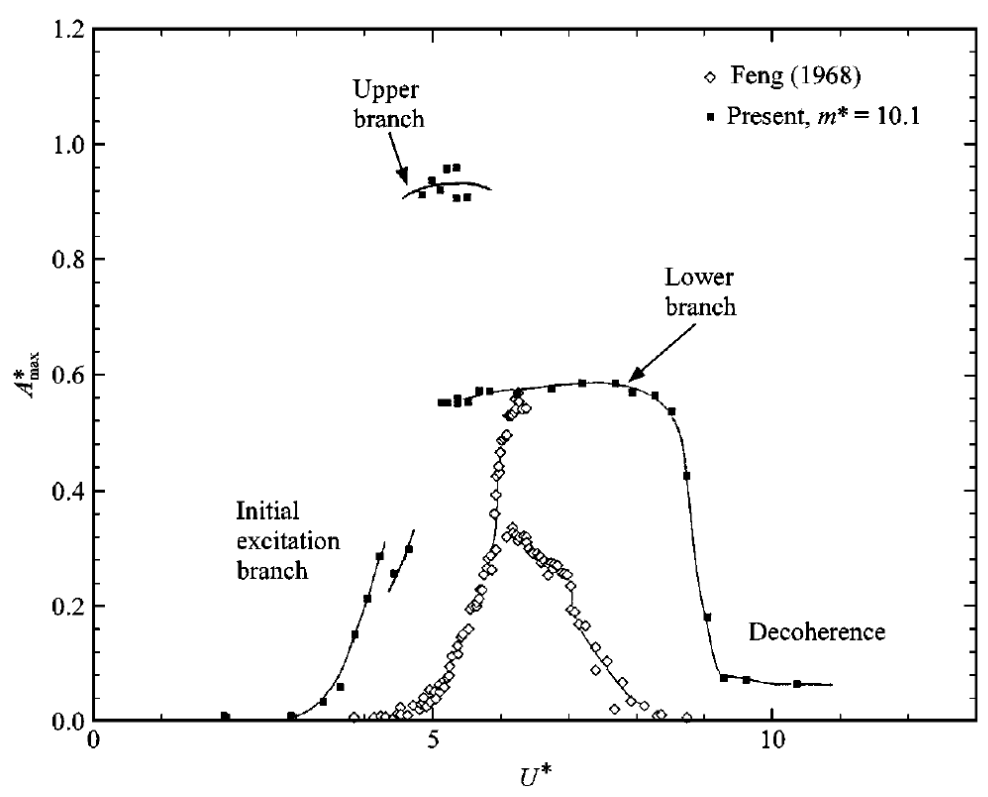

Fonte: Extraído de Khalak e Williamson (1999).

No trabalho de Williamson e Govardhan (2004), foram mapeados os padrões de emissão de vórtices para um vasto intervalo de velocidades reduzidas que é mostrado na Figura 9 ilustra essa variação na emissão dos vórtices. No initial branch, para cada ciclo de oscilação são emitidos dois vórtices simples, configurando o padrão "2S" de esteira. O upper branch e o lower branch possuem o mesmo padrão de emissão, que é o "2P", sendo que para cada ciclo formam-se dois pares de vórtices.

Na Figura 10 é possível observar a variação da frequência adimensional em função da velocidade reduzida. Em valores com o parâmetro $m^{*}$ abaixo de 10, nota-se que a frequência adimensional assume valores diferentes de 1,0. Williamson e Govardhan (2008) mostram a influência da massa reduzida $m^{*}$ para valores acima de 10 , onde a frequência de oscilação é aproximadamente igual a frequência natural em água $\hat{f} \approx 1$.

\subsection{O fenômeno de VIV em cilindros rígidos montados em base elástica com 2 graus de liberdade}

O problema de VIV com um grau de liberdade (VIV-1GL) foi estudado ao longo de muito tempo pela comunidade cientifica. Porém nos últimos anos com a motivação da industria offshore, o fenômeno de VIV com liberdade para oscilar nas duas direções transversal e paralelo ao escoamento (VIV-2GL) começou a ser mais explorado.

No trabalho de Jauvtis e Williamson (2004), os autores realizaram o estudo experimental envolvendo o VIV-2GL, para sistemas com o parâmetro $m^{*}$ baixos $\left(m^{*}<6\right)$ 
Figura 9 - Padrões de emissão de vórtices para cada ramo de resposta.

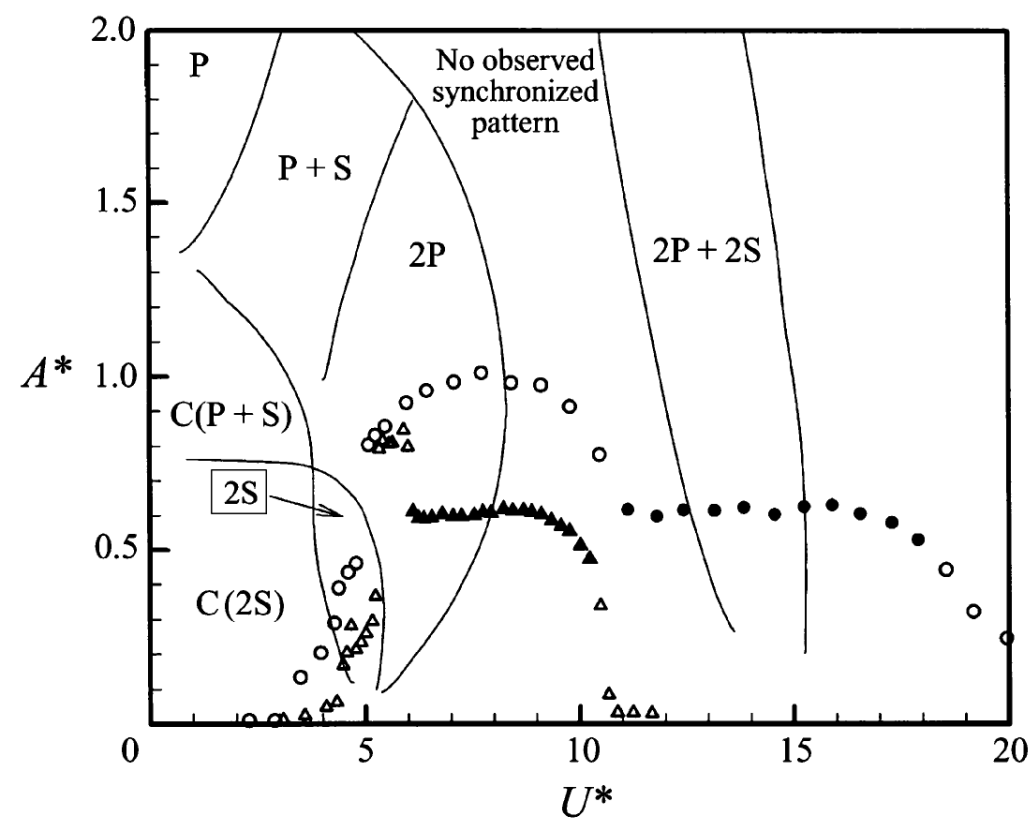

Fonte: Extraído de Williamson e Govardhan (2004)

Figura 10 - Frequência adimensional de oscilação como função da velocidade reduzida.

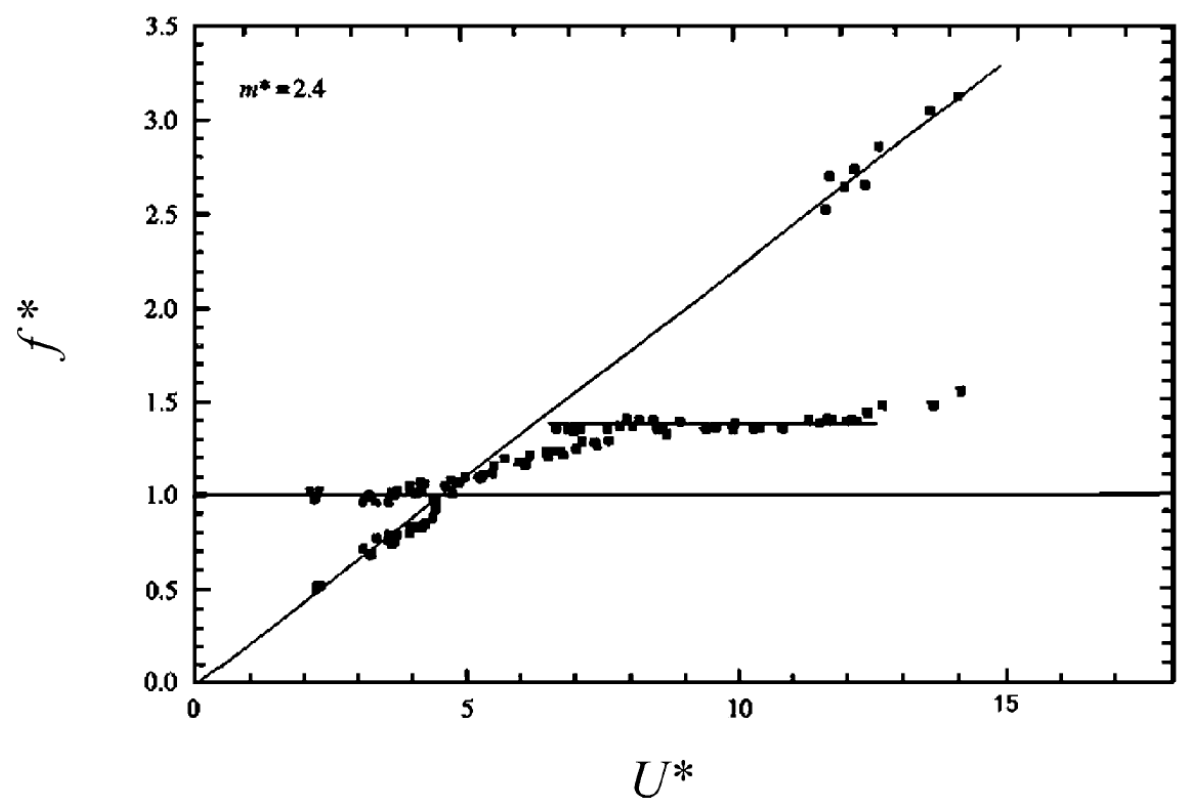

Fonte: Extraído de Govardhan e Williamson (2000).

e para valores moderados $\left(m^{*}>6\right)$. E notaram que para os sistemas com $m^{*}>6$ os resultados obtidos eram semelhantes os encontrados no experimento com VIV-1GL, ou seja, as oscilações in-line não influenciaram a resposta do sistema. Por outro lado os sistemas cujo $m^{*}<6$, os autores observaram que a interação fluido-estrutura foi modificada drasticamente. 
Na Figura 11 é possível observar os resultados experimentais com um cilindro rígido montado em base elástica e sujeito ao VIV-2GL. Essa figura mostra a curva de amplitude de resposta nas direções transversal e paralela ao escoamento, junto com a curva de frequência adimensional de oscilação na direção cross-wise em função da velocidade reduzida.

Para sistemas com baixo parâmetro de massa reduzida, surgiu um novo ramo de amplitude que foi denominado de super-upper branch, onde a amplitude característica na direção cross-wise aumentou consideravelmente para $\hat{A}_{y}=1,5$, enquanto que na direção in-line a amplitude característica foi de $\hat{A}_{x}=0,35$. Essa mudança no pico de amplitude máxima também está associada ao deslocamento do intervalo de lock-in de $U_{R} \approx 5,5$ no VIV-1GL para $U_{R} \approx 8,0$ no VIV-2GL. Além disso, os autores descobriram um novo padrão de emissão de vórtices denominado " $2 \mathrm{~T}$ ", onde são emitidos duas trincas de vórtices por ciclo de oscilação do cilindro, fato diretamente ligado ao novo ramo de resposta.

Blevins e Coughran (2009) investigaram a variação da massa, o amortecimento e o número de Reynolds para as condições VIV-1GL e VIV-2GL. Os autores observaram que devido ao acoplamento das respostas em ambas direções, é gerado um aumento de energia no sistema. Assim como Jauvtis e Williamson (2004), Blevins e Coughran (2009) conseguiram recuperar no plano de fase horizontal $(\mathrm{x}-\mathrm{y})$ trajetórias no formato clássico de "8".

\subsection{O fenômeno de VIV em cilindros flexíveis}

O estudo de risers flexíveis foi acelerado devido a inovações caracterizadas na utilização da exploração de petróleo e gás em águas profundas. Conforme foram se desenvolvendo novas tecnologias para a construção deste tipo de estrutura, também foi preciso desenvolver estudos quanto ao comportamento mecânico e hidrodinâmico (devido aos efeitos de VIV), com intuito de mitigar os efeitos relacionados à fadiga estrutural.

Os cilindros flexíveis possuem uma maior representatividade das estruturas reais, devido a capacidade de abranger efeitos tridimensionais relacionados ao escoamento. Nos cilindros flexíveis, presume-se que os efeitos de VIV alterem as oscilações ao longo do comprimento dos cabos. Um trabalho importante no que diz respeito ao comportamento dinâmico de cilindros flexíveis é Pesce e Fujarra (2000), onde os autores estudaram o comportamento de um cilindro flexível em balanço. Foi visto que o modelo estudado pelos autores apresentou um salto entre os ramos upper-branch e lower-branch para velocidades reduzidas próximas a 8,3 .

Fujarra et al. (2001) investigaram o comportamento de um cilindro flexível submetido ao fenômeno de VIV. O modelo proposto pelos autores apresentou um novo ramo de resposta denominado high-speed-mode para velocidades reduzidas mais elevadas $\left(U_{R}>12\right)$. 
Figura 11 - Curva de amplitude de resposta e frequência adimensional de oscilação em função da velocidade reduzida. VIV-2GL.
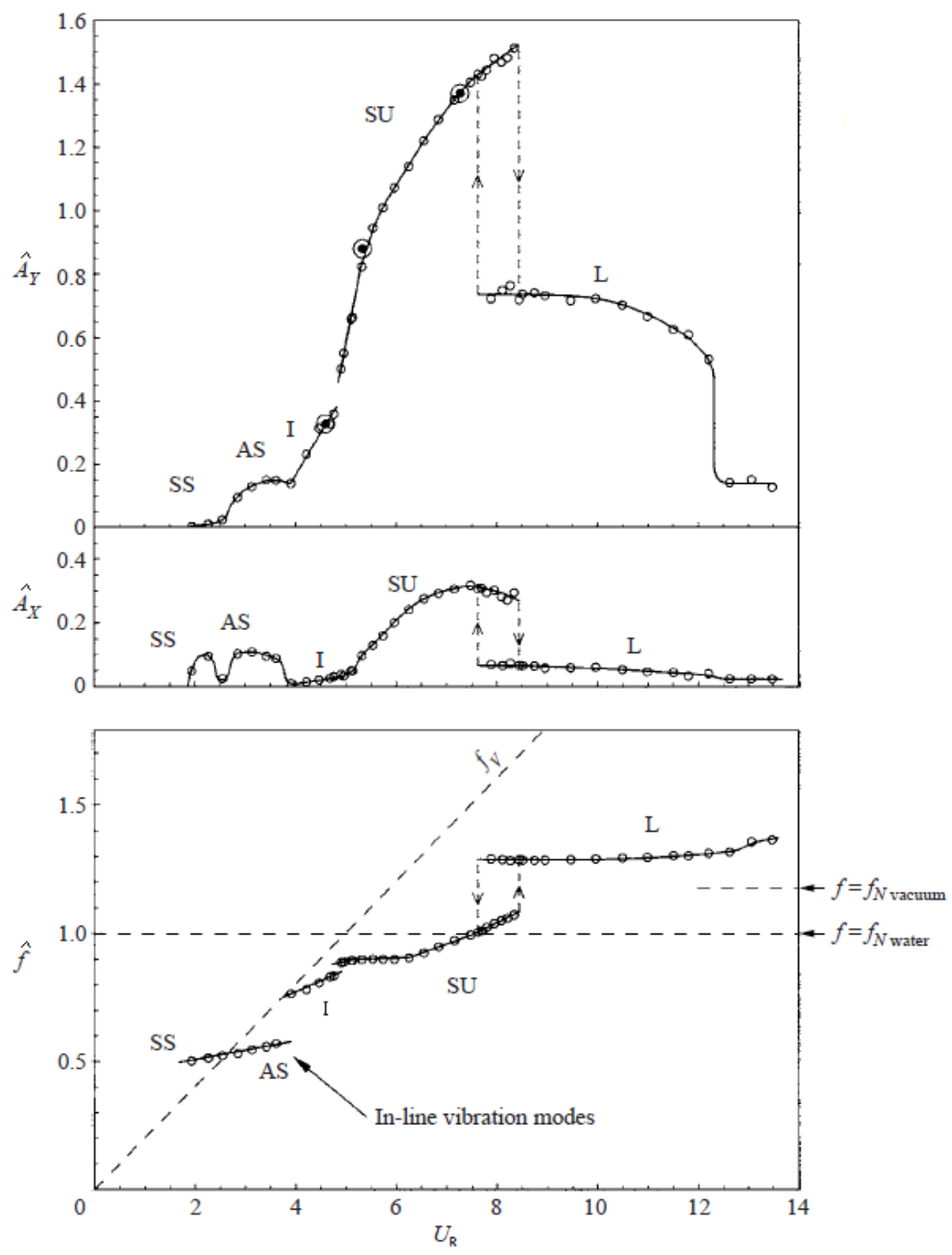

Fonte: Adaptada de Williamson e Govardhan (2004)

Segundo os autores uma possível causa desse ramo está relacionado com a influência mutua das vibrações in-line e cross-wise, além do modelo possuir a frequência natural in-line maior que a frequência natural cross-wise.

O trabalho de Pesce e Fujarra (2005) compara a curva de amplitude de resposta do modelo flexível visto em Pesce e Fujarra (2000) com o modelo de cilindro rígido VIV-2GL estudado por Jauvtis e Williamson (2004), conforme visto na Figura 12. Comparando ambos nota-se que os resultados apresentam certa semelhança, isso ocorre devido a proximidade dos valores do parâmetro $m^{*} \zeta_{y}$.

Segundo Wu, Ge e Hong (2012), o comportamento multi-modal dos cilindros flexíveis longos é a principal diferença para o modelo de cilindro rígido com um grau de 
Figura 12 - Curva de amplitude de resposta em função da velocidade reduzia. Comparação entre a resposta de um cilindro flexível $(* ;+)$ e cilindro rígido com dois graus de liberdade (o).

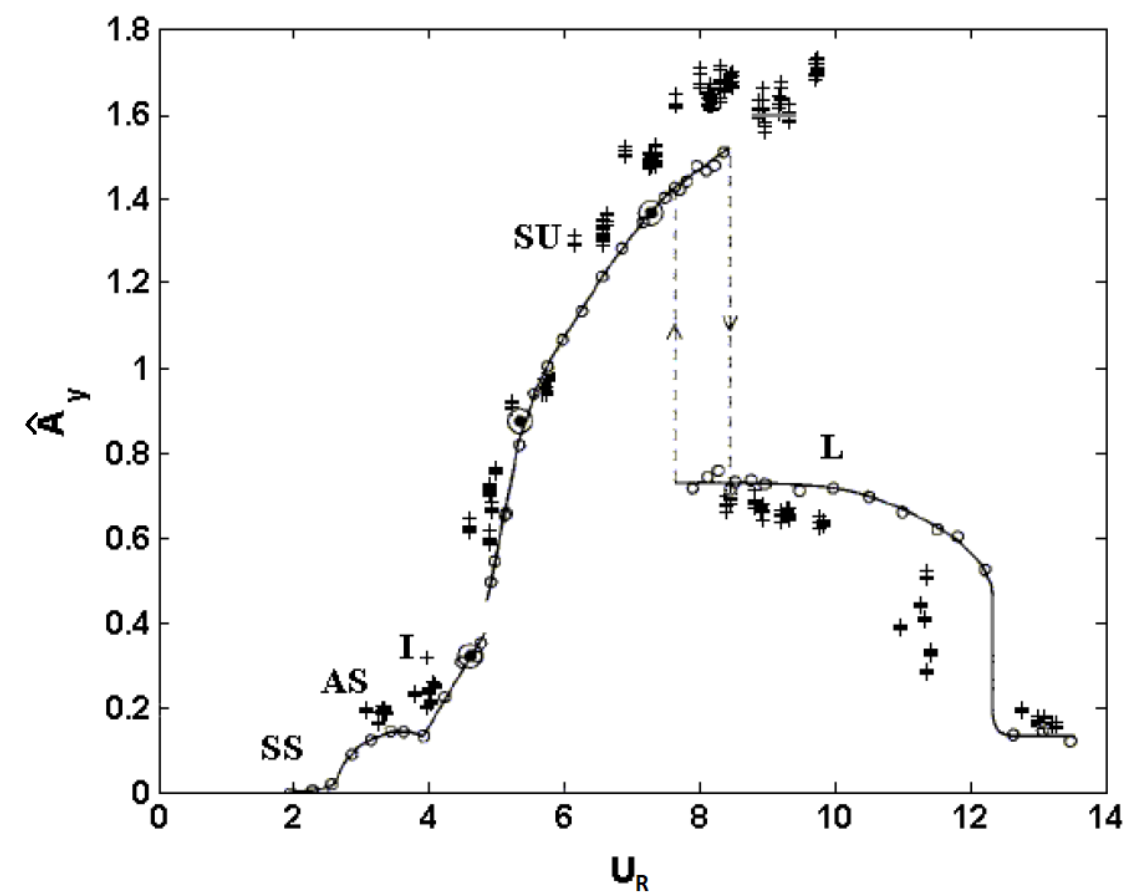

Fonte: Adaptado de Pesce e Fujarra (2005).

liberdade, caracterizado por um único modo de vibrar. Os autores ressaltam que a resposta multi-modal pode ser considerada como uma superposição de vários modos individuais, cada modo corresponde a uma forma de onda estacionária com frequências distintas.

O leitor interessado em aprofundar seus conhecimentos em cilindros flexíveis sob ação do VIV pode consultar o trabalho de Liu et al. (2020), onde os autores fazem uma revisão de literatura a respeito do assunto. Vistos os principais aspectos a respeito do fenômeno de VIV, a Seção seguinte apresenta o modelo matemático de ordem reduzida que representa o VIV.

\subsection{Modelos fenomenológicos}

Nesta Seção são abordados os principais conceitos sobre os modelos matemáticos de ordem reduzida do fenômeno de VIV. Existem três abordagens complementares para estudar esse fenômeno, a saber, experimentos com modelos físicos, simulações numéricas usando modelos hierárquicos de alta ordem baseados em dinâmica dos fluidos computacional (DFC) e investigações usando modelos semi-empíricos baseados no conceito de modelos fenomenológicos.

Os modelos fenomenológicos possuem um custo computacional baixo, comparado 
a uma análise via DFC e que resolve numericamente as equações de Navier-Stokes para o problema de interação fluido-estrutura. Nos modelos fenomenológicos, as equações dos osciladores não lineares têm origem nas equações de van der Pol ou Rayleigh que descrevem o carregamento do fluido, que por sua vez estão acopladas a um oscilador estrutural através de coeficientes calibrados experimentalmente.

Os primeiros trabalhos nesta área são os de Birkhoff (1953), que tentou encontrar expressões que correlacionassem o número de Strouhal e o espaçamento dos vórtices da esteira por meio de um oscilador linear. Bishop e Hassan (1964) mostraram que as forças que atuam no cilindro podem ser modeladas como solução de um oscilador auto-excitado e não linear. Dentre os diversos trabalhos publicados que utilizam osciladores não lineares para modelar a força transversal, deve-se destacar Skop e Griffin (1973) e Iwan e Blevins (1974) que utilizam a equação de van der Pol para descrever a dinâmica da esteira.

Cunha (2005) faz uma análise crítica dos resultados apresentados por diferentes modelos fenomenológicos até aquele momento. Segundo o autor, os modelos podem ser promissores desde que incorporem informações bem definidas a respeito das bifurcações que ocorrem devido a mudança no padrão de emissão de vórtices, especialmente entre os ramos upper e lower branches.

O trabalho desenvolvido por Poncet (2011) envolveu a criação de um modelo analítico para prever as oscilações de um riser rígido, utilizando o modelo de Iwan e Blevins (1974) para modelar o carregamento hidrodinâmico devido ao VIV. O autor aplicou o método das múltiplas escalas para resolução analítica e obter as amplitudes adimensionais do riser.

Facchinetti, de Langre e Biolley (2004) buscam o acoplamento entre a equação de van der Pol e o oscilador estrutural, por meio de um termo forçante que é proporcional a aceleração do cilindro. Adotaram, ainda, como hipótese que a velocidade de translação do cilindro é muito menor do que a velocidade da correnteza $\left(\frac{d Y}{d t} \ll U_{\infty}\right)$. Além disso, os autores sugerem calibração única para ambos ramos de resposta (upper-branch e lower-branch). No entanto, em Ogink e Metrikine (2010), os autores sugerem uma calibração diferente para cada ramo de resposta, com o intuito de aprimorar a aderência com os resultados experimentais. Esse último modelo fenomenológico é discutido com mais profundidade na Subseção 2.5.1.

Os modelos fenomenológicos para o caso VIV-2GL, são de grande interesse da comunidade científica. Pois a conforme visto na Seção 2.3, a presença das vibrações in-line pode interferir na resposta do sistema, já que o padrão da esteira é alterado e consequentemente a resposta na direção cross-wise também. Para o leitor que deseje mais detalhes sobre esses modelos, pode-se consultar os trabalhos de Srinil e Zanganeh (2012), Postnikov, Pavlovskaia e Wiercigroch (2017) e Qu e Metrikine (2020). 
O presente trabalho utiliza o modelo fenomenológico proposto por Franzini e Bunzel (2018) para descrever o carregamento hidrodinâmico resultante do VIV-2GL. A Subseção 2.5.2 apresenta o modelo detalhadamente.

\subsubsection{Modelo fenomenológico de Ogink e Metrikine (2010)}

Esta Subseção apresenta o modelo fenomenológico de VIV para cilindro rígidos montados em base elástica proposto por Ogink e Metrikine (2010).

O modelo de Ogink e Metrikine (2010) descreve tanto os experimentos com oscilação livre quanto a forçada, contemplando a descrição correta para o intervalo de velocidades reduzidas na região de lock-in e a amplitude de resposta do cilindro.

De acordo com o que foi observado na Seção 2.2, a interação do fluido com o cilindro altera o comportamento da esteira, ou seja, altera também as forças hidrodinâmicas. A Equação 2.8 representa uma forma de modelar o movimento da esteira de vórices, utilizando o oscilador não linear de van der Pol.

$$
\frac{d^{2} q_{y}}{d t^{2}}+\epsilon_{y} \omega_{s}\left(q_{y}^{2}-1\right) \frac{d q_{y}}{d t}+\omega_{s}^{2} q_{y}=0
$$

O uso da equação de van der Pol se dá devido a particularidades presentes entre o processo de formação da esteira vorticosa e auto-limitação e auto-excitação dos osciladores não lineares. A variável proveniente da esteira $q_{y}$, pode ser vista na Equação 2.8, no qual $\omega_{s}$ é a frequência de emissão de vórtices. Assim, essa equação pode ser empregada para representar o coeficiente de sustentação agindo sobre um cilindro fixo.

Dessa forma, utilizando a amplitude de resposta em regime permanente da Equação 2.8 que é $\hat{q}_{y}=2$, pode-se caracterizar o coeficiente de sustentação agindo em um cilindro fixo por meio da Equação 2.9.

$$
C_{L}=\frac{q_{y}}{\hat{q_{y}}} C_{L}^{0}
$$

sendo $C_{L}$ o coeficiente de sustentação e $C_{L}^{0}$ a amplitude do coeficiente de sustentação obtido experimentalmente para cilindros fixos sujeitos a emissão de vórtices.

A descrição do modelo começa com a apresentação das equações de movimento na forma dimensional, por meio das Equações 2.10 e 2.11 .

$$
\begin{array}{r}
m_{s} \frac{d^{2} Y}{d t^{2}}+c_{y} \frac{d Y}{d t}+k_{y} Y=F_{Y} \\
\frac{d^{2} q_{y}}{d t^{2}}+\epsilon_{y} \omega_{s}\left(q_{y}^{2}-1\right) \frac{d q_{y}}{d t}+\omega_{s}^{2} q_{y}=f
\end{array}
$$


A formulação proposta no modelo tem como variável principal $q_{y}$ que descreve a interação entre o fluido e o cilindro, $\epsilon_{y}$ é um coeficiente de calibração obtido experimentalmente e $f$ é o termo forçante que acopla o movimento da esteira com o cilindro.

No lado direito da Equação 2.10, $F_{Y}$ é o carregamento transversal hidrodinâmico. Essa força é uma superposição de efeitos entre a força devido à emissão de vórtices $F_{V Y}$ e a força de inércia $F_{P O T}$ associada ao termo de massa adicional como mostra a Equação 2.12 .

$$
F_{Y}=F_{V Y}+F_{P O T}=\frac{1}{2} \rho D L U_{\infty}^{2} C_{V Y}-m_{a} \frac{d^{2} Y}{d t}
$$

O problema pode ser escrito na forma adimensional, considerando os adimensionais listados na Equação 2.13.

$$
\begin{gathered}
y=\frac{Y}{D} ; \quad m^{*}=4 \frac{m_{s}}{\rho \pi D^{2} L} ; \quad \zeta_{y}=\frac{c_{y}}{2\left(m_{s}+m_{a}\right) \omega_{N, y}} ; \quad \tau=\omega_{N, y} t \\
S t=\frac{\omega_{s} D}{2 \pi U_{\infty}} ; \quad U_{R}=\frac{U_{\infty}}{f_{N, y} D} ; \quad C_{a}=4 \frac{m_{a}}{\rho \pi D^{2} L}
\end{gathered}
$$

Deve-se lembrar que a frequência natural é um dimensional definido pela equação 2.14 e a notação de derivação utilizada é dada pela equação 2.15 .

$$
\begin{array}{r}
\omega_{N, y}=\sqrt{\frac{k_{y}}{\left(m_{s}+m_{a}\right)}} \\
\frac{d}{d t}()=\frac{d}{d \tau}() \omega_{N, y}=\omega_{N, y}\left({ }^{\cdot}\right)
\end{array}
$$

Substituindo os adimensionais definidos em 2.13 junto as Equações 2.14 e 2.15 nas Equações 2.10 e 2.11. Obtém-se as equações de movimento adimensionais:

$$
\begin{array}{r}
\ddot{y}+2 \zeta_{y} \dot{y}+y=\frac{U_{R}^{2}}{2 \pi^{3}\left(C_{a}+m^{*}\right)} C_{V Y} \\
\ddot{q}_{y}+\epsilon_{y} S t U_{R}\left(q_{y}^{2}-1\right) \dot{q}_{y}+\left(S t U_{R}\right)^{2} q_{y}=A_{y} \ddot{y}
\end{array}
$$

O coeficiente $C_{V Y}$ representa a parcela de força transversal que depende da velocidade transversal e do ângulo de ataque $(\beta)$. Na Figura 13 é possível observar a representação da decomposição de forças, a força de arrasto é definida como paralela à velocidade relativa de incidência do fluido $U=\sqrt{U_{\infty}^{2}+\left(\frac{d Y}{d t}\right)^{2}}$, enquanto a força de sustentação é perpendicular à direção de $U$.

Dessa forma, é possível escrever as Equações 2.18 e 2.19. 
Figura 13 - Representação da dedução de $C_{V Y}$. O deslocamento do cilindro é adotado para baixo.

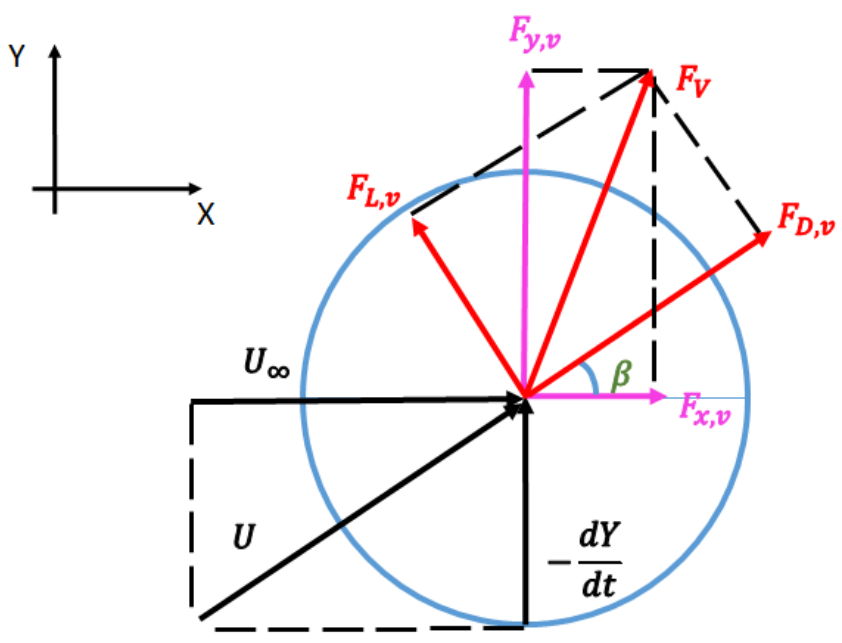

Fonte: O autor.

$$
\begin{gathered}
F_{V Y}=\frac{1}{2} \rho D L U_{\infty}^{2} C_{V Y}=F_{L, v} \cos \beta+F_{D, v} \operatorname{sen} \beta \\
C_{V Y}=\left(\frac{U}{U_{\infty}} C_{L}-\frac{U}{U_{\infty}^{2}} \frac{d Y}{d t} C_{D}\right)
\end{gathered}
$$

onde $\beta$ é o ângulo entre a direção da velocidade relativa do fluido $U$ e a velocidade incidente $U_{\infty}$. Assim, pode-se fazer as seguintes relações trigonométricas como mostram as Equações 2.20, 2.21, 2.22 e 2.23.

$$
\begin{array}{r}
\beta=-\arctan \left(\frac{\frac{d Y}{d t}}{U_{\infty}}\right) \\
\operatorname{sen} \beta=-\frac{1}{U} \frac{d Y}{d t}=-\frac{2 \pi \dot{y}}{U_{R} \sqrt{1+\left(\frac{2 \pi \dot{y}}{U_{R}}\right)^{2}}} \\
\cos \beta=\frac{U_{\infty}}{U}=\frac{1}{\sqrt{1+\left(\frac{2 \pi \dot{y}}{U_{R}}\right)^{2}}} \\
U=\sqrt{U_{\infty}+\left(\frac{d Y}{d t}\right)^{2}}=U_{\infty} \sqrt{1+\left(\frac{2 \pi \dot{y}}{U_{R}}\right)^{2}}
\end{array}
$$

Reescrevendo o coeficiente $C_{V Y}$ em sua forma adimensional, têm-se:

$$
C_{V Y}=\left(C_{L}-\frac{C_{D}}{U_{R}} 2 \pi \dot{y}\right) \sqrt{1+\left(\frac{2 \pi \dot{y}}{U_{R}}\right)^{2}}
$$


Sendo assim, é possível descrever o fenômeno do VIV de um cilindro rígido montado em base elástica com um grau de liberdade na forma adimensional, substituindo a Equação 2.24 na Equação 2.16, resultando em

$$
\begin{array}{r}
\ddot{y}+2 \zeta_{y} \dot{y}+y=\frac{U_{R}^{2}}{2 \pi^{3}\left(C_{a}+m^{*}\right)} \sqrt{1+\left(\frac{2 \pi}{U_{R}} \dot{y}\right)^{2}}\left(\frac{C_{L}^{0}}{\hat{q}_{y}} q_{y}-\frac{2 \pi}{U_{R}} C_{D} \dot{y}\right) \\
\ddot{q}_{y}+\epsilon_{y} S t U_{R}\left(q_{y}^{2}-1\right) \dot{q}_{y}+\left(S t U_{R}\right)^{2} q_{y}=A_{y} \ddot{y}
\end{array}
$$

O modelo de Facchinetti, de Langre e Biolley (2004) possui uma consideração com relação ao ângulo de ataque, onde $\beta<<1$, isto implica a linearização de $C_{V Y}$, pois se substituir a hipótese de $\beta$ muito pequeno nas Equações 2.21 e 2.22 tem-se que, $\operatorname{sen} \beta \approx \beta$ e $\cos (\beta) \approx 1$. Então utilizando dessas características do modelo, e os mesmos adimensionais vistos na Equação 2.13 tem-se que:

$$
\begin{array}{r}
\ddot{y}+2 \zeta_{y} \dot{y}+y=\frac{U_{R}^{2}}{2 \pi^{3}\left(C_{a}+m^{*}\right)}\left(\frac{C_{L}^{0}}{\hat{q}_{y}} q_{y}-\frac{2 \pi}{U_{R}} C_{D} \dot{y}\right) \\
\ddot{q}_{y}+\epsilon_{y} S t U_{R}\left(q_{y}^{2}-1\right) \dot{q}_{y}+\left(S t U_{R}\right)^{2} q_{y}=A_{y} \ddot{y}
\end{array}
$$

Foi visto na Seção 2.5 que Facchinetti, de Langre e Biolley (2004) propuseram uma única calibração dos parâmetros $A_{y}$ e $\epsilon_{y}$ para todo o fenômeno, enquanto Ogink e Metrikine (2010) propõem que sejam utilizados valores diferentes dos mesmos parâmetros tanto para o ramo upper branch quanto para o lower branch. A Tabela 2 mostra os valores utilizados dos parâmetros adotados nesta pesquisa. Como visto no trabalho de Franzini e Bunzel (2018), a transição entre os ramos upper-branch e lower-branch é definido na velocidade reduzida $U_{R}=6,5$.

Tabela 2 - Valores dos parâmetros do modelo de Ogink e Metrikine.

\begin{tabular}{c|cc}
\hline \multirow{2}{*}{ Parâmetros } & \multicolumn{2}{|c}{ Valores } \\
\cline { 2 - 3 } & Upper branch & Lower branch \\
\hline$S t$ & \multicolumn{2}{|c}{0,1932} \\
\hline$C_{L}^{0}$ & \multicolumn{2}{|c}{0,3842} \\
\hline$C_{D}$ & \multicolumn{2}{|c}{1,1856} \\
\hline$A_{y}$ & 4 & 12 \\
\hline$\varepsilon_{y}$ & 0,05 & 0,7 \\
\hline
\end{tabular}

\subsubsection{Modelo fenomenológico de Franzini e Bunzel (2018)}

O desenvolvimento do modelo fenomenológico apresentado por Franzini e Bunzel (2018) é com base na metodologia vista em Ogink e Metrikine (2010) porém com o 
incremento do grau de liberdade na direção X, que diz respeito do movimento do cilindro na direção in-line. A Figura 14 ilustra o cilindro fixo montado em base elástica que será estudado nesta Subseção.

Figura 14 - Representação do fenômeno de VIV-2GL para um cilindro rígido montado sobre apoio elástico.

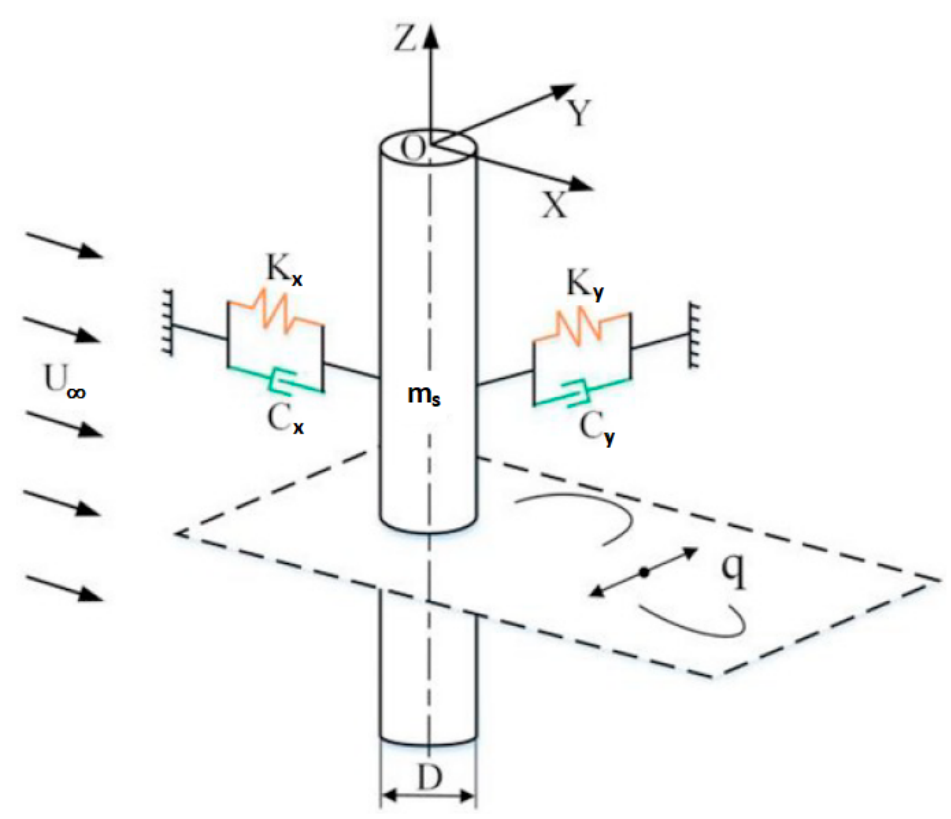

Fonte: Adaptada de Liu et al. (2020).

O modelo fenomenológico apresentado nesta Subseção utiliza duas equações de van der Pol para representar as flutuações das forças hidrodinâmicas nas duas direções. A dinâmica da esteira é representada pelas variáveis $q_{y}$ e $q_{x}$. A formulação dimensional do sistema é mostrada nas Equações 2.29, 2.30, 2.31 e 2.32.

$$
\begin{array}{r}
\left(m_{s}+m_{a}\right) \frac{d^{2} Y}{d t^{2}}+c_{y} \frac{d Y}{d t}+k_{y} Y=\frac{1}{2} \rho U_{\infty}^{2} D L C_{V Y} \\
\frac{d^{2} q_{y}}{d t^{2}}+\epsilon_{y} \omega_{s}\left(q_{y}^{2}-1\right) \frac{d q_{y}}{d t}+\omega_{s}^{2} q_{y}=\frac{A_{y}}{D} \frac{d^{2} Y}{d t^{2}} \\
\left(m_{s}+m_{a}\right) \frac{d^{2} X}{d t^{2}}+c_{x} \frac{d X}{d t}+k_{x} X=\frac{1}{2} \rho U_{\infty}^{2} D L C_{V X} \\
\frac{d^{2} q_{x}}{d t^{2}}+\epsilon_{x} \omega_{s}\left(q_{x}^{2}-1\right) \frac{d q_{x}}{d t}+\left(2 \omega_{s}\right)^{2} q_{x}=\frac{A_{x}}{D} \frac{d^{2} X}{d t^{2}}
\end{array}
$$

É importante ressaltar que, na Equação 2.32, a frequência de oscilação da força de arrasto é o dobro da frequência da emissão de vórtices $\left(2 \omega_{s}\right)$. Essa característica está relacionada com o comportamento constatado experimentalmente em cilindro fixo, como visto em Jauvtis e Williamson (2004).

O coeficiente de arrasto é representado por uma parcela flutuante acrescida de um valor médio, $\hat{C}_{D}^{0}$ e $\bar{C}_{D}^{0}$ nessa ordem, sendo os valores desses coeficientes obtidos para 
cilindro fixo. Já o coeficiente de sustentação segue a mesma metodologia vista na Equação 2.9 .

As forças hidrodinâmicas em ambas direções $F_{Y}$ e $F_{X}$ que atuam no cilindro são vistas no lado direito das Equações 2.29 e 2.31. Assim como mostra a Equação 2.12, na qual há uma superposição de efeitos na direção Y, o mesmo é feito para o caso da força na direção X conforme visto na Equação 2.33.

$$
F_{X}=F_{P O T}+F_{V X}=\frac{1}{2} \rho D L U_{\infty}^{2} C_{V X}-m_{a} \frac{d^{2} X}{d t}
$$

Na Equação 2.33 o coeficiente $C_{V X}$ representa a parcela de força na direção longitudinal que é composto pelas projeções dos coeficientes de arrasto $\left(C_{D}\right)$ e da sustentação $\left(C_{L}\right)$. A representação da decomposição de forças é mostrada na Figura 15. Com isso é possível obter as relações trigonométricas do ângulo de ataque $\beta$ nas direções in-line e cross-wise. As Equações 2.34, 2.35 e 2.37 representam as relações vistas a partir da Figura 15.

Figura 15 - Representação da decomposição das forças hidrodinâmicas em um cilindro submetido ao VIV-2GL.

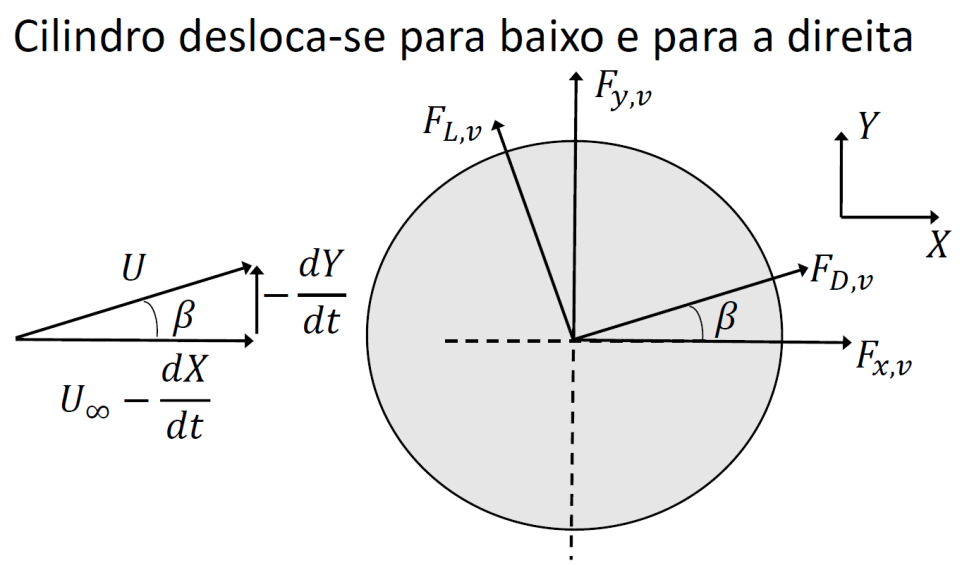

Fonte: Extraída de Franzini (2019).

Com a análise da Figura 15 é possível fazer as seguintes relações trigonométricas:

$$
\begin{array}{r}
\operatorname{sen} \beta=-\frac{\frac{d Y}{d t}}{U} \\
\cos \beta=\frac{U_{\infty}-\frac{d Y}{d t}}{U} \\
F_{V X}=F_{D} \cos \beta-F_{L} \operatorname{sen} \beta \\
U=\sqrt{\left(U_{\infty}-\frac{d X}{d t}\right)^{2}+\left(\frac{d Y}{d t}\right)^{2}}
\end{array}
$$


Analogamente ao que foi feito no modelo de VIV-1GL, são determinadas as quantidades para o modelo de VIV-2GL, conforme visto na Equação 2.38.

$$
x=\frac{X}{D} ; \quad \zeta_{x}=\frac{c_{x}}{2\left(m_{s}+m_{a}\right) \omega_{N, x}} ; \quad f^{*}=\frac{\omega_{N, x}}{\omega_{N, y}} ; \quad \omega_{N, x}=\sqrt{\frac{k_{x}}{\left(m_{s}+m_{a}\right)}}
$$

Vale ressaltar que $f^{*}$ é a relação entre as frequências naturais nas direções in-line e cross-wise.

Após apresentadas as equações das forças hidrodinâmicas atuantes no modelo VIV-2GL, é possível descrever os coeficientes de força atuante em ambas as direções in-line e cross-wise pelas seguintes equações:

$$
\begin{aligned}
C_{V X} & =\left(\frac{U}{U_{\infty}}\right)^{2}\left(C_{D V} \cos \beta-C_{L V} \operatorname{sen} \beta\right) \\
C_{V Y} & =\left(\frac{U}{U_{\infty}}\right)^{2}\left(C_{D V} \operatorname{sen} \beta+C_{L V} \cos \beta\right)
\end{aligned}
$$

O modelo matemático final adimensionalizado é feito substituindo as Equações 2.13 e 2.38 nas Equações 2.29 a 2.32. Feitas as devidas substituições chega-se ao modelo fenomenológico de Franzini e Bunzel (2018) adimensional:

$$
\begin{aligned}
& \ddot{y}+2 \zeta_{y} \dot{y}+y= \frac{1}{2 \pi^{3}} \frac{U_{R}}{\left(C_{a}+m^{*}\right)} \sqrt{1-\frac{4 \pi}{U_{R}} \dot{x}+\left(\frac{2 \pi}{U_{R}}\right)^{2}\left(\dot{x}^{2}+\dot{y}^{2}\right)} \\
& {\left[\left(U_{R}-2 \pi \dot{x}\right) \frac{C_{L}^{0}}{\hat{q_{y}}} q_{y}-2 \pi \dot{y}\left(\overline{C_{D}^{0}}+\frac{C_{D}^{0}}{\hat{q_{x}}} q_{x}\right)\right] } \\
& \ddot{q}_{y}+\epsilon_{y} S t U_{R}\left(q_{y}^{2}-1\right) \dot{q}_{y}+\left(S t U_{R}\right)^{2} q_{y}=A_{y} \ddot{y} \\
& \ddot{x}+2 \zeta_{x} f^{*} \dot{x}+\left(f^{*}\right)^{2} x= \frac{1}{2 \pi^{3}} \frac{U_{R}}{\left(C_{a}+m^{*}\right)} \sqrt{1-\frac{4 \pi}{U_{R}} \dot{x}+\left(\frac{2 \pi}{U_{R}}\right)^{2}\left(\dot{x}^{2}+\dot{y}^{2}\right)} \\
& {\left[\left(U_{R}-2 \pi \dot{x}\right)\left(\overline{C_{D}^{0}}+\frac{C_{D}^{0}}{\hat{q_{x}}} q_{x}\right)+2 \pi \dot{y}\left(\frac{C_{L}^{0}}{\hat{q_{y}}} q_{y}\right)\right] } \\
& \ddot{q_{x}}+\epsilon_{x} S t U_{R}\left(q_{x}^{2}-1\right) \dot{q}_{x}+\left(2 S t U_{R}\right)^{2} q_{x}=A_{x} \ddot{x}
\end{aligned}
$$

Assim como foi feito em Ogink e Metrikine (2010), os valores dos parâmetros $\left(\epsilon_{x}, \epsilon_{y}, A_{x}\right.$ e $\left.A_{y}\right)$ são calibrados experimentalmente para dois ramos distintos upper branch e lower branch. Como transição entre ambos ramos Franzini e Bunzel (2018) selecionaram $U_{R}=8,0$. Na Tabela 3 são observados os valores dos parâmetros utilizados pelos autores.

Uma vez detalhados os principais pontos a respeito da abordagem fenomenológica para cilindros rígidos submetidos ao VIV-1GL e VIV-2GL, faz-se necessário o levantamento da literatura sobre a excitação paramétrica, o que será abordado na Seção seguinte. 
Tabela 3 - Valores dos parâmetros do modelo de Franzini e Bunzel (2018).

\begin{tabular}{c|c|c}
\hline & Upper branch & Lower branch \\
\hline$S t$ & 0,17 & 0,17 \\
\hline$C_{L}^{0}$ & 0,3842 & 0,3842 \\
\hline$C_{D}$ & 1,1856 & 1,1856 \\
\hline$A_{x}$ & 12 & 12 \\
\hline$\varepsilon_{x}$ & 0,6 & 0,7 \\
\hline$A_{y}$ & 2 & 12 \\
\hline$\varepsilon_{y}$ & $0,0045 e^{0,228 m^{*}}$ & 0,7 \\
\hline
\end{tabular}

\subsection{Excitação paramétrica}

O problema de excitação paramétrica é um problema clássico em dinâmica de estruturas. Esse problema ocorre quando pelo menos um dos parâmetros da equação de movimento depende explicitamente do tempo. Nesta pesquisa, o termo dependente do tempo é a rigidez, que varia harmonicamente com o tempo.

O estudo de excitação paramétrica supostamente iniciou-se com Faraday. Foi observado por ele que o comportamento das ondas de superfície em um recipiente cilíndrico cheio de fluido, sob ação de excitações verticais oscilava com dobro da frequência de excitação.

A equação de movimento de sistemas excitados parametricamente é formada por equações que possuem coeficientes variando no tempo, como é visto na equação de Hill mostrada na Equação 2.45.

$$
\frac{d^{2} u}{d t^{2}}+u(t) p(t)=0
$$

onde p é uma função periódica

Um caso caso particular da equação de Hill é a equação de Mathieu, que ocorre quando a função $p(t)$ varia harmonicamente com o tempo, conforme visto na Equação 2.46 .

$$
\ddot{u}+(\delta+2 \epsilon \cos (2 t)) u=0
$$

Para descrever as regiões de estabilidade e instabilidade (hachuradas) ou regiões de instabilidade paramétrica, utiliza-se o diagrama de Strutt, visto na Figura 16. Em Nayfeh e Mook (1979) e Meirovitch (2003) é possível ver deduções das curvas de transição do diagrama. Comumente utilizam métodos matemáticos, como método das múltiplas escalas 
para determinar quais são as regiões de instabilidade no diagrama de Strutt. A Teoria de Floquet mostra algumas conclusões a respeito da Equação 2.46. Dentre elas, verifica-se que, para determinados pares $(\delta, \epsilon)$, a solução cresce de forma ilimitada ao longo do tempo, porém para outros, a solução é limitada.

Figura 16 - Diagrama de Strutt.

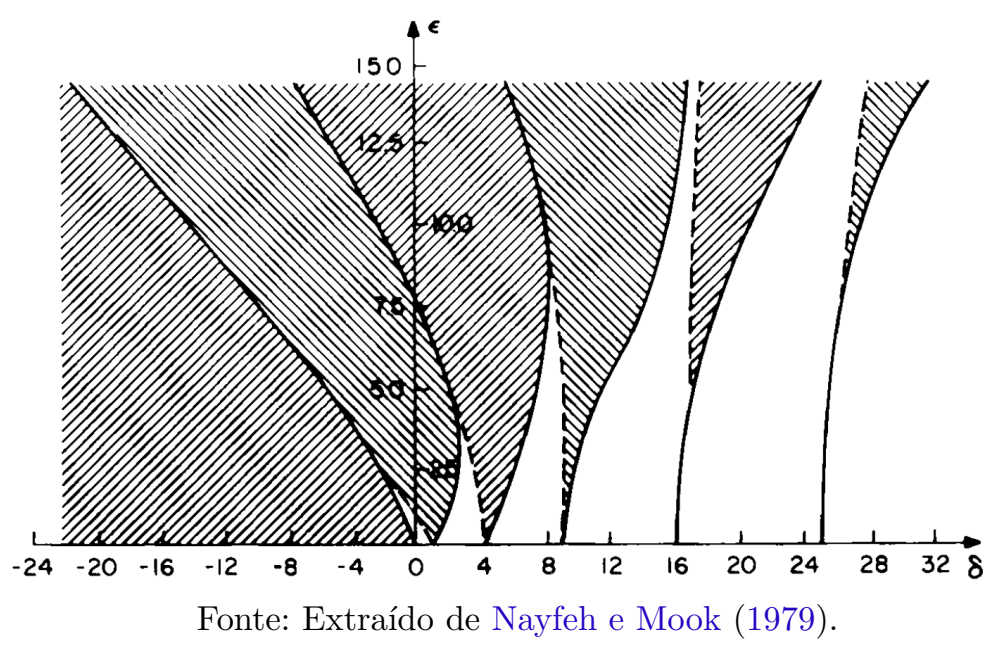

O fenômeno da ressonância clássica, ocorre em oscilações forçadas quando a frequência da força externa atuante se aproxima da frequência natural do sistema. De acordo com Butikov (1999) a ressonância paramétrica possui causas e características diferentes da ressonância clássica, especialmente quando a frequência de excitação paramétrica é igual ao dobro da frequência natural do sistema, ou seja, condição de instabilidade paramétrica principal.

O estudo de estabilidade envolvendo excitação paramétrica tem grande importância no estudo envolvendo a dinâmica de risers, pois se trata de estruturas esbeltas, presas a uma das extremidades e submetida a movimentos de topo proveniente de ondas de superfície. Isso faz com que surja uma força de tração variável no tempo que altera a rigidez geométrica do riser ao longo do tempo.

O problema envolvendo excitação paramétrica foi estudado por diversos pesquisadores no âmbito da engenharia oceânica. Dentre muitos trabalhos, devem-se ressaltar Patel e Park (1991) no qual os autores estudaram o comportamento dinâmico dos tendões de uma TLP submetidos a excitação paramétrica, para grandes valores dos parâmetros $(\delta, \epsilon)$ da equação de Mathieu. Thampi e Niedzwecki (1992) estudaram as respostas não lineares dos risers sob os efeitos da excitação paramétrica utilizando modelos estocásticos. Chatjigeorgiou e Mavrakos (2002) analisaram a resposta dinâmica na direção transversal de estruturas longas e esbeltas sujeitas a excitação paramétrica no topo da estrutura. Maiores detalhes sobre a excitação paramétrica em risers podem ser encontrados nos artigos Simos e Pesce (1997), Franzini e Mazzilli (2016), Mazzilli, Rizza e Dias (2016) e 
Vernizzi, Franzini e Lenci (2019)

\subsection{Problema combinado de excitação paramétrica e VIV}

O estudo combinado dos efeitos de excitação paramétrica e VIV possui grande importância na engenharia offshore, principalmente na dinâmica de risers. Por causa da esbeltez dessa estrutura, já que a rigidez geométrica que está relacionada à força normal é predominante sobre a rigidez devida à flexão. Além disso os fenômenos são danosos a estrutura, por terem relações com à fadiga estrutural.

Um dos primeiros trabalhos a abordar os efeitos combinados foi Silveira et al. (2007), que utilizaram um modelo numérico para simular a dinâmica de risers utilizando MEF acoplado ao modelo fenomenológico do tipo van der Pol, sendo imposta uma tração no topo do cabo para cada instante de tempo do modelo. Os autores perceberam claramente uma modulação na amplitude de resposta e na frequência devido a variação da tração.

Em Franzini et al. (2015), foram apresentados os resultados experimentais para um cilindro flexível semi-submerso sujeito ao VIV. Nesse trabalho, foi imposto um movimento de topo que varia harmonicamente com amplitude de movimento constante $A_{t} / L_{0}=1 \%$, sendo $L_{0}$ o comprimento indeformado do cilindro. Os autores estudaram três casos específicos da frequência de movimento $\left(f_{t}\right)$, sendo eles $f_{t}: f_{N, 1}=1: 1, f_{t}: f_{N, 1}=1: 2$ e $f_{t}: f_{N, 1}=1: 3$ onde $f_{N, 1}$ corresponde a frequência natural do primeiro modo de vibrar. Os autores concluíram que a resposta do modelo sujeito ao efeito concomitante de VIV e excitação paramétrica é diferente daquela em que o cilindro flexível é submetido somente ao VIV.

Segundo o estudo numérico de Yuan, Xue e Tang (2018), os autores sugeriram um método de decomposição de força como um modelo alternativo para prever a resposta do VIV na direção cross-wise em um cilindro flexível submetido a uma variação temporal da força de tração no topo. Além disso, os autores enfatizaram que a resposta estrutural sob efeitos combinados de VIV e excitação paramétrica foi aumentado (quando comparada ao caso sem excitação paramétrica) quando ocorre a instabilidade paramétrica principal $\left(f_{t}: f_{N, 1}=2: 1\right)$. Os autores ainda compararam o modelo proposto com resultados experimentais de Franzini et al. (2016) e verificaram que o modelo foi capaz de recuperar alguns aspectos qualitativos e até mesmo quantitativos da resposta do cilindro.

Franzini et al. (2018) apresentam investigações experimentais a respeito da excitação paramétrica em um cilindro flexível totalmente submerso, concomitante com os efeitos de VIV. Nesse trabalho, devido à concomitância entre os fenômenos, a resposta do cilindro possui modulações significativas em amplitude, o que não foi observado para condição na qual o cilindro excitado somente pelo VIV. Dentre outras conclusões, os autores observaram que para o caso específico em que o movimento imposto foi $f_{t}: f_{N, 1}=2: 1$, ou seja, a frequência de excitação paramétrica era duas vezes a frequência natural do primeiro modo, 
essa instabilidade paramétrica acarretou em um aumento considerável nas amplitudes de oscilação do cilindro.

O trabalho de Ma et al. (2020) fez uma investigação experimental sobre o comportamento de um cilindro flexível horizontal sujeito à variação temporal da tração concomitante ao fenômeno de VIV. Os autores estudaram a resposta do cilindro sob o efeito combinado para diversas variações de amplitudes e frequências de excitação. Um resultado interessante obtido pelos autores mostrou que para valores da relação de frequência $f_{t} / f_{N, 1}$ diferentes de $f_{t} / f_{N, 1}=2,0$, foi possível notar através da análise espectral o surgimento da "frequência soma" e da "frequência diferença". Os autores também observaram que para esse caso específico de excitação paramétrica, também houve um aumento nas amplitudes de oscilação do cilindro.

Deve-se ressaltar a importância dos efeitos combinados de VIV e excitação paramétrica, pois esses efeitos agindo concomitantemente podem levar o sistema a maiores respostas dinâmicas do que se analisados separadamente.

\subsection{Lacunas de conhecimento na literatura}

Como mostrado na revisão bibliográfica desse trabalho, deve-se ressaltar a importância dos efeitos combinados de VIV e excitação paramétrica, pois esses efeitos agindo concomitantemente podem levar o sistema a maiores respostas dinâmicas do que se analisados separadamente. Os trabalhos encontrados na literatura que dizem respeito sobre esse problema são resultados experimentais envolvendo cilindros flexíveis.

Após feita a presente revisão bibliográfica notou-se que existem algumas lacunas de conhecimento, conforme apresentado a seguir:

- O estudo numérico de excitação paramétrica combinado ao fenômeno de VIV em cilindro rígido montado em base elástica, utilizando modelo fenomenológico;

- Resultados experimentais envolvendo cilindro rígido montado em base elástica, com excitação paramétrica e submetido ao VIV, correlacionando com os dados experimentais de cilindro flexível.

- Cálculo numérico da força hidrodinâmica no cilindro, sujeito ao efeito de VIV concomitante com excitação paramétrica.

Esta dissertação tem importância para as aplicações tecnológicas que lidam com os efeitos concomitantes dos fenômenos de VIV e excitação paramétrica em um cilindro rígido montado em base elástica, auxiliando nas primeiras etapas de análise com o objetivo de entender o comportamento de modelos de maior hierarquia. 


\section{Modelagem matemática e metodologia de simulação}

Este capítulo apresenta os modelos matemáticos e a respectiva metodologia de simulação focando no problema de cilindro rígido com um grau de liberdade e com dois graus de liberdade (VIV-1GL e VIV-2GL, respectivamente). Montados sobre apoio elástico e submetidos à concomitância entre fenômenos de VIV e excitação paramétrica como ilustrado na Figura 17.

O cilindro possui massa $m_{s}$, diâmetro $\mathrm{D}$, imerso em fluido com massa especifica $\rho$ e com escoamento uniforme de velocidade $U_{\infty}$ e com comprimento L. Para esse modelo, o sistema foi descrito com a variação de rigidez ao longo do tempo $k(t)=\bar{k}+\Delta k \cos (\Omega t)$, sendo que $\Delta k$ significa a amplitude de excitação, $\Omega$ a frequência de variação da rigidez e livre para oscilar em ambas as direções in-line e cross-wise.

Figura 17 - Representação do modelo em estudo. A aceleração da gravidade é perpendicular ao plano xy.

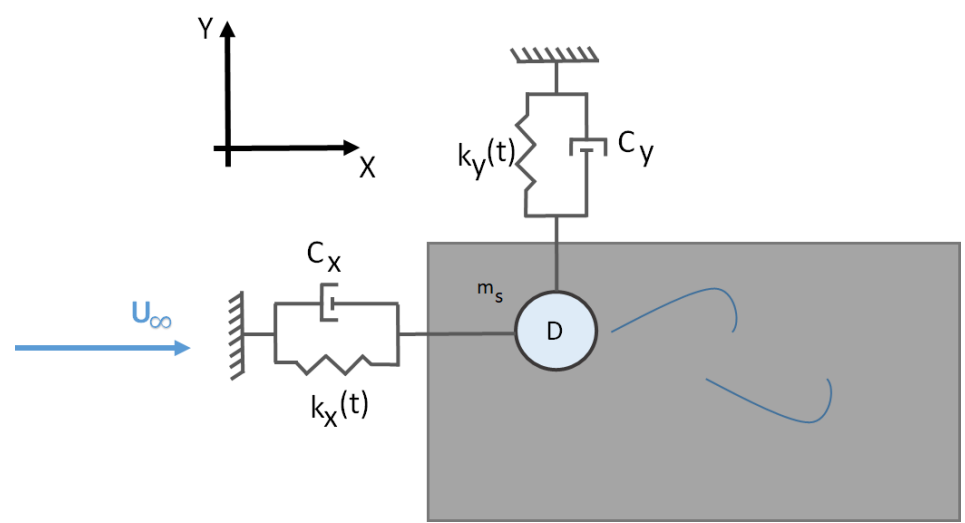

Fonte: O autor

Os modelos utilizados nesta pesquisa são com base no oscilador do tipo van der Pol, onde os carregamentos para as condições VIV-1GL e VIV-2GL, são propostos por Ogink e Metrikine (2010) e Franzini e Bunzel (2018) respectivamente. 


\subsection{Equações governantes para o problema VIV-1GL concomitante com a excitação paramétrica}

Nesta Subseção, são apresentadas as equação que governam o movimento do cilindro rígido montado sobre base elástica para o problema VIV-1GL.

As equações dimensionais do sistema são dadas por:

$$
\begin{array}{r}
\left(m_{s}+m_{a}\right) \frac{d^{2} Y}{d t^{2}}+c_{y} \frac{d Y}{d t}+\left(\overline{k_{y}}+\Delta k_{y} \cos \left(\Omega_{y} t\right)\right) Y=\frac{1}{2} \rho U_{\infty}^{2} D L C_{V Y} \\
\frac{d^{2} q_{y}}{d t^{2}}+\varepsilon_{y} \omega_{s}\left(q_{y}^{2}-1\right) \frac{d q_{y}}{d t}+\omega_{s}^{2} q_{y}=\frac{A_{y}}{D} \frac{d^{2} Y}{d t^{2}}
\end{array}
$$

A componente de força hidrodinâmica na direção cross-wise $C_{V Y}$ é decomposta em dois termos, uma parte devido ao coeficiente de sustentação $\left(C_{L}\right)$ e outra parte devido o coeficiente de arrasto $\left(C_{D, V}\right)$ conforme visto na Equação 2.19.

Com intuito de ter uma maior generalidade das análises, as Equações 3.1 e 3.2 são reescritas na forma adimensional. Pode-se notar que os parâmetros que governam a excitação paramétrica são $\delta$ e $n_{y}$, correspondente à amplitude e frequência de excitação, respectivamente. Lembrando que alguns dos adimensionais já foram apresentados no Capítulo 2. Para tanto, considere as seguintes quantidades:

$$
\begin{gathered}
y=\frac{Y}{D} ; \quad m^{*}=\frac{m_{s}}{m_{d}} ; \quad U_{R}=\frac{2 \pi U_{\infty}}{\omega_{N_{y}} D} ; \\
\zeta_{y}=\frac{c_{y}}{2\left(m_{s}+m_{a}\right) \omega_{N_{y}}} ; \quad S t=\frac{f_{s} D}{U_{\infty}} ; \quad C_{a}=\frac{m_{a}}{m_{d}} \\
\delta_{y}=\frac{\Delta k_{y}}{k_{y}} ; \quad n_{y}=\frac{\Omega_{y}}{\omega_{N_{y}}} ;
\end{gathered}
$$

onde $\omega_{N_{y}}=\sqrt{\frac{\overline{k_{y}}}{\left(m_{s}+m_{a}\right)}}$ é a frequência natural do cilindro. Introduzindo as quantidades propostas na Equação 3.4 nas Equações 3.1 e 3.2 e adotando $(\dot{)})=\frac{d}{d \tau}$ ( ) como sendo a derivada com relação ao tempo adimensional $\tau$, são obtidas as seguintes equações adimensionais:

$$
\begin{gathered}
\ddot{y}+2 \zeta_{y} \dot{y}+\left(1+\delta_{y} \cos \left(n_{y} \tau\right)\right) y=\frac{U_{R}^{2}}{2 \pi^{3}\left(m^{*}+C_{a}\right)} \sqrt{1+\left(\frac{2 \pi}{U_{R}} \dot{y}\right)^{2}}\left(\frac{C_{L}^{0}}{\hat{q}_{y}} q_{y}-\frac{2 \pi}{U_{R}} C_{D, V} \dot{y}\right) \\
\ddot{q}_{y}+\epsilon_{y} S t U_{R}\left(q_{y}^{2}-1\right) \dot{q}_{y}+\left(S t U_{R}\right)^{2} q_{y}=A_{y} \ddot{y}
\end{gathered}
$$


Apresentadas as equações de movimento adimensionais, é dada ênfase às forças hidrodinâmicas atuando em um cilindro sujeito ao VIV. De acordo com o modelo de Ogink e Metrikine (2010) a força hidrodinâmica $F_{V Y}$ pode ser calculada separando a parcela devido ao escoamento potencial e outra devido a emissão de vórtices. O esquema mostrado na Figura 13, ilustra a decomposição de forças utilizando relações trigonométricas, então as seguintes Equações 3.6 e 3.7 são obtidas:

$$
\begin{aligned}
& F_{v, x}=F_{D, v} \cos (\beta)-F_{L, v} \operatorname{sen}(\beta) \\
& F_{v, y}=F_{D, v} \operatorname{sen}(\beta)+F_{L, v} \cos (\beta)
\end{aligned}
$$

É importante ressaltar que a força transversal atuando no cilindro devida à emissão de vórtices aparece no lado direito da Equação 3.1, com $C_{V Y}$ sendo o coeficiente correspondente. Deve-se lembrar que a outra parte da força transversal total está associada a massa adicional, que já foi incluída no lado esquerdo da mesma equação. Consequentemente, os coeficientes de força nas direções transversal e longitudinal totais são vistos nas Equações 3.8 e 3.9, como deduzido em Ueno e Franzini (2019).

$$
\begin{gathered}
C_{y}=C_{V Y}-\frac{2 C_{a} \pi^{3}}{U_{R}^{2}} \ddot{y} \\
C_{x}=\sqrt{1+\left(\frac{2 \pi}{U_{R}} \dot{y}\right)^{2}}\left(\frac{2 \pi}{U_{R}} C_{L, v} \dot{y}+C_{D, v}\right)
\end{gathered}
$$

Depois de tomar as equações de movimento adimensionais e as equações de forças hidrodinâmicas para o sistema de VIV-1GL, o texto segue para a apresentação do modelo VIV-2GL.

\subsection{Equações governantes para o modelo VIV-2GL concomitante com a excitação paramétrica}

Analogamente ao que foi apresentado na Subseção anterior para o problema VIV$1 \mathrm{GL}$, aqui serão apresentadas as equações de movimento para o modelo na condição VIV2GL. O carregamento hidrodinâmico para essa condição considera o modelo apresentado por Franzini e Bunzel (2018) na Seção 2.5.2 deste trabalho.

O modelo matemático para a condição VIV-2GL e excitação paramétrica é representado na forma dimensional pelas Equações 3.10, 3.11, 3.12 e 3.13. 


$$
\begin{array}{r}
\left(m_{s}+m_{a}\right) \frac{d^{2} Y}{d t^{2}}+c_{y} \frac{d Y}{d t}+\left(\overline{k_{y}}+\Delta k_{y} \cos \left(\Omega_{y} t+\theta\right)\right) Y=\frac{1}{2} \rho U_{\infty}^{2} D L C_{V Y} \\
\frac{d^{2} q_{y}}{d t^{2}}+\epsilon_{y} \omega_{s}\left(q_{y}^{2}-1\right) \frac{d q_{y}}{d t}+\omega_{s}^{2} q_{y}=\frac{A_{y}}{D} \frac{d^{2} Y}{d t^{2}} \\
\left(m_{s}+m_{a}\right) \frac{d^{2} X}{d t^{2}}+c_{x} \frac{d X}{d t}+\left(\overline{k_{x}}+\Delta k_{x} \cos \left(\Omega_{x} t\right)\right) X=\frac{1}{2} \rho U_{\infty}^{2} D L C_{V X} \\
\frac{d^{2} q_{x}}{d t^{2}}+\epsilon_{x} \omega_{s}\left(q_{x}^{2}-1\right) \frac{d q_{x}}{d t}+\left(2 \omega_{s}\right)^{2} q_{x}=\frac{A_{x}}{D} \frac{d^{2} X}{d t^{2}}
\end{array}
$$

Mais uma vez, tendo em vista o uso dos adimensionais já apontadas nas Equações 3.4 e 2.38 faz-se o acréscimo das seguintes quantidades:

$$
x=\frac{X}{D} ; \quad \zeta_{x}=\frac{c_{x}}{2\left(m_{s}+m_{a}\right) \omega_{N, x}} ; \quad \delta_{x}=\frac{\Delta k_{x}}{\overline{k_{x}}} ; \quad n_{x}=\frac{\Omega_{x}}{\omega_{N, x}} ; \quad f^{*}=\frac{\omega_{N, x}}{\omega_{N, y}}
$$

sendo $\omega_{N, x}$ a frequência natural do cilindro na direção in-line, determinada por $\omega_{N, x}=\sqrt{\frac{k_{x}}{\left(m_{s}+m_{a}\right)}}$;

Substituindo as quantidades definidas nas Equações 3.4 e 3.14 nas Equações 3.10 a 3.13, o modelo matemático para a condição VIV-2GL é escrito em sua forma adimensional como:

$$
\begin{array}{r}
\ddot{y}+2 \zeta_{y} \dot{y}+\left[1+\delta_{y} \cos \left(n_{y} \tau\right)\right] y=\frac{1}{2 \pi^{3}} \frac{U_{R}}{\left(C_{a}+m^{*}\right)} C_{V Y} \\
\ddot{q}_{y}+\epsilon_{y} S t U_{R}\left(q_{y}^{2}-1\right) \dot{q}_{y}+\left(S t U_{R}\right)^{2} q_{y}=A_{y} \ddot{y} \\
\ddot{x}+2 \zeta_{x} f^{*} \dot{x}+\left(f^{*}\right)^{2}\left[1+\delta_{x} \cos \left(n_{x} \tau\right)\right] x=\frac{1}{2 \pi^{3}} \frac{U_{R}}{\left(C_{a}+m^{*}\right)} C_{V X} \\
\ddot{q}_{x}+\epsilon_{x} S t U_{R}\left(q_{x}^{2}-1\right) \dot{q}_{x}+\left(2 S t U_{R}\right)^{2} q_{x}=A_{x} \ddot{x}
\end{array}
$$

Seguindo a mesma metodologia apresentada para a condição VIV-1GL, as forças de sustentação $F_{L, v}$ e de arrasto $F_{D, v}$ que estão relacionadas com a emissão de vórtices são decompostas nas direções cross-wise e in-line, conforme visto nas Equações 3.6 e 3.7. Através das forças hidrodinâmicas, é possível obter os coeficientes de forças nas duas direções $C_{V Y}$ e $C_{V X}$, como:

$$
\begin{aligned}
& C_{V Y}=\sqrt{1-\frac{4 \pi}{U_{R}} \dot{x}+\left(\frac{2 \pi}{U_{R}}\right)^{2}\left(\dot{x}^{2}+\dot{y}^{2}\right)}\left[C_{L}\left(1-\frac{2 \pi f^{*} \dot{x}}{U_{R}}\right)-C_{D} \frac{2 \pi}{U_{R}} \dot{y}\right] \\
& C_{V X}=\sqrt{1-\frac{4 \pi}{U_{R}} \dot{x}+\left(\frac{2 \pi}{U_{R}}\right)^{2}\left(\dot{x}^{2}+\dot{y}^{2}\right)}\left[C_{L} \frac{2 \pi}{U_{R}} \dot{y}+C_{D}\left(1-\frac{2 \pi f^{*} \dot{x}}{U_{R}}\right)\right]
\end{aligned}
$$


Expandindo-se, o que foi visto em Ueno e Franzini (2019) e apresentado na Subseção 3.1, agora para a condição VIV-2GL. São obtidos os coeficientes de força totais nas direções cross-wise e in-line $\left(C_{y}\right.$ e $\left.C_{x}\right)$, conforme as Equações 3.21 e 3.22.

$$
\begin{aligned}
& C_{y}=C_{V Y}-\frac{2 C_{a} \pi^{3}}{U_{R}^{2}} \ddot{y} \\
& C_{x}=C_{V X}-\frac{2 C_{a} \pi^{3}}{U_{R}^{2}} \ddot{x}
\end{aligned}
$$

Na Seção seguinte, será mostrada a metodologia de análise desenvolvida para obter a resolução numérica dos modelos apresentados.

\subsection{Metodologia}

Nesta Seção, é apresentada a metodologia empregada para análise numérica, dos efeitos da excitação paramétrica combinada com fenômeno de VIV em cilindros rígidos montados em base elástica nas condições VIV-1GL e VIV-2GL.

As Equações de movimento 3.4 e 3.5 (referentes a condição VIV-1GL) e as Equações 3.15 a 3.18 (referentes a condição VIV-2GL) foram integradas numericamente utilizando o método Runge-Kutta de quarta ordem, que está implementado no programa Matlab ${ }^{\circledR}$ por meio da função ode45. O tempo máximo de simulação é $\tau_{m a ́ x}=800$. As condições inciais são $y(0)=0, \dot{y}(0)=0, q_{y}(0)=0,1, \dot{q}_{y}(0)=0$ para condição VIV-1GL; e $q_{y}(0)=0,01$, $\dot{q}_{y}(0)=0$ na condição VIV-2GL.

O sistema proposto nessa pesquisa foi estudado somente para o intervalo de velocidade reduzida $2,0 \leq U_{R} \leq 14,0$, sendo discretizado com incrementos de velocidade reduzida de $\Delta U_{R}=0,1$. Os valores das amplitudes características do cilindro nas direção cross-wise e in-line $\left(\hat{A_{y}}\right.$ e $\hat{A_{x}}$ ) calculados são obtidos através das Equações 3.23 e 3.24, onde $\sigma_{y}$ e $\sigma_{x}$ correspondem ao desvio padrão da série temporal, em regime estacionário definido pelo intervalo de tempo $\tau>\tau_{\text {máx }} / 2$.

$$
\begin{aligned}
& \hat{A}_{y}=\sqrt{2} \sigma_{y} \\
& \hat{A}_{x}=\sqrt{2} \sigma_{x}
\end{aligned}
$$

Os parâmetros de calibração do modelo fenomenológico são os mesmos apresentados em Ogink e Metrikine (2010) e Franzini e Bunzel (2018), conforme visto nas Seções 2.5.1 e 2.5.2. Com relação aos parâmetros estruturais de massa reduzida $\left(m^{*}\right)$ e taxa de amortecimento $(\zeta)$, foram adotados para condição VIV-1GL, $m^{*}=2,6$ e $\zeta_{y}=0,0008$ 
conforme descrito no experimento de Franzini et al. (2012)2 . Já para a condição VIV-2GL, o parâmetro de massa reduzida foi mantido, enquanto $\zeta_{y}=\zeta_{x}=0,0007$ e $f^{*}=1,0$. Tendo em vista que no problema do riser há uma variação temporal da rigidez, devido a rigidez geométrica sofrer alterações em consequência da variação temporal da força normal. Assume-se nesta pesquisa que $\Delta k_{x}=\Delta k_{y}, \overline{k_{x}}=\overline{k_{y}}$ e $\theta=0^{\circ}$.

Outra condição presente nas curvas de amplitude característica é o resultado dos modelos fenomenológicos de Ogink e Metrikine (2010) e Franzini e Bunzel (2018) submetidos somente ao fenômeno de VIV, indicando o sistema sem excitação paramétrica $\left(\right.$ delt $_{x}=$ delta $\left._{y}=0\right)$, denominado de "VIV Puro". Cabe aqui ressaltar que foi definido como ponto de partida dos resultados a amplitude de excitação paramétrica como $\delta_{y}=\delta_{x}=0,10$. O trabalho também faz um estudo de sensibilidade da resposta do sistema hidro-elástico com relação a variação dos parâmetros $(n$ e $\delta$ ) que definem a excitação paramétrica. Além disso, são feitas análises da influência da variação de $\delta$ nos coeficientes de força $\left(C_{y}\right.$ e $\left.C_{x}\right)$ nas direções cross-wise e in-line.

Neste trabalho, para algumas análises foi utilizada a transformada de HilbertHuang (HHT), que pode ser encontrada em Huang et al. (1998). Essa técnica é aplicada ao fenômeno de VIV, tendo em vista algumas características como saltos de resposta, comportamento não estacionário e não linear, além da modulação de frequência. A HHT foi utilizada nos seguintes artigos no contexto de VIV Pesce, Fujarra e Kubota (2006), Gonçalves et al. (2012), e Franzini et al. (2015).

Por último foi feita uma análise assintótica das equações de movimento para a condição VIV-1GL utilizando o método das múltiplas escalas. Maiores detalhes sobre o método podem ser encontrados em Nayfeh e Mook (1979).

$\overline{2}$ Lembrando que o experimento não leva em consideração os efeitos de excitação paramétrica. 


\section{Resultados e discussões}

Neste Capítulo são mostrados os principais resultados obtidos com as simulações numéricas realizadas. Foram analisadas as curvas de amplitude característica do cilindro em função da velocidade reduzida, variando a frequência adimensional de excitação paramétrica $n_{x}=n_{y}$ e a amplitude de excitação $\delta_{y}=\delta_{x}$, para os casos VIV-1GL e VIV-2GL. Ambos os resultados são comparados com os dados experimentais e resultados numéricos de cilindros flexíveis e submetidos a variação da força de tração no topo. Essa comparação foi feita de maneira a discutir quais os aspectos qualitativos e até quantitativos foram recuperados com os modelos de baixa hierarquia (menor número de graus de liberdade).

É importante enfatizar que nos experimentos apresentados em Franzini et al. (2018), o deslocamento estático no topo (responsável pela tração média no topo $\bar{N}$ ) foi $\Delta L=50 \mathrm{~mm}$ e a amplitude de excitação paramétrica $\left(A_{t}\right)$ é $1 \%$ do comprimento não deformado do cilindro $\left(L_{0}=2552 \mathrm{~mm}\right)$, isso dá um indicativo da variação de tração no cilindro flexível de $\Delta N / \bar{N}=A_{t} / \Delta L=25,52 / 50,00 \approx 50 \%$ na variação de rigidez. Portanto, a excitação paramétrica adimensional obtida nos experimentos é 5 vezes maior que o valor aqui investigado $\left(\delta_{y}=0,10\right)$. Note que no trabalho de Yuan, Xue e Tang (2018) os autores utilizaram $\Delta N / \bar{N}=10 \%$. A Seção 4.1 apresenta os resultados numéricos para a condição VIV-1GL. Por sua vez, a Seção 4.2 traz os resultados numéricos considerando a condição VIV-2GL.

\subsection{Resultados numéricos VIV-1GL}

Nesta Seção são analisadas as curvas de amplitude característica do cilindro em função da velocidade reduzida, variando a frequência adimensional de excitação paramétrica $n_{y}$ e a amplitude $\delta_{y}$. De maneira adicional, são mostradas as séries temporais de deslocamento do cilindro. Após essa análise, faz-se a aplicação da Transformada de Hilbert-Huang, o que traz informações complementares à análise baseada na Transformada de Fourier.

\subsubsection{Resultados considerando $n_{y}=1$}

A Figura 18 mostra a curva de amplitude característica de oscilação do cilindro como função da velocidade reduzida para o caso $n_{y}=1$, variando a amplitude de excitação paramétrica $\delta_{y}$ de 0,02 a 0,12 . Note que a excitação paramétrica não levou à nenhuma mudança na curva de amplitude característica para toda faixa de velocidades reduzidas características do lock-in. 
Figura 18 - Curvas de respostas da amplitude característica

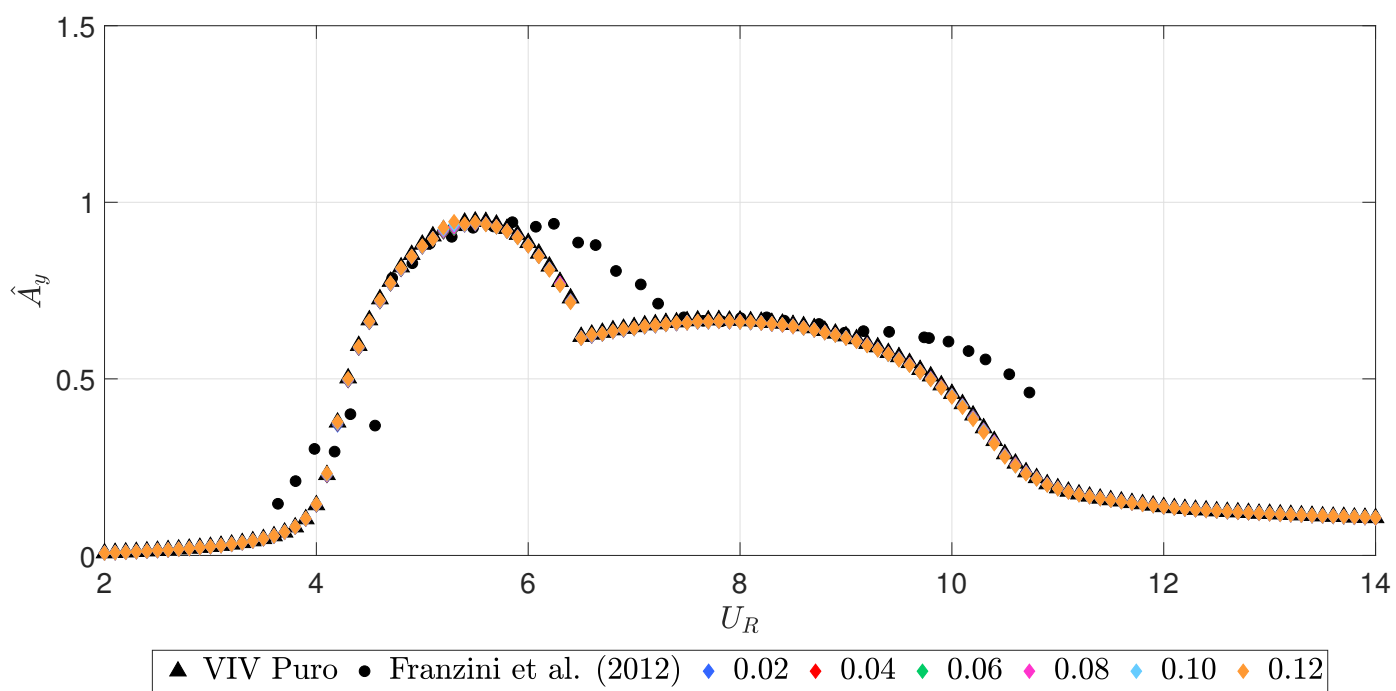

Fonte: O autor.

A Figura 19 apresenta os envelopes de amplitude característica de oscilação na direção cross-wise para $U_{R}=4,99$ do experimento de cilindro flexível apresentado em Franzini et al. $(2015)^{3}$. Como mostra a Figura 19a para condição "VIV Puro", a máxima amplitude de resposta é $\max \left\{\hat{A}_{y}\right\} \approx 0,80$ e ocorre próximo ao meio do vão. Já na Figura 19b sob o efeito combinado de VIV e excitação paramétrica, revela que a máxima amplitude de oscilação $\max \left\{\hat{A}_{y}\right\} \approx 1,00$, o que indica uma amplificação da resposta devido a excitação paramétrica.

Figura 19 - Envelopes de amplitude característica de oscilação. $U_{R}=4,99$

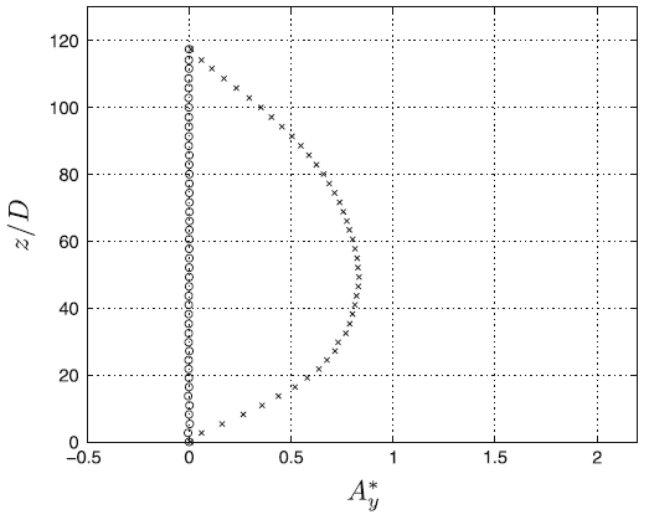

(a) Sistema sem Excitação Paramétrica.

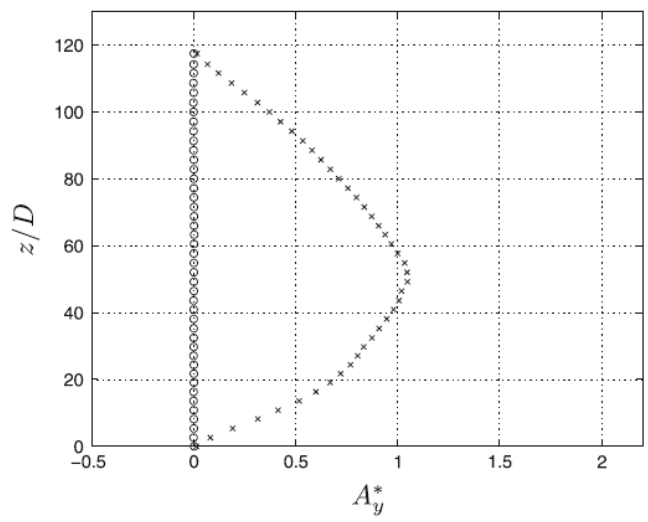

(b) Sistema com Excitação Paramétrica.

Fonte: Extraído de Franzini et al. (2015)

3 Experimento conduzido com cilindro semi-imerso. 
Comparando os resultados obtidos do modelo VIV-1GL para o caso de excitação paramétrica $\left(n_{y}=1\right)$ com os resultados advindos dos experimentos de cilindro flexível para velocidades reduzidas $U_{R}<6,5$, percebe-se que o modelo fenomenológico não foi capaz de recuperar o ganho de amplitude $\hat{A}_{y}$ visto no cilindro flexível. Isso pode ser um indício da dominância do fenômeno de VIV sob a excitação paramétrica.

Em sequência, analisam-se as séries temporais $y(\tau)$ de deslocamento do cilindro e os correspondentes espectros de amplitude $\hat{A}(\hat{f})$ para as condições com e sem excitação paramétrica. Essa última condição, aqui denominada de "VIV Puro"corresponde a $\delta_{y}=0$. Além disso, a amplitude característica de oscilação na condição "VIV Puro"será representada por linhas tracejadas em vermelho nas séries temporais para condição combinada de VIV com excitação paramétrica.

As Figuras 20a e 20b, apresentam as séries temporais de deslocamento $y(\tau)$ para a condição "VIV PURO"das seguintes velocidades reduzidas $U_{R}=5,5$ e $U_{R}=5,8$, respectivamente. Percebe-se que as respostas do cilindro são livres de modulação em amplitude, sendo os espectros de amplitude caracterizados por uma frequência adimensional de $\hat{f}=1,0$ com banda estreita.

Figura 20 - Series temporais de resposta do cilindro para o caso sem excitação paramétrica
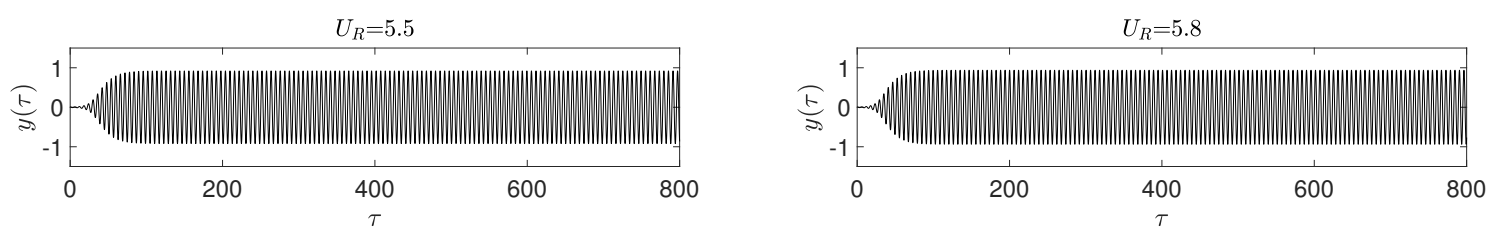

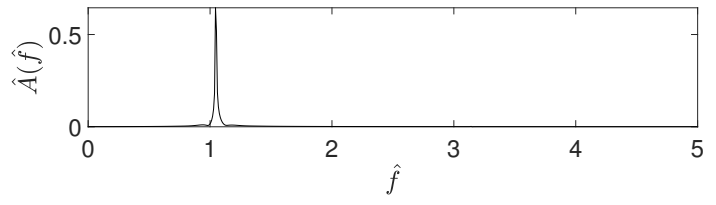

(a) $U_{R}=5,5$.

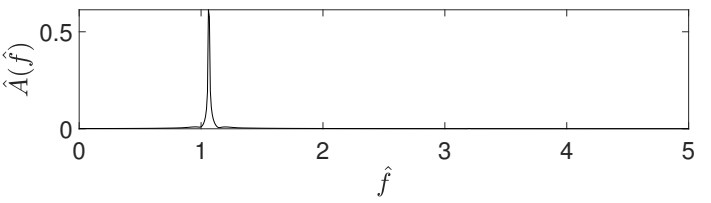

(b) $U_{R}=5,8$.

Fonte: O autor.

A Figura 21 ilustra as séries temporais $y(\tau)$ obtidas para o caso de excitação paramétrica $n_{y}=1$ e $\delta_{y}=0,10$. A análise dessas séries temporais revela que, ao contrário do que foi visto no caso "VIV Puro"(ver Figura 20), o efeito concomitante de VIV e excitação paramétrica alterou a resposta do cilindro. As duas séries temporais possuem modulações em amplitude visualmente muito pequenas e possuem amplificação da resposta que é muito inferior àquela do cilindro flexível. Aqui, as séries temporais do VIV-1GL mostram que amplitude característica é aumentada de $\hat{A}_{y}=0,92$, na condição "VIV Puro", para $\hat{A}_{y}=0,98$ em $U_{R}=5,5$ e $\hat{A}_{y}=0,90$ "VIV Puro"para $\hat{A}_{y}=0,96$ em $U_{R}=5,8$. Em relação aos espectros de amplitude $\hat{A}(\hat{f})$, nota-se que ambos possuem banda estreita com energia concentrada em $\hat{f}=1,0$. 
Figura 21 - Series temporais de resposta do cilindro para o caso $\delta_{y}=0,10$ e $n_{y}=1$
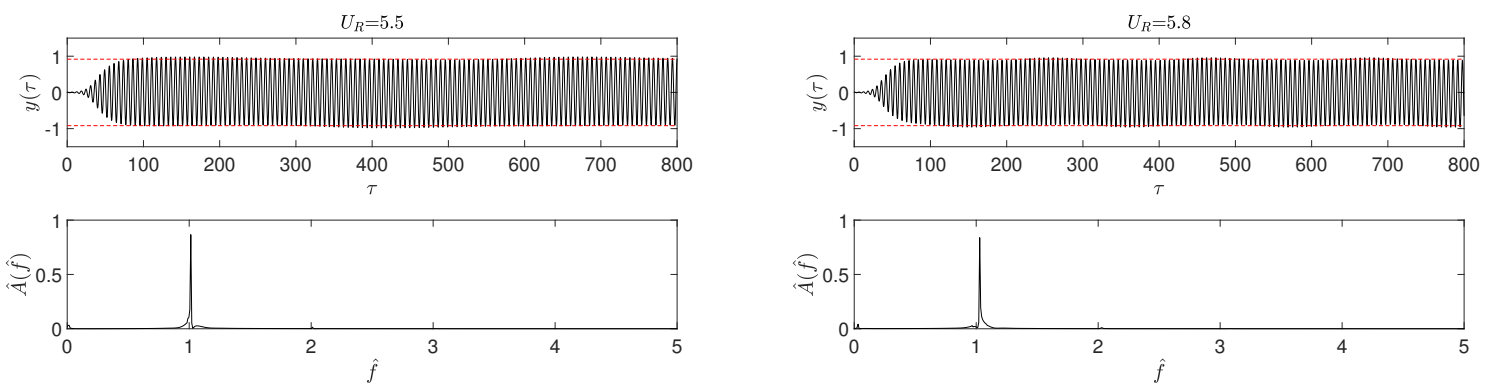

(a) $U_{R}=5,5$.

(b) $U_{R}=5,8$.

Fonte: O autor.

Buscando obter um melhor entendimento nas análises espectrais, foi aplicada a técnica de HHT no domínio tempo-frequência. A Figura 22 apresenta o espectro de HilbertHuang para o caso combinado de VIV e excitação paramétrica $\left(n_{y}=1\right.$ e $\left.\delta_{y}=0,10\right)$. Além disso, com o intuito de melhorar a visualização do espectro de HHT, foi selecionado o intervalo de tempo $350<\tau<650$ ilustrando somente alguns ciclos de oscilação.

Analisando os espectros de Hilbert-Huang da Figura 22, nota-se que a frequência instantânea varia com uma modulação entre $\hat{f} \approx 1$ e $\hat{f} \approx 1,08$. Esse resultado indica que a resposta do cilindro possui uma modulação em frequência pequena, porém com amplitude instantânea $a_{y}^{*} \approx 0,9$. Comparando os espectros das Figuras 22a e 22b, percebe-se que para $U_{R}=5,8$ existe uma modulação em frequência mais acentuada que $U_{R}=5,5$.

Figura 22 - Espectro de Hilbert-Huang $\left(\delta_{y}=0,10\right.$ e $\left.n_{y}=1\right)$
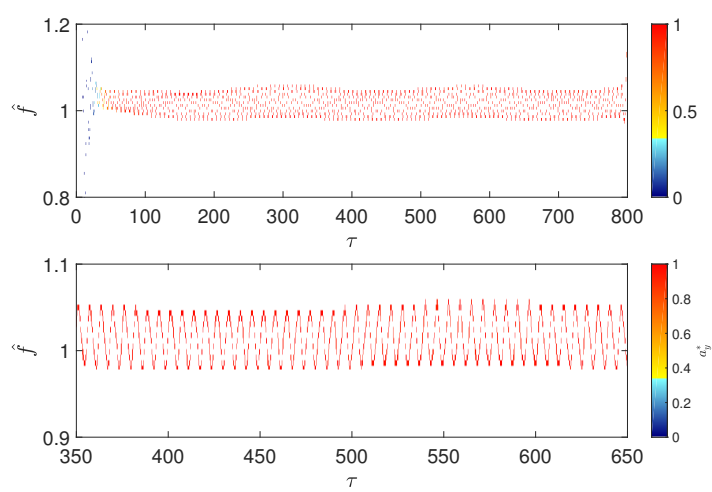

(a) Espectro Hilbert-Huang: $U_{R}=5,5$.
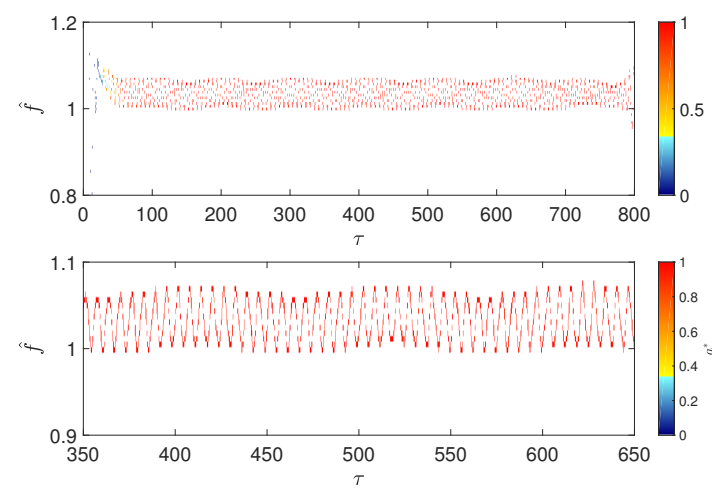

(b) Espectro Hilbert-Huang: $U_{R}=5,8$.

Fonte: $\mathrm{O}$ autor. 


\subsubsection{Resultados considerando $n_{y}=2$}

A Figura 23 ilustra a curva de amplitude característica em função da velocidade reduzida para o caso $n_{y}=2$ com variação da amplitude de excitação $\delta_{y}$ de 0,02 à 0,12. Como é claramente revelado pela Figura 23 a instabilidade paramétrica principal afeta a curva $\hat{A}_{y}\left(U_{R}\right)$ para velocidades reduzidas próximas ao pico de resposta dentro do lockin $\left(5,0 \leq U_{R} \leq 6,5\right)$. Do ponto de vista quantitativo, a amplitude máxima saltou de $\max \left\{\hat{A}_{y}\right\} \approx 0,9$ ("VIV Puro") para $\max \left\{\hat{A}_{y}\right\} \approx 1,3$ (instabilidade paramétrica principal com $\left.\delta_{y}=0,10\right)$, o que representa um ganho de $40 \%$ em amplitude.

Figura 23 - Curvas de respostas da amplitude característica

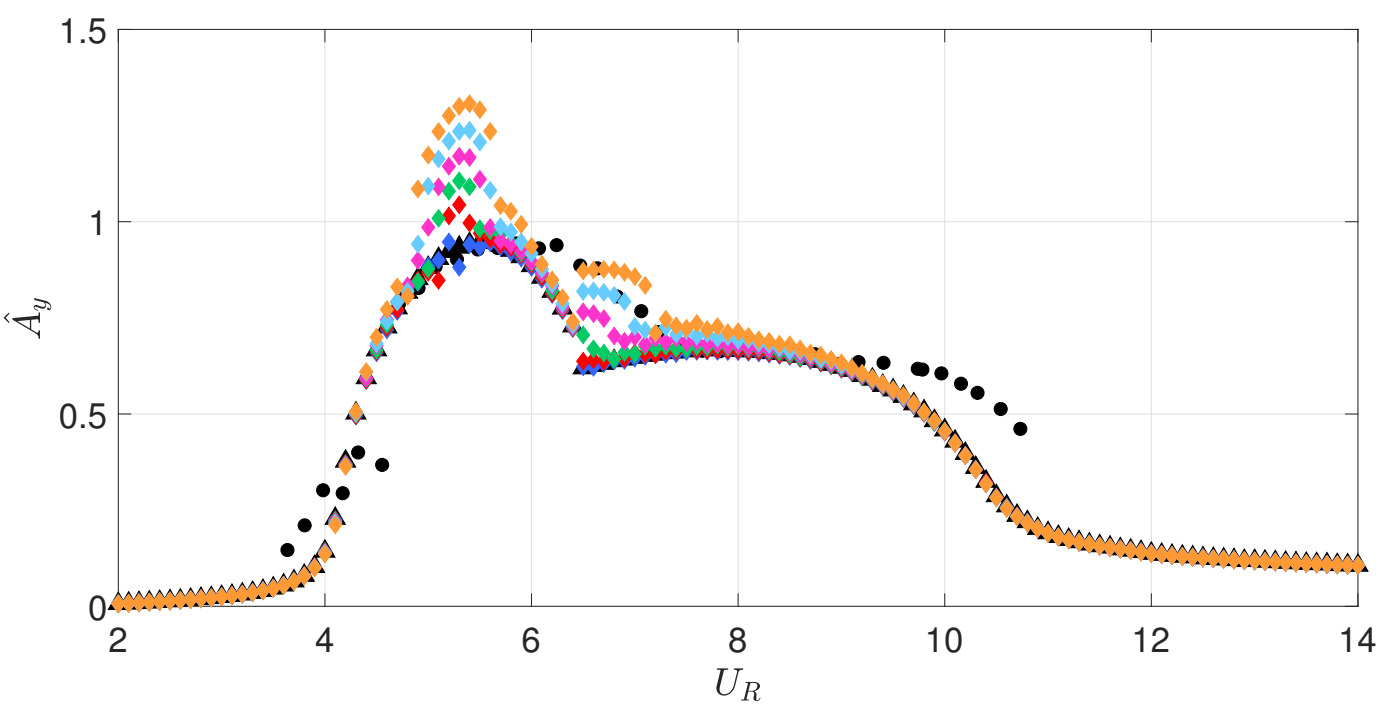

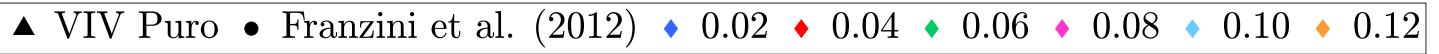

Fonte: O autor.

Considerando os casos de $\delta_{y}=0,02$ e $\delta_{y}=0,04$, para as velocidades reduzidas correspondentes ao ramo de resposta lower-branch que é definido entre $6,5 \leq U_{R} \leq 9,0$, percebe-se que não houve aumento da amplitude característica, que permanece com amplitude aproximadamente constante de $\hat{A}_{y} \approx 0,6$. Ainda analisando a Figura 23, observa-se que para outros valores de $\delta_{y}=0,06$ e $\delta_{y}=0,08$, há um ligeiro aumento no valor de $\max \left\{\hat{A}_{y}\right\} \approx 0,6$ ("VIV Puro") para $\max \left\{\hat{A}_{y}\right\} \approx 0,7\left(\delta_{y}=0,10\right)$.

A Figura 24 apresenta as amplitudes características de oscilação na direção crosswise ao longo do cilindro flexível totalmente submerso. O movimento imposto ao topo do cilindro é dado pela frequência de excitação $\left(f_{t}\right)$, o que induz a variação temporal da rigidez. O foco desta subseção é para o caso $f_{t}: f_{N, 1}=2: 1$, no qual $f_{N, 1}$ corresponde à frequência natural do primeiro modo de vibrar, que indica a instabilidade paramétrica principal com o primeiro modo de vibrar.

Como pode ser visto na Figura 24a para a condição "VIV Puro"para $U_{R}=5,63$, 
o valor máximo da amplitude característica próximo ao meio do vão é $\max \left\{\hat{A}_{y}\right\} \approx 0,6$ enquanto, para o caso combinado com $\Delta N / \bar{N}=50 \%$ apresentado na Figura $24 \mathrm{~b}$ com velocidade reduzida igual a $U_{R}=5,80$, o valor $\max \left\{\hat{A}_{y}\right\} \approx 1,3$, ou seja, houve um aumento de praticamente $100 \%$ na amplitude de oscilação.

Figura 24 - Amplitude característica de oscilação direção na cross-wise

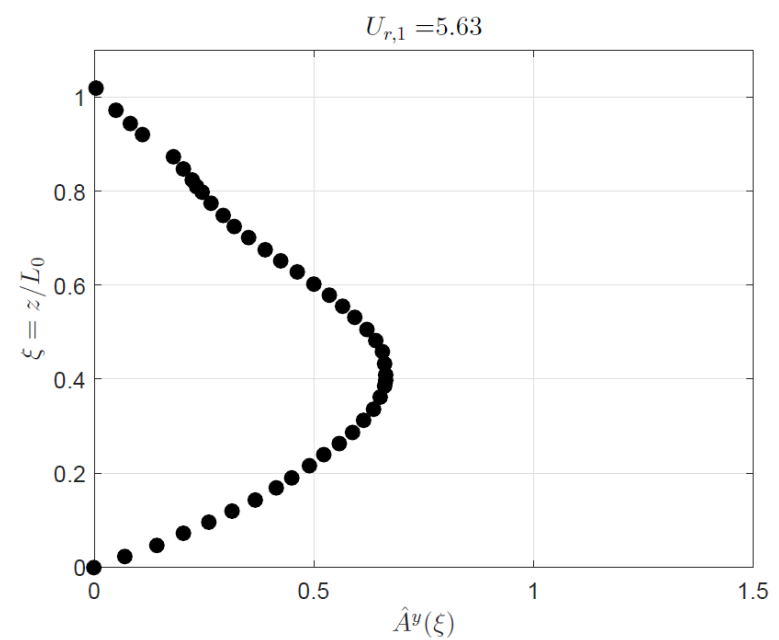

(a) "VIV Puro".

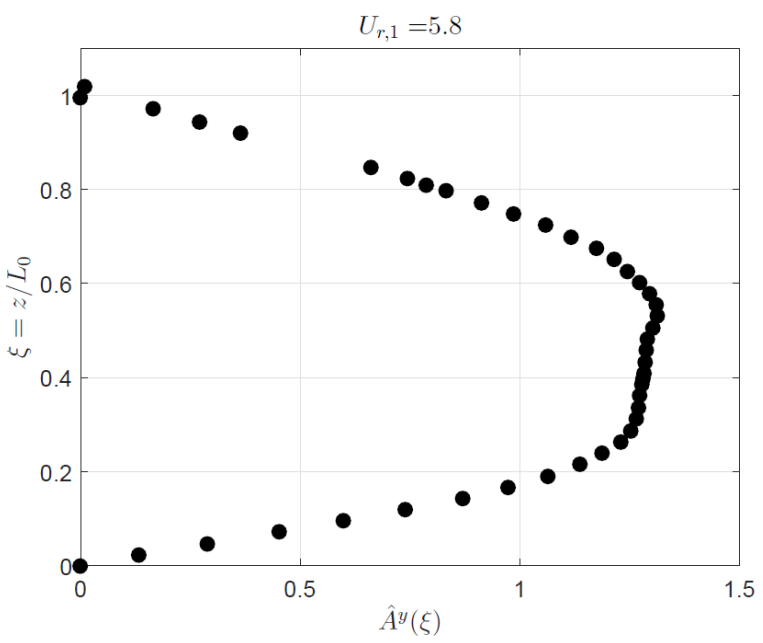

(b) $f_{t}: f_{n, 1}=2: 1$.

Fonte: Extraído de Franzini et al. (2018).

Comparando a curva de amplitude de resposta na direção transversal para o cilindro rígido (ver Figura 23), com as amplitudes características do cilindro flexível na mesma direção presentes na Figura 24, é possível identificar que a excitação paramétrica aumenta ambas as amplitudes de respostas dos cilindros com velocidade reduzidas somente no intervalo de $5,5 \leq U_{R} \leq 6,0$.

As Figuras 25a e 25b mostram as series temporais de deslocamento obtidas a partir do modelo fenomenológico para as mesmas velocidades reduzidas considerando o caso $\delta_{y}=0,10$ e $n_{y}=2$. Analisando ambas figuras, nota-se uma amplificação na amplitude de resposta do cilindro devido à excitação paramétrica na região de máximo lock-in. Para o caso de $U_{R}=5,5$ não existe modulação de amplitude, porém é possível perceber um acréscimo da amplitude característica de oscilação em comparação com a condição "VIV Puro". No que se refere ao espectro de amplitude, nota-se que a frequência $\hat{f}=1$ e possui uma banda estreita.

Já no caso $U_{R}=5,8$ é possível notar a modulação de amplitude e também um acréscimo da amplitude característica de oscilação, além disso observa-se o alargamento da banda espectral com a presença de dois pequenos picos de frequência adimensional próximos da frequência dominante de $\hat{f} \approx 1$. Esse resultado está em concordância qualitativa com os resultados experimentais de Franzini et al. (2018), lembrando que existe uma diferença entre a amplitude de excitação paramétrica dos experimentos e aquela aqui apresentada. 
Figura 25 - Series temporais de resposta do cilindro para o $\operatorname{caso} \delta_{y}=0,10$ e $n_{y}=2$
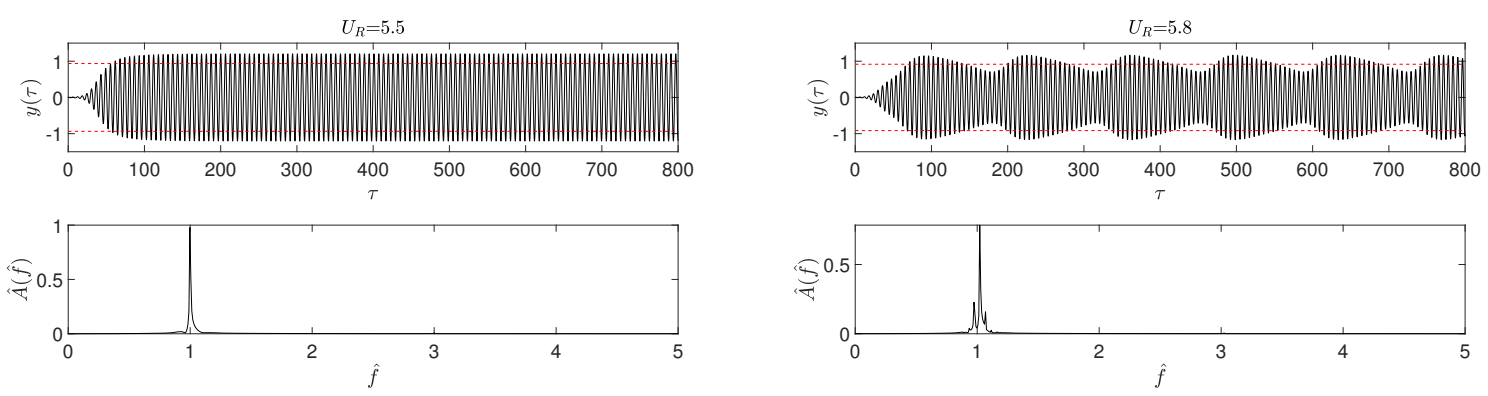

(a) $U_{R}=5,5$.

(b) $U_{R}=5,8$.

Fonte: O autor.

Focando na análise da série temporal de deslocamento do cilindro flexível apresentada na Figura 26, nota-se que, a resposta do cilindro para o caso "VIV Puro"mostrado na Figura 26a, é livre de modulação, possui amplitude constante $\hat{A}_{y}=0,6$ e banda estreita com frequência dominante $\hat{f}=1$. Porém, o resultado para a condição de movimento imposto de frequência $f_{t}: f_{N, 1}=2: 1$ mostrado na Figura 26b, revela que a excitação paramétrica amplifica a amplitude de oscilação, além de apresentar forte modulação em amplitude. Já o espectro de amplitude possui um leve alargamento da banda espectral com a frequência centrada em $\hat{f}=1$.

Figura 26 - Series temporais de resposta do cilindro flexível
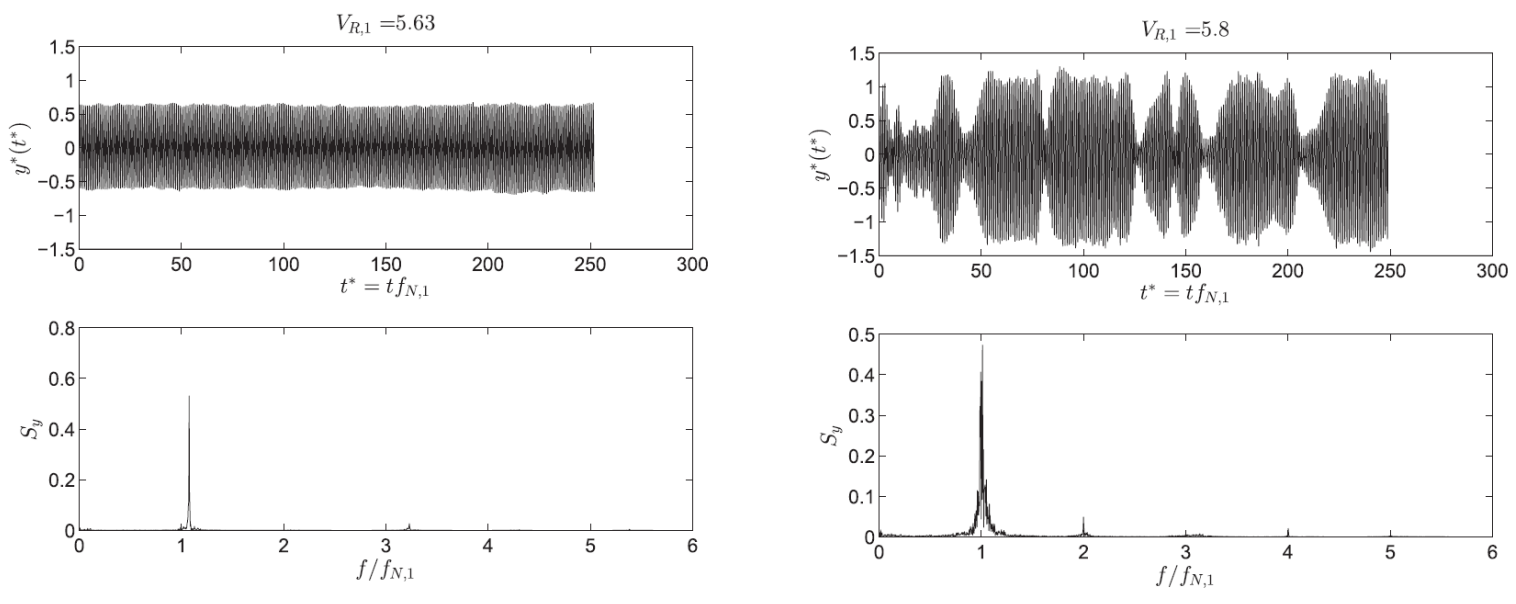

(a) "VIV Puro".

(b) $f_{t}: f_{N, 1}=2: 1$.

Fonte: Extraído de Franzini et al. (2018).

A Figura 27 ilustra as séries temporais de deslocamento do modelo numérico de cilindro flexível estudado por Yuan, Xue e Tang (2018). Como visto na Figura 27a, a resposta para o caso "VIV Puro"também possui amplitude constante $\hat{A}_{y}=0,6 \mathrm{em}$ concordância com o que já foi apresentado na análise da Figura 26a. O espectro de 
amplitude também é consistente com o apresentado no modelo experimental, tendo a frequência dominante $\hat{f}=1$ e banda estreita. Já a Figura $27 \mathrm{~b}$ com $\Delta N / \bar{N}=10 \%$ revela que, a instabilidade paramétrica principal promove um aumento da amplitude característica de $\hat{A_{y}}=0,6$ para $\hat{A_{y}}=1,2$ similar ao observado no experimento, porém a forte modulação não foi obtida no modelo numérico. Outra contribuição presente nessa figura é o espectro de amplitude que possui frequência dominante $\hat{f}=1$, banda estreita e pequenas parcelas em $\hat{f}=2$ e $\hat{f}=3$.

Figura 27 - Series temporais de resposta do cilindro flexível
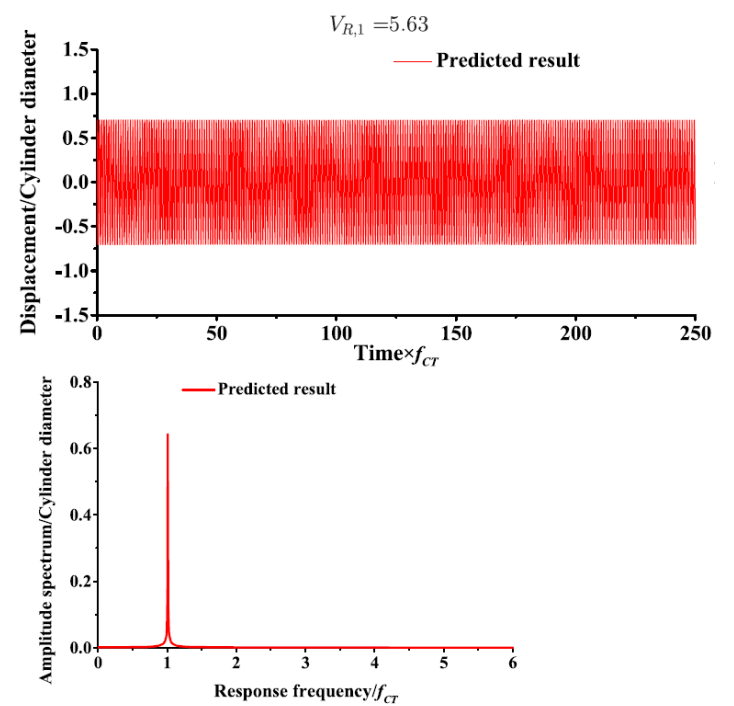

(a) "VIV Puro".
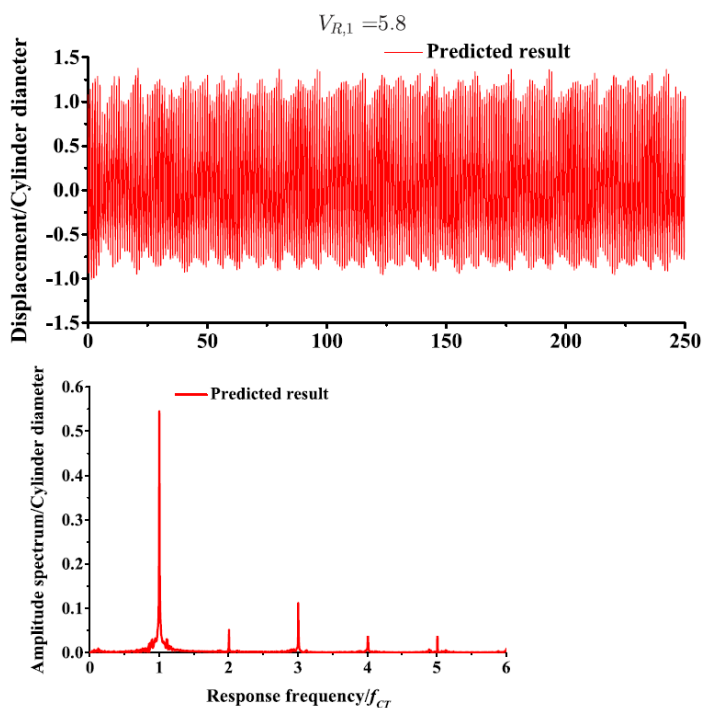

(b) $f_{t}: f_{N, 1}=2: 1$.

Fonte: Adaptado de Yuan, Xue e Tang (2018).

É interessante notar que os resultados numéricos utilizando o modelo fenomenológico (ver Figura 25), também possuem semelhança qualitativa com àqueles vistos na Figura 27. Comparando ambas figuras percebe-se que a amplitude de resposta para o caso combinado de VIV e excitação paramétrica é maior do que no caso "VIV Puro", em que pese as comparações feitas com o modelo numérico de cilindro flexível do trabalho Yuan, Xue e Tang (2018) não foram calibradas da mesma maneira que o modelo fenomenológico abordado nesta pesquisa.

A Figura 28 apresenta o espectro de Hilbert-Huang para o caso $\delta_{y}=0,10$ e $n_{y}=2$, nas velocidades reduzidas de $U_{R}=5,5$ e 5,8. Conforme visto na Figura 28a, é possível observar que o traço existente no espectro não possui modulação ao redor da frequência instantânea $\hat{f} \approx 1$, e a amplitude instantânea $a_{y}^{*}>0,8$. Na Figura 28b, o espectro de Hilbert-Huang revela duas modulações importantes de frequência instantânea, uma lenta e uma rápida, o que pode estar relacionado com a frequência de excitação imposta no problema.

Agora, considerando os valores de velocidades reduzidas $U_{R}$ maiores do que 8,0 (o 
Figura 28 - Espectro de Hilbert-Huang $\left(\delta_{y}=0,10\right.$ e $\left.n_{y}=2\right)$
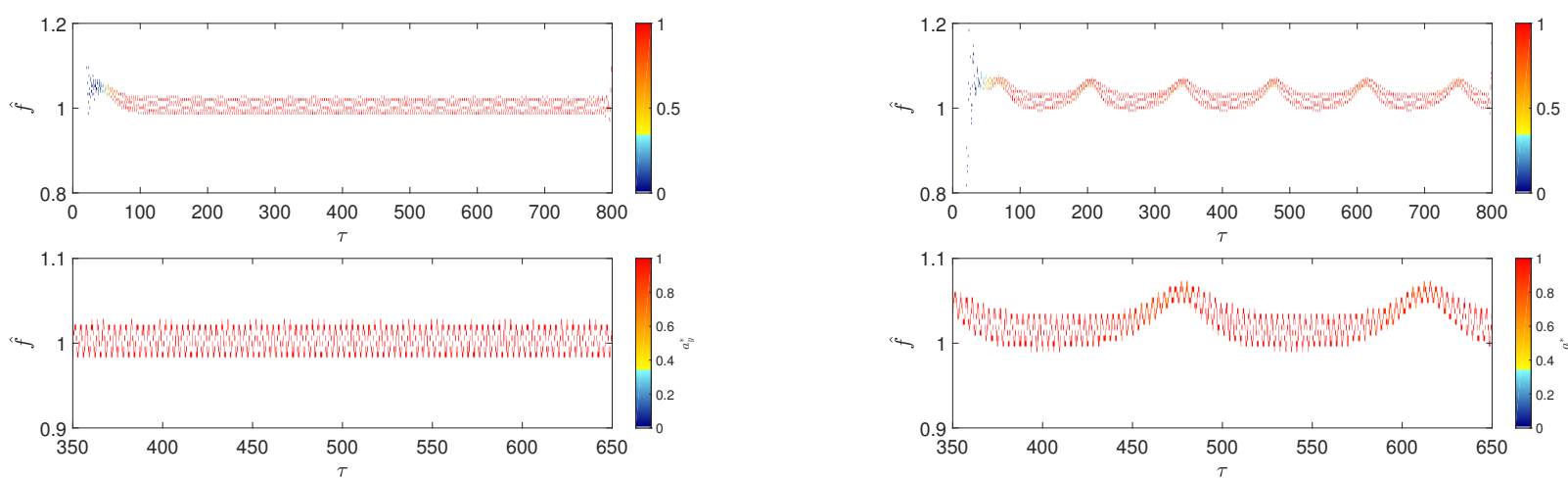

(a) Espectro de Hilbert-Huang: $U_{R}=5,5$.

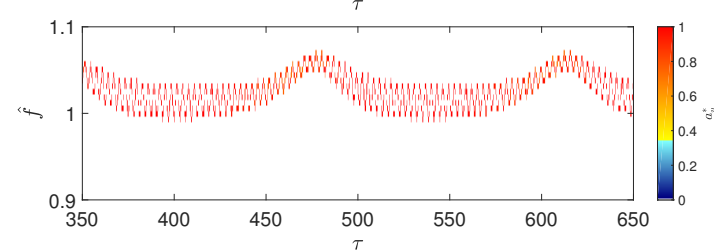

(b) Espectro de Hilbert-Huang: $U_{R}=5,8$.

Fonte: O autor.

que corresponde ao final do ramo lower branch) é possível perceber que não há aumento de amplitude característica para o VIV-1GL (ver Figura 23). Como pode ser visto na Figura 29, analisando as séries temporais de deslocamento do cilindro para nas velocidades reduzidas de $U_{R}=10,4$ e 10,8 considerando a condição $\delta_{y}=0,10$ e $n_{y}=2$, a amplitude de deslocamento não é afetada pela excitação paramétrica. De fato, tanto a Figura 29a quanto a Figura 29b possuem suas respectivas amplitudes de deslocamento iguais àquela na condição "VIV Puro". Outro aspecto interessante é o surgimento de uma componente de frequência próxima de $\hat{f} \approx 0,5$ em ambas velocidades reduzidas.

Figura 29 - Series temporais de resposta do cilindro para o caso $\delta_{y}=0,10$ e $n_{y}=2$
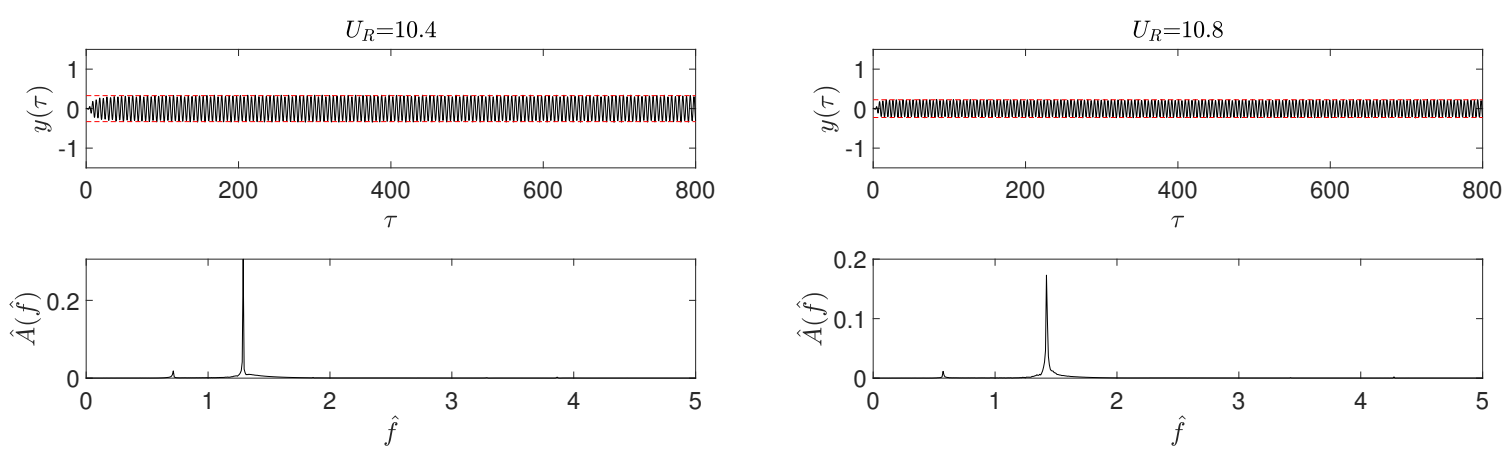

(a) $U_{R}=10,4$.

(b) $U_{R}=10,8$.

Fonte: O autor.

Na Figura 30 é possível observar as séries temporais de amplitudes modais do trabalho de Franzini et al. (2018) associadas com o primeiro modo de vibrar na direção cross-wise e $U_{R} \approx 13,7$. Como mostra a Figura 30a, a condição "VIV Puro"possui modulação em amplitude e tem o espectro de amplitude com frequência dominante próxima de $\hat{f} \approx 2,5$. Já a série temporal mostrada na Figura 30b apresenta uma forte modulação em amplitude e o espectro de amplitude tem uma distribuição espectral mais extensa. Tais resultados 
indicam que a resposta do cilindro flexível difere daquela mostrada para o VIV-1GL, pois é possível notar que a resposta do cilindro flexível é mais influenciada pelo movimento imposto $f_{t}: f_{N, 1}=2: 1$.

Figura 30 - Series temporais de resposta do cilindro flexível
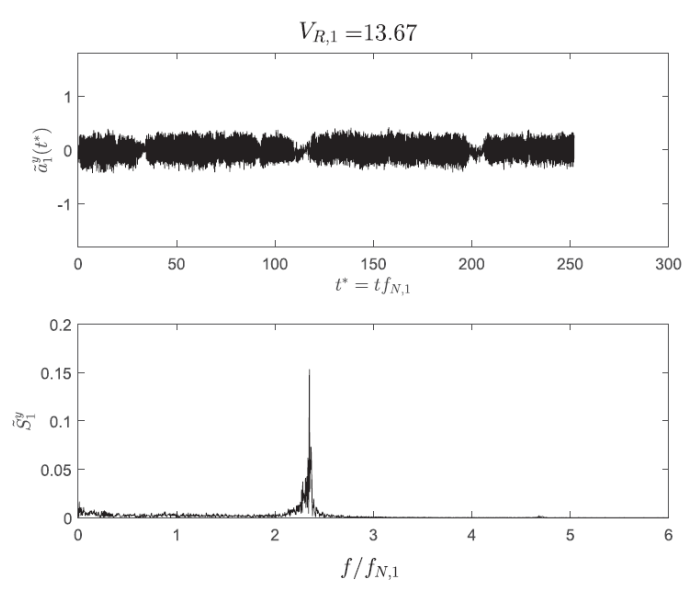

(a) "VIV Puro".
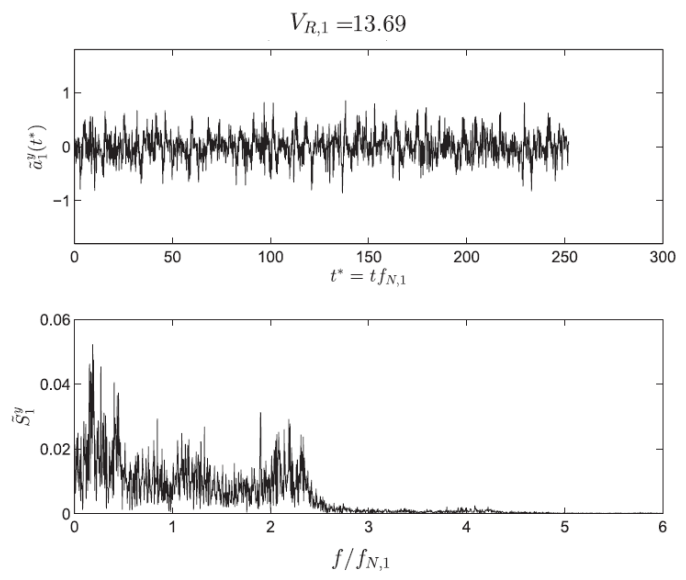

(b) $f_{t}: f_{n, 1}=2: 1$.

Fonte: Extraído de Franzini et al. (2018).

Na Figura 31 é possível observar o espectro de Hilbert-Huang para o caso $n_{y}=2$ nas velocidades reduzidas de $U_{R}=10,4$ e 10,8 e revela dois traços de frequência. O traço de maior frequência possui componente $\hat{f} \approx 1,5$, com uma baixa modulação de amplitude instantânea definida no intervalo de $0,4 \leq a_{y}^{*} \leq 0,5$. Já o traço de menor frequência, por sua vez, mostra uma componente de frequência $\hat{f} \approx 0,75$, que apresenta uma pequena energia associada comparada àquela anterior tendo a sua amplitude instantânea $0 \leq a_{y}^{*} \leq 0,1$. Esse fato mostra que a presença da excitação paramétrica continua atuando no sistema, mesmo para maiores valores de velocidades reduzidas, porém sem alterar o valor máximo de amplitude característica de oscilação quando comparado com o caso "VIV Puro".

A seguir, será ilustrada a influência da variação de $n_{y}$ e $\delta_{y}$ na amplitude característica de oscilação como função de $U_{R}\left(\hat{A}_{y}\left(n_{y}, U_{R}\right)\right)$ na forma de mapas de cores, como mostra a Figura 32, considerando $\delta_{y}=0.10$ para a condição de VIV-1GL. No mapa de cores, considera-se $1 \leq n_{y} \leq 3$ e $2 \leq U_{R} \leq 14$ com uma malha de dimensão $300 \times 300$. A mesma figura mostra, ainda, séries temporais $y(\tau)$ associadas a cada ponto no plano de $\operatorname{parâmetros}\left(n_{y}, U_{R}\right)$.

A Figura 32 indica que existem mudanças na $\hat{A}_{y}\left(n_{y}, U_{R}\right)$ (quando comparado ao caso "VIV Puro") somente no intervalo de $1,9<n_{y} \leq 2,2 \times 4,5<U_{R} \leq 6,0$, próximo à excitação paramétrica principal e ao pico da região de lock-in. Nota-se que há uma pequena amplificação da resposta no ramo lower branch $\left(7 \leq U_{R} \leq 9\right)$ para $1,9<n_{y} \leq 2,2$. Os resultados para $n_{y}=1$ praticamente não sofrem amplificações da resposta na curva de $A_{y}$ 
Figura 31 - Espectro de Hilbert-Huang caso-2 $\left(\delta_{y}=0,10\right.$ e $\left.n_{y}=2\right)$
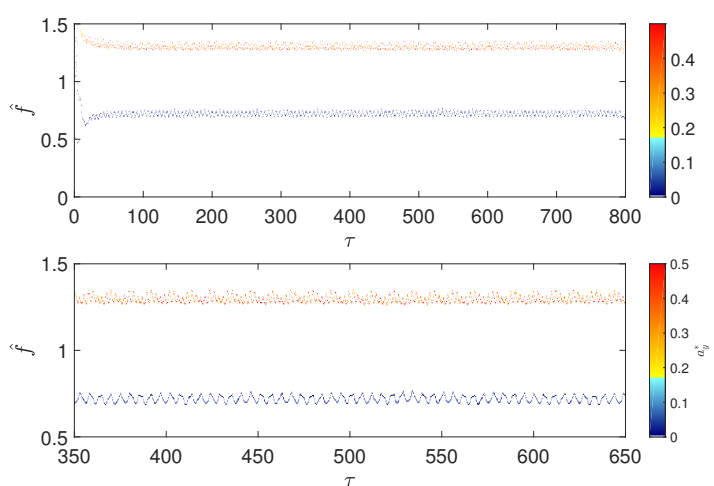

(a) Espectro de Hilbert-Huang: $U_{R}=10,4$.
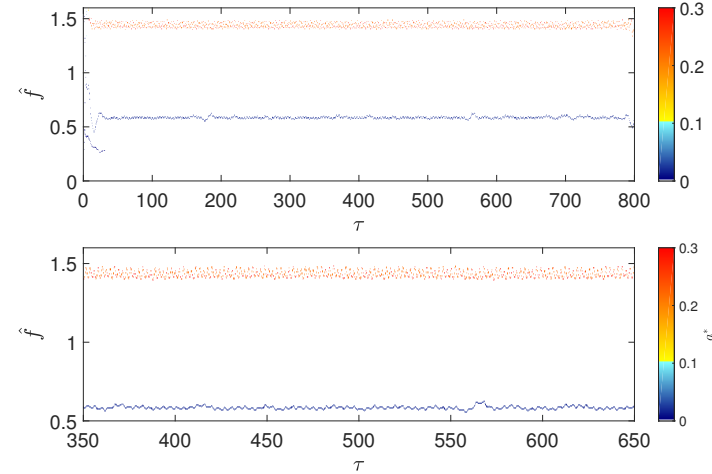

(b) Espectro de Hilbert-Huang: $U_{R}=10,8$.

Fonte: $\mathrm{O}$ autor.

para toda a faixa de velocidades reduzidas. Embora no trabalho de Franzini et al. (2015) discutido na Subseção 4.1, os autores destacam um aumento na amplitude máxima de oscilação na direção cross-wise de $\max \left\{\hat{A}_{y}\right\} \approx 0,7$ na condição de "VIV Puro"para máximo de $\max \left\{\hat{A}_{y}\right\} \approx 1,0$ na região de lock-in.

Figura 32 - Mapa de amplitude para o caso de $\delta_{y}=0,10$
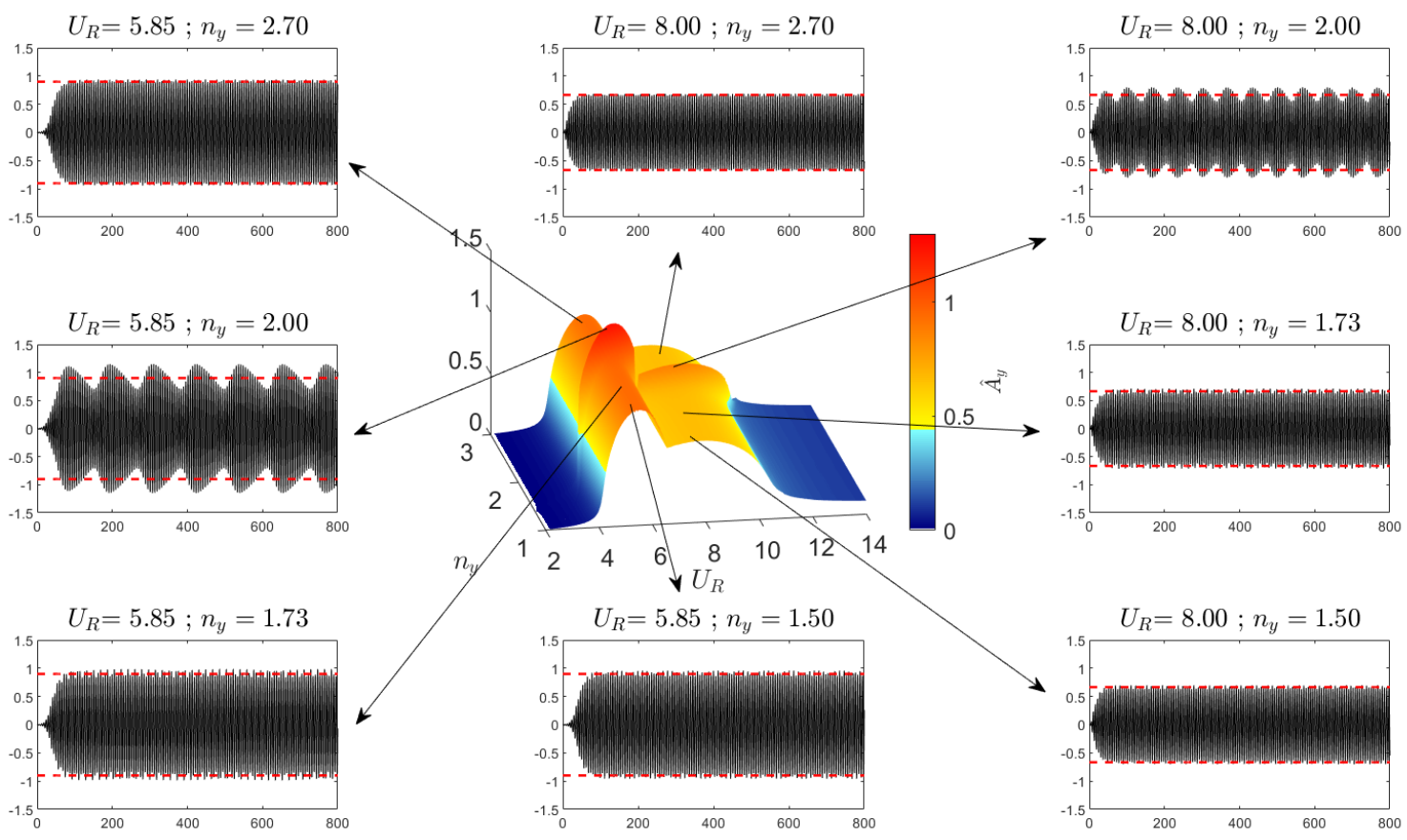

Fonte: $\mathrm{O}$ autor.

Em sequência, na Subseção 4.1.3 discutem-se os resultados numéricos dos coeficientes de força longitudinal médio $\overline{C_{x}}$, coeficiente total de força transversal $C_{y, r . m . s} \mathrm{e}$ o coeficiente de força longitudinal $C_{x, r . m . s}$, são apresentados como função da velocidade 
reduzida $U_{R}$.

\subsubsection{Coeficientes de força hidrodinâmica VIV-1GL}

A Figura 33 mostra os estudos numéricos-experimentais, variando a amplitude de excitação paramétrica $\delta_{y}$ em $\overline{C_{x}}\left(U_{R}\right), C_{y, r . m . s}\left(U_{R}\right)$ e o $C_{x, r . m . s}\left(U_{R}\right)$. Enfatiza-se que os resultados experimentais apresentados em Franzini et al. (2012) foram feitos sob a condição "VIV Puro". Apesar disso, é possível notar que os resultados numéricos possuem um comportamento similar ao experimental. Como pode ser visto na Figura 33, os coeficientes de força são amplificados para 4,5< $U_{R} \leq 6,0$ quando a amplitude de excitação paramétrica é aumentada. A menos do conhecimento do autor, não foram encontradas curvas de coeficientes de força para a excitação paramétrica combinada ao VIV, os resultados aqui apresentados são uma contribuição para a literatura.

Figura 33 - Influência de $\delta_{y}$ nos coeficientes de força em relação à velocidade reduzida $U_{R}$

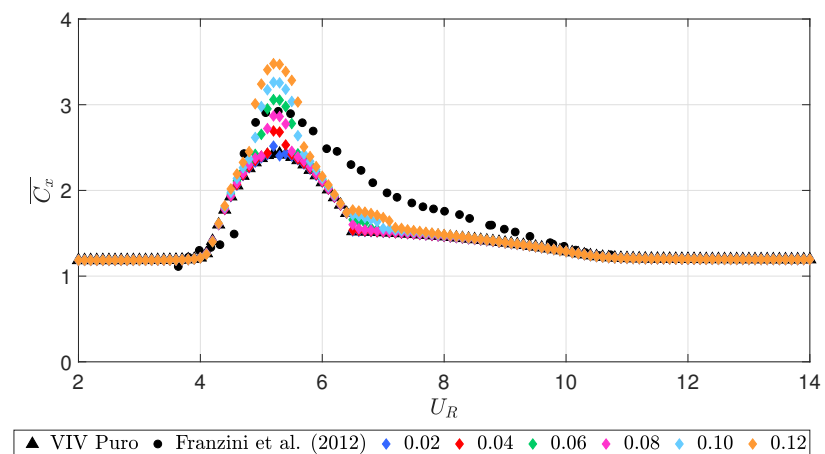

(a) $\overline{C_{x}}\left(U_{R}\right)$.

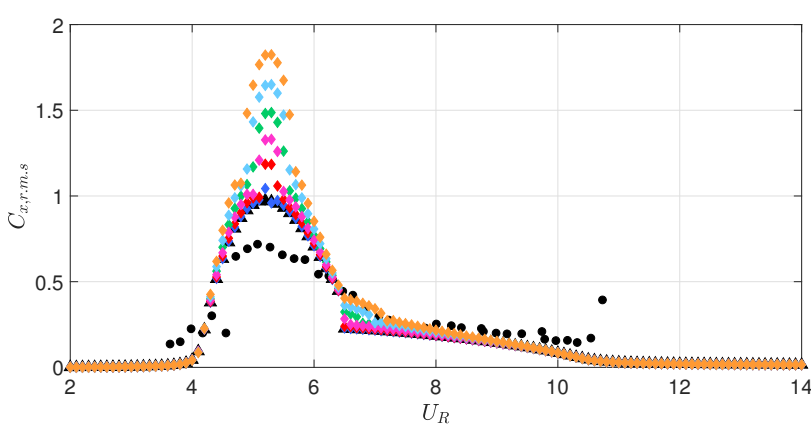

$\Delta$ VIV Puro • Franzini et al. $(2012) \bullet 0.02 \bullet 0.04 \bullet 0.06 \bullet 0.08 \bullet 0.10 \bullet 0.12$

(b) $C_{x, r . m . s}\left(U_{R}\right)$.

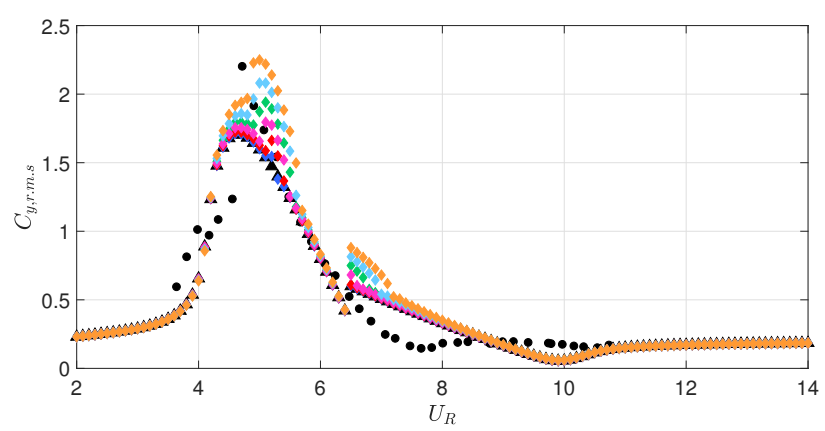

$\mathbf{\Lambda}$ VIV Puro $\bullet$ Franzini et al. $(2012) \bullet 0.02 \bullet 0.04 \bullet 0.06 \bullet 0.08 \bullet 0.10 \bullet 0.12$

(c) $C_{y, r . m . s}\left(U_{R}\right)$.

Fonte: $\mathrm{O}$ autor.

Agora, na Figura 34 são vistos os coeficientes $\overline{C_{x}}\left(n_{y}, U_{R}\right)$ e $C_{y, r . m . s}\left(n_{y}, U_{R}\right)$, na forma de mapa de cores para condição VIV-1GL considerando $\delta_{y}=0,10$. Assim como feito na Subseção 4.1.2, a discretização dos mapas segue o apresentado para $\hat{A}_{y}\left(n_{y}, U_{R}\right)$ (ver 
Figura 32). De acordo com a Figura 34a, é interessante notar que existe um acréscimo nos valores do $\overline{C_{x}}$ com o aumento da frequência de excitação paramétrica no intervalo de $1,8<n_{y} \leq 2,1 \times 4,2<U_{R} \leq 6,2$, correspondendo ao upper-branch. O valor máximo de $\overline{C_{x}}$ é $\max \left\{\overline{C_{x}}\right\}=3,20$, consideravelmente superior a $\max \left\{\overline{C_{x}}\right\}=2,40$ ("VIV Puro").

Como pode ser notado na Figura $34 \mathrm{~b}$, o aumento do $C_{y, r . m . s}$ é mais evidente para o mesmo intervalo de variação de $n_{y} \times U_{R}\left(1,8<n_{y} \leq 2,1 \times 4,2<U_{R} \leq 6,2\right)$ àquele observado para $\overline{C_{x}}$. Nota-se que o valor máximo de $C_{y, r . m . s}$ é $\max \left\{C_{y, r . m . s}\right\}=2,4$, significativamente maior que $\max \left\{C_{y, r . m . s}\right\}=1,68$ ("VIV Puro"). Além disso, é importante ressaltar que a região do plano de parâmetros $\left(n_{y} ; U_{R}\right)$ onde os valores de $C_{y, r . m . s}$ são maiores que 1,6 concentram-se na faixa de velocidade reduzidas $4,0<U_{R} \leq 5,5$. As outras regiões, que são representadas pela cor azul escuro possuem valores de $C_{y, r . m . s}$ abaixo de 0,8 .

Figura 34 - Mapa dos coeficientes de força para $\delta_{y}=0,10$

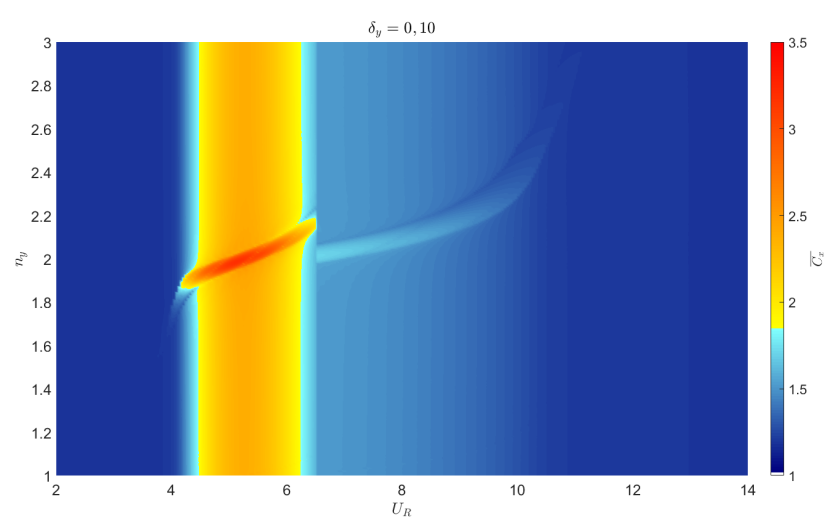

(a) $\overline{C_{x}}\left(n_{y}, U_{R}\right)$.

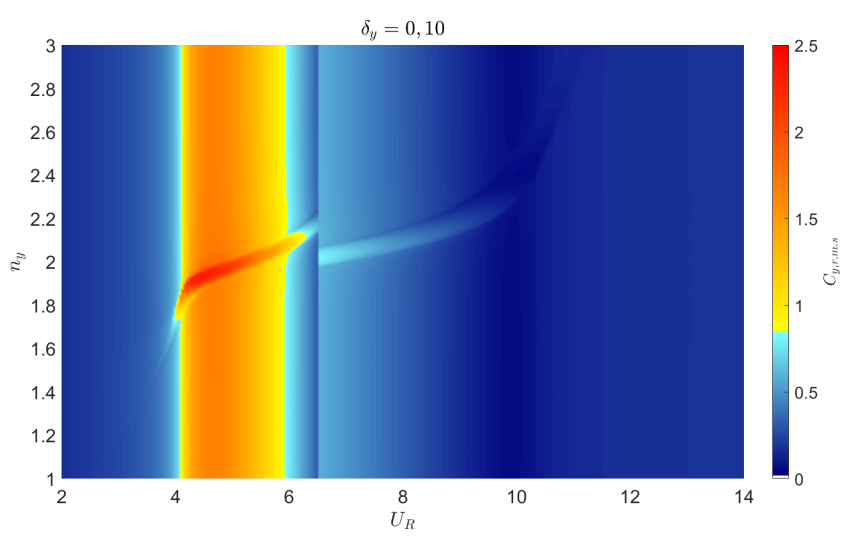

(b) $C_{y, r . m . s}\left(n_{y}, U_{R}\right)$.

Fonte: O autor.

Por fim, são mostrados os mapas isométricos dos coeficientes $\overline{C_{x}}\left(n_{y}, U_{R}\right)$ e $C_{y, r . m . s}\left(n_{y}, U_{R}\right)$ na Figura 35. Esse resultado é o mesmo que o obtido na Figura 34, porém visto de outra maneira. Aqui, os mapas são mostrados em conjunto com as séries temporais $C_{x}(\tau)$ e $C_{y}(\tau)$ para algumas combinações no plano $\left(n_{y}, U_{R}\right)$. O leitor pode notar que as séries temporais de $C_{x}(\tau)$ e $C_{y}(\tau)$ relacionadas aos pontos $\left(n_{y}, U_{R}\right)=(2,00,5,10)$ e $\left(n_{y}, U_{R}\right)=(2,00,4,50)$, respectivamente, apresentam valores máximos significativamente maiores àqueles obtidos para condição "VIV Puro". Já as combinações $\left(n_{y}, U_{R}\right)=(2,00,7,50)$ e $\left(n_{y}, U_{R}\right)=(2,00,7,30)$, que dizem respeito ao lower-branch, possuem modulações em amplitude de oscilação dos coeficientes de força $C_{x}(\tau)$ e $C_{y}(\tau)$, porém com valores máximos inferiores ao máximo obtidos no upper-branch. 
Figura 35 - Mapa dos coeficientes de força $\overline{C_{x}}$ e $C y_{r . m . s}$ para o caso de $\delta_{y}=0,10$
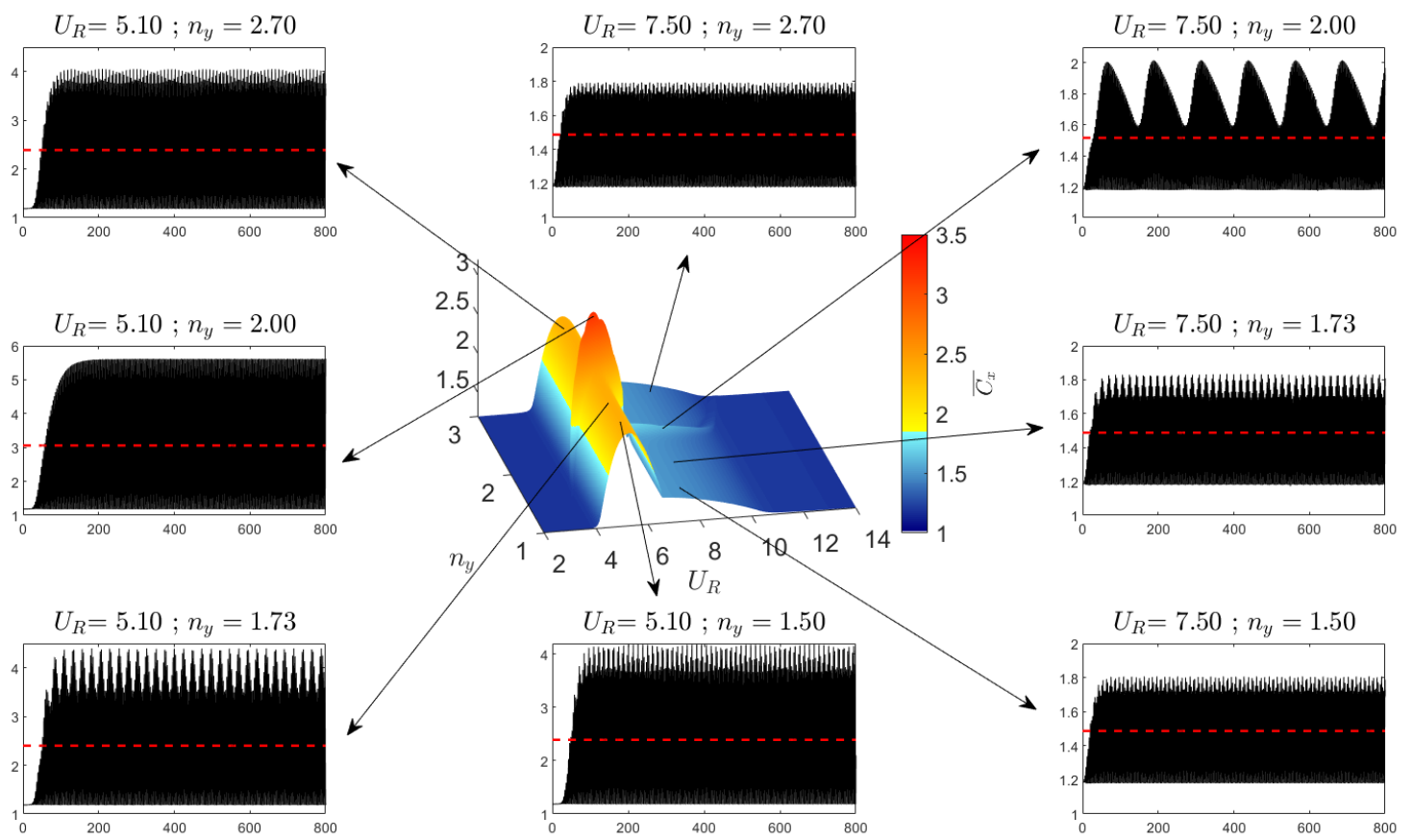

(a) $\overline{C_{x}}\left(n_{y}, U_{R}\right)$ e resposta do $C_{x}(\tau)$.
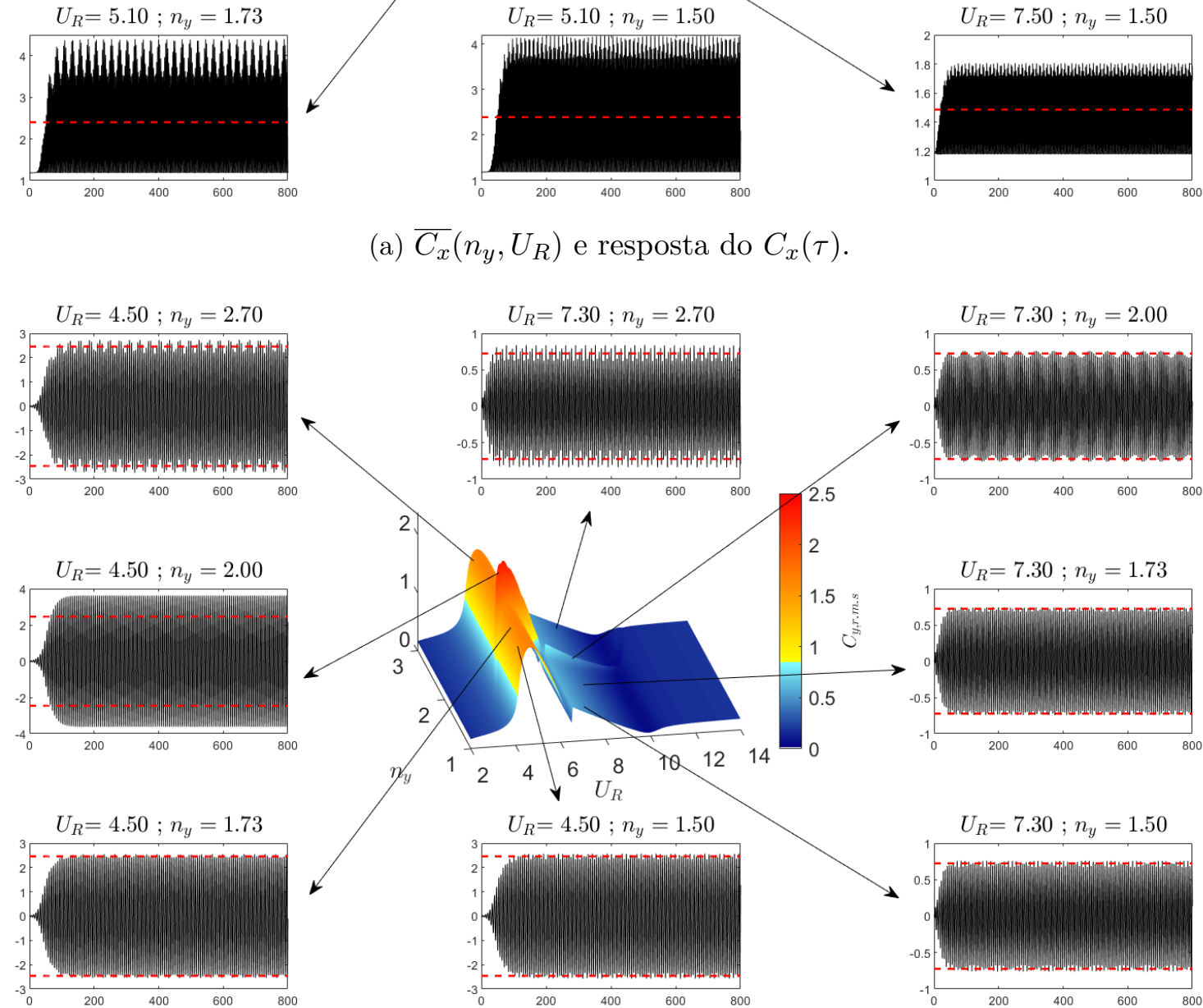

(b) $C_{y, r . m . s}\left(n_{y}, U_{R}\right)$ e resposta do $C_{y}(\tau)$.

Fonte: $\mathrm{O}$ autor. 


\subsubsection{Seção de Poincaré}

Esta Subseção apresenta os pontos nas seções de Poincaré, para o casos "VIV Puro"e VIV combinado ao fenômeno de excitação paramétrica, por sua vez, estão ilustrados nas Figuras 36 e 37. Os pontos em vermelho foram tomados na seção estroboscópica, definida com o período igual ao da excitação paramétrica. Observando as seções é possível perceber um comportamento típico de sistemas quase-periódicos.

Figura 36 - Mapa de Poincaré: VIV Puro

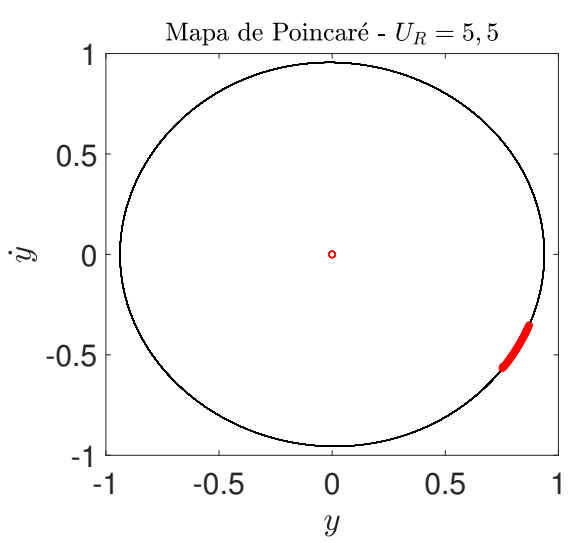

(a) $U_{R}=5,5$.

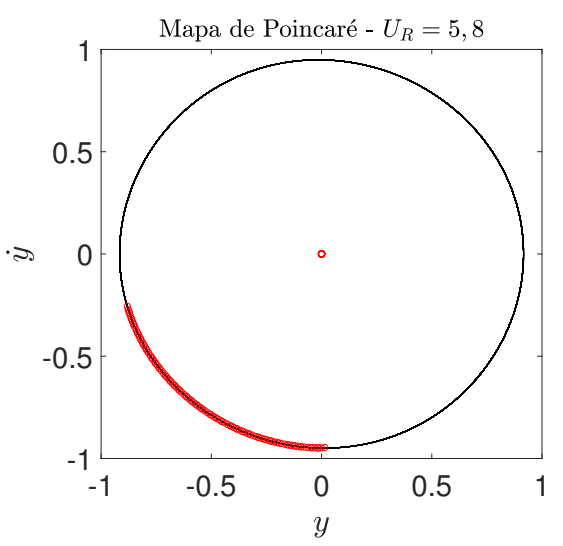

(b) $U_{R}=5,8$.

Fonte: O autor.

Figura 37 - Mapa de Poincaré: $\delta_{y}=0.10$ e $n_{y}=2$

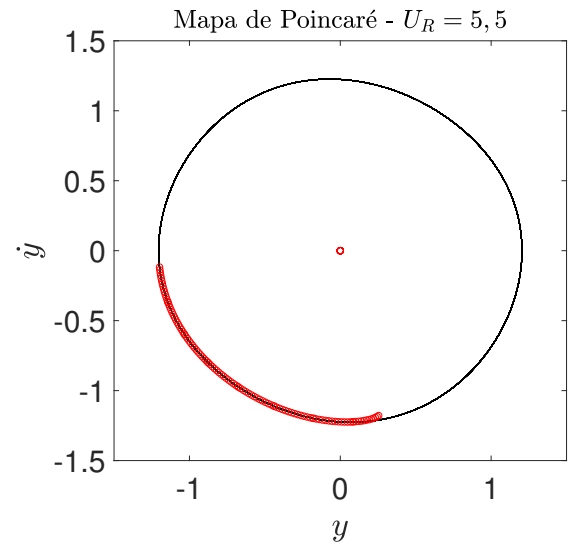

(a) $U_{R}=5,5$.

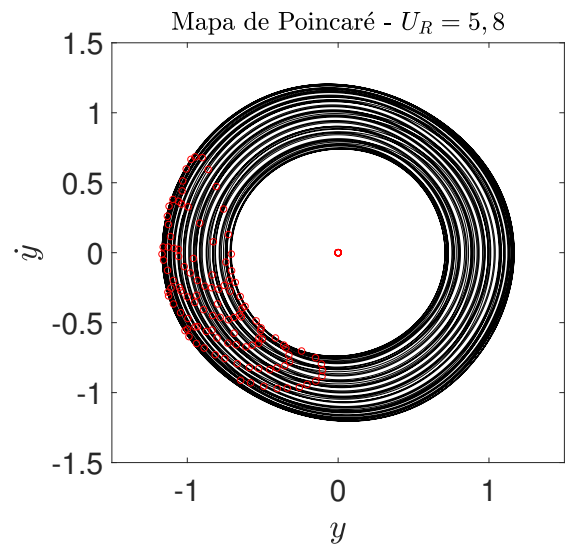

(b) $U_{R}=5,8$.

Fonte: O autor.

\subsection{5 Órbitas no espaço de fase}

Esta Subseção tem como objetivo apresentar as órbitas no espaço de fase no $\mathbb{R}^{4}$. O estudo das trajetórias no espaço de fase estabelece uma importante técnica na definição do comportamento de sistemas dinâmicos. Para construir o espaço de fases é necessário 
conhecer as variáveis de estado que governam o sistema. Essas variáveis são representadas pela posição do cilindro $y(\tau)$, velocidade do cilindro $\dot{y}(\tau)$, posição da esteira $q_{y}(\tau)$ e velocidade da esteira $\dot{q}_{y}(\tau)$.

Com intuito de visualizar o espaço 4D em um gráfico 3D, projeta-se cada ponto do espaço de fase 4D no espaço de fase 3D (com três variáveis de estado: $y(\tau), \dot{y}(\tau)$ e $q_{y}(\tau)$ ), sendo a variável de estado $\dot{q}_{y}(\tau)$ representada na região colorida. As Figuras 38 e 39, mostram as trajetórias da soluções para os casos "VIV Puro"e para o caso combinado de excitação paramétrica e VIV, com suas respectivas projeções nos planos de fase.

A Figura 38 apresenta como a trajetória se comporta no espaço de fase para o caso "VIV Puro", considerando as velocidades reduzidas $U_{R}=5,5$ e $U_{R}=5,8$. Comparando as Figuras 38a e 38b, é possível notar que ambas são órbitas periódicas, cujas as trajetórias são curvas fechadas.

Figura 38 - Órbitas no espaço de fase caso: VIV Puro

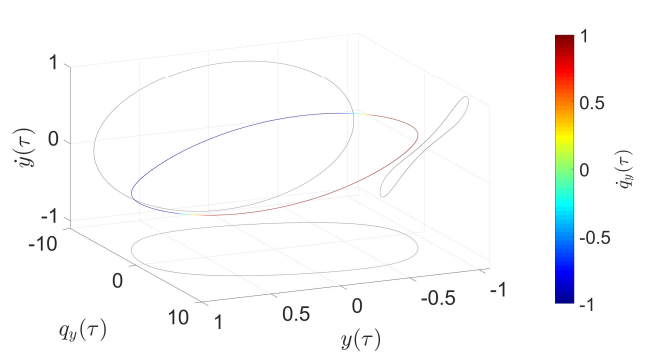

(a) $U_{R}=5,5$.

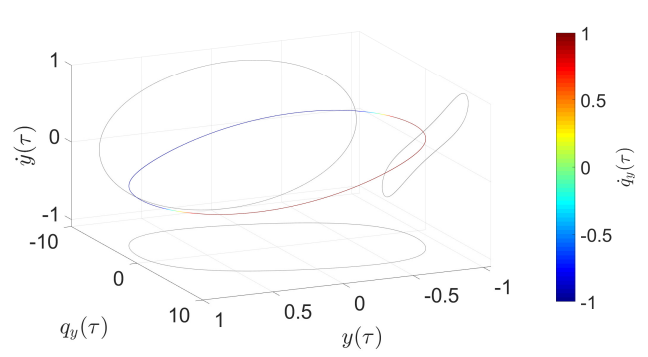

(b) $U_{R}=5,8$.

Fonte: O autor.

Já a Figura 39 mostra como a trajetória se comporta no espaço de fase para o caso combinado de VIV e excitação paramétrica, considerando as velocidades reduzidas $U_{R}=5,5$ e $U_{R}=5,8$. Comparando as Figuras 39a e 39b, é possível notar que as órbitas ocupam uma faixa mais larga no espaço de fase em $U_{R}=5,8$ cujas as trajetórias definem um toro, indicando soluções quase-periódicas.

Figura 39 - Órbitas no espaço de fase caso: $\delta_{y}=0.10$ e $n_{y}=2$

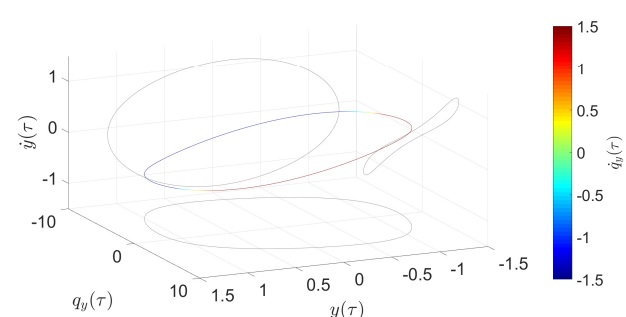

(a) $U_{R}=5,5$.

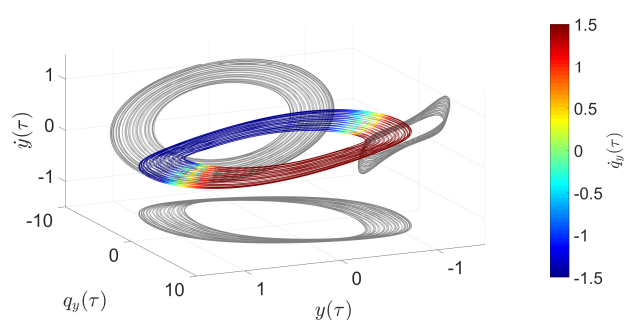

(b) $U_{R}=5,8$.

Fonte: O autor. 


\subsection{Resultados numéricos VIV-2GL}

Nesta Seção, o foco é analisar o comportamento do cilindro rígido montado em base elástica livre para oscilar nas duas direções transversal e longitudinal ao escoamento (VIV-2GL). Os resultados numéricos para a condição VIV-2GL, foram obtidos utilizando o modelo fenomenológico de Franzini e Bunzel (2018). A seguir, são apresentados as curvas de amplitude característica do cilindro nas duas direções em função da velocidade reduzida, variando as amplitudes de excitação paramétrica $\delta_{y}=\delta_{x}$, séries temporais de deslocamento do cilindro $y(\tau)$ e $x(\tau)$, coeficientes de força, $\overline{C_{x}}\left(U_{R}\right), C_{y, r . m . s}\left(U_{R}\right)$ e o $C_{x, r . m . s}\left(U_{R}\right)$.

Assim como foi feito na Seção 4.1, as amplitudes características do cilindro para o caso "VIV Puro", também serão mostradas nas séries temporais $y(\tau)$ considerando o problema combinado de VIV e excitação paramétrica por linhas tracejadas em vermelho.

\subsubsection{Resultados considerando $n_{y}=n_{x}=1$}

O foco desta subseção é analisar os estudos numéricos para a condição VIV-2GL, considerando as frequências de excitação paramétrica $n_{y}=n_{x}=1$. As Figuras 40 e 41 mostram as amplitudes características de oscilação $\hat{A}_{y}\left(U_{R}\right)$ e $\hat{A}_{x}\left(U_{R}\right)$. De acordo com a Figura 40, nota-se que o efeito combinado de VIV e excitação paramétrica não afeta a resposta do cilindro na direção transversal ao escoamento. Com isso, comparando esse resultado com aquele obtido para o mesmo valor de frequência de excitação e para condição VIV-1GL (ver Figura 18), percebe-se que o VIV prevalece sobre a excitação paramétrica.

Figura 40 - Curvas de respostas da amplitude característica na direção cross-wise

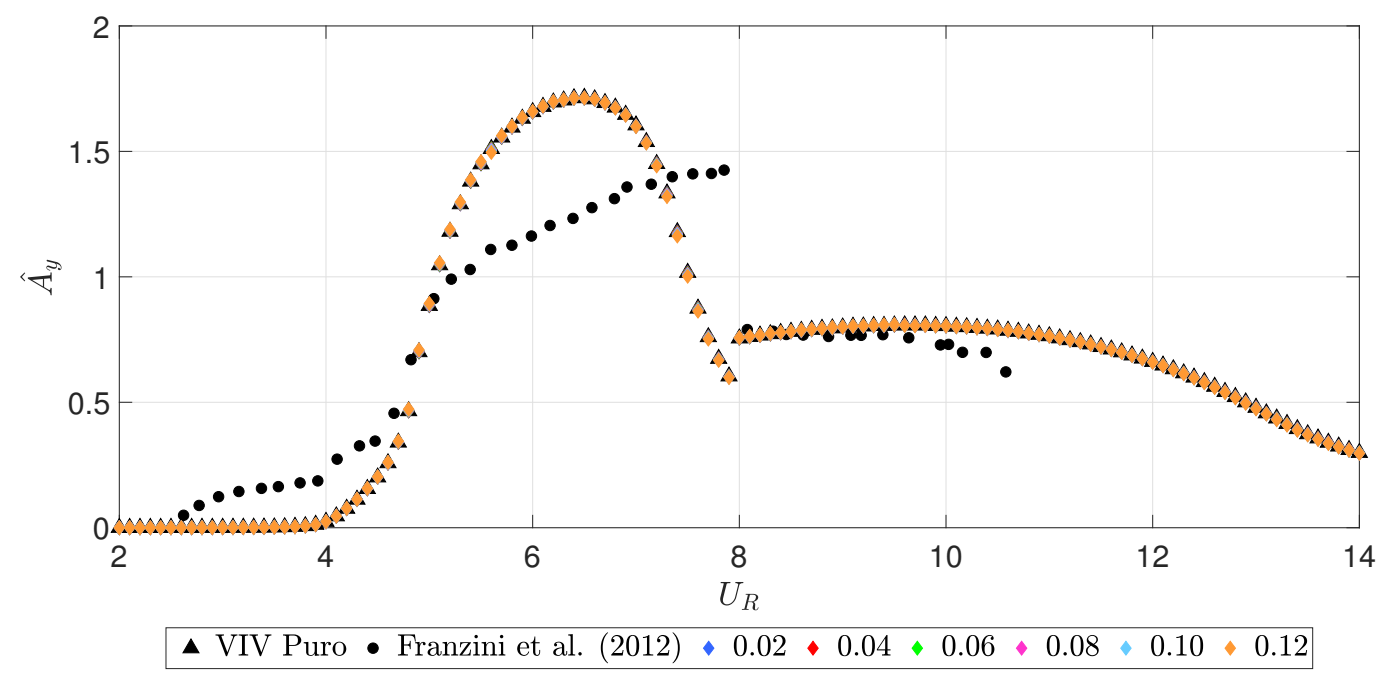

Fonte: O autor.

As amplitudes características de oscilação na direção longitudinal apresentadas na Figura 41 mostram que a excitação paramétrica promove um aumento da resposta. 
A amplificação das oscilações na direção in-line são mais evidentes para velocidades reduzidas superiores a 7,5. Além disso, nota-se que quanto maior a amplitude de excitação paramétrica $\delta$, maior é o valor da amplitude característica.

Figura 41 - Curvas de respostas da amplitude característica na direção in-line

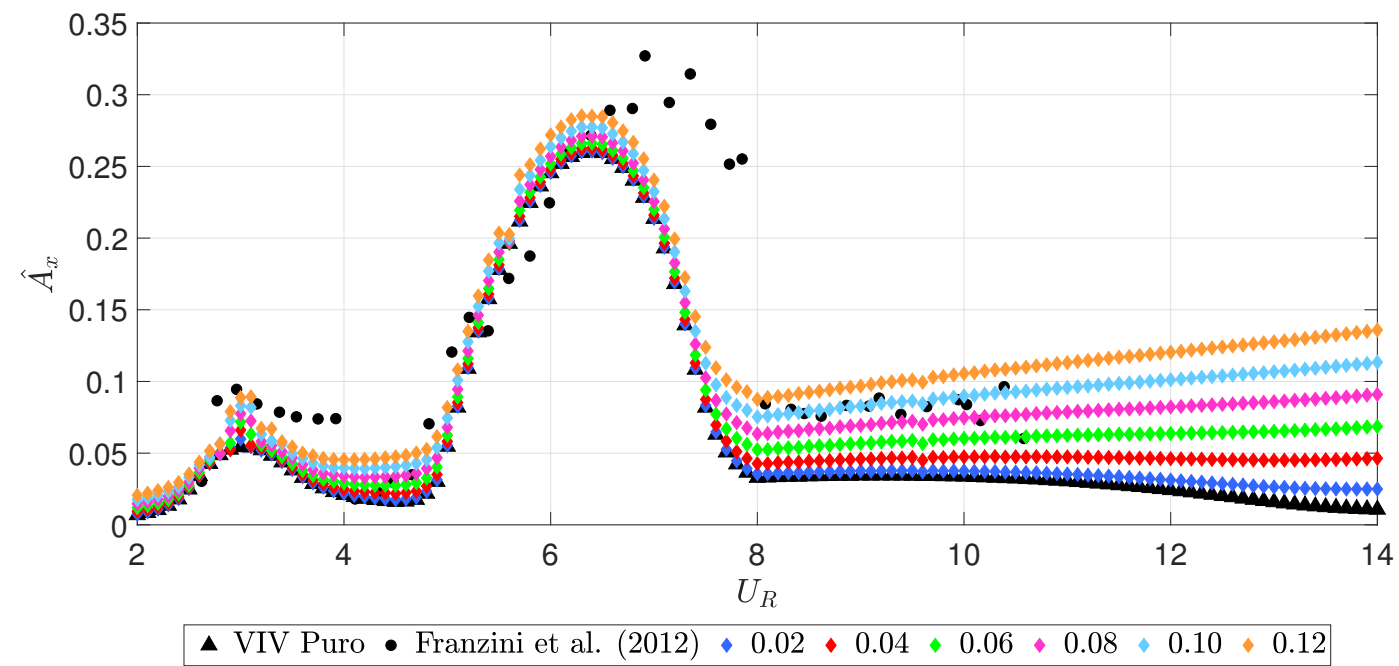

Fonte: O autor.

A Figura 42, apresenta as séries temporais de deslocamento do cilindro nas direções in-line e cross-wise, e os correspondentes espectros de amplitude para a condição "VIV Puro"com $U_{R}=5,5$ e $U_{R}=5,9$. Conforme pode ser observado na Figura 42, as séries temporais são livres de modulação em amplitude e possuem frequências dominantes iguais a $\hat{f} \approx 1,0$ na direção cross-wise e $\hat{f} \approx 2,0$ na direção in-line.

Figura 42 - Series temporais de resposta do cilindro para o caso sem excitação paramétrica
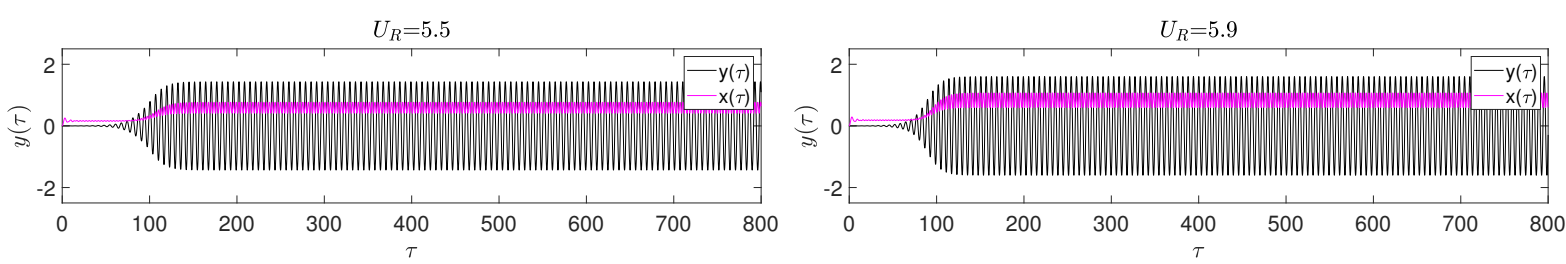

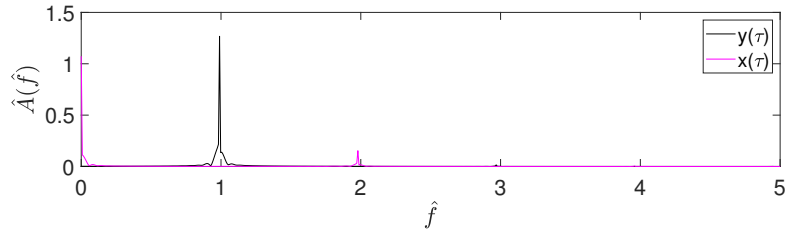

(a) $U_{R}=5,5$.

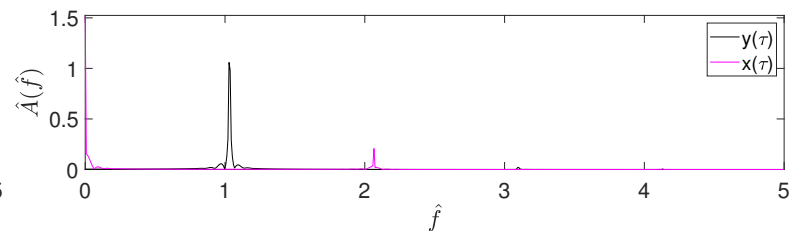

(b) $U_{R}=5,9$.

Fonte: O autor.

Na Figura 43 são apresentadas as séries temporais de deslocamento na condição VIV-2GL para as velocidades reduzidas $U_{R}=5,5$ e $U_{R}=5,9$, considerando o caso 
combinado de VIV e excitação paramétrica $\left(\delta_{y}=\delta_{x}=0,10\right)$. A Figura 43a mostra que a resposta do cilindro para a condição combinada é livre de modulação em amplitude nas duas direções e possuem espectro de amplitude semelhantes a condição de "VIV Puro".

Já na Figura 43b é possível perceber que existe uma pequena modulação em amplitude, em ambas as direções de oscilação. O espectro de amplitude da série temporal $y(\tau)$ sofre um pequeno alargamento da banda espectral e também possui frequência dominante $\hat{f} \approx 1,0$, enquanto que o espectro de amplitude da série temporal $x(\tau)$ tem uma frequência dominante pouco acima de $\hat{f} \approx 2,0$.

Figura 43 - Series temporais de resposta do cilindro para o caso $\delta_{y}=\delta_{x}=0,10 \mathrm{e}$ $n_{y}=n_{x}=1$
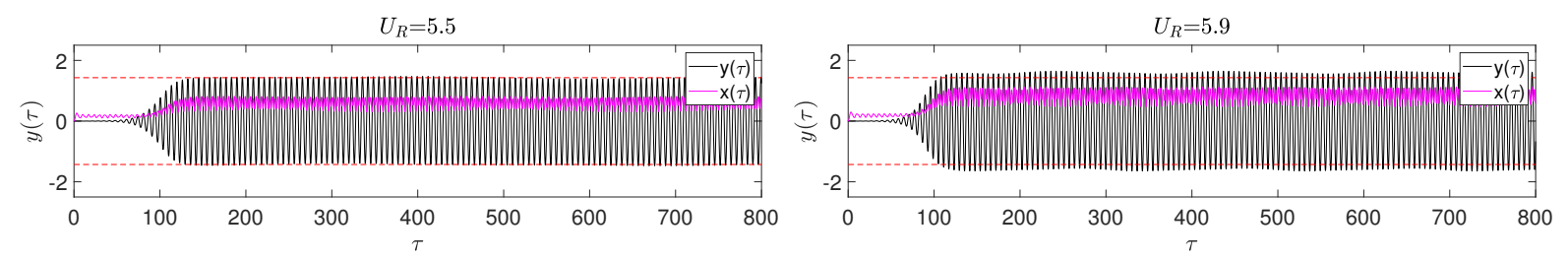

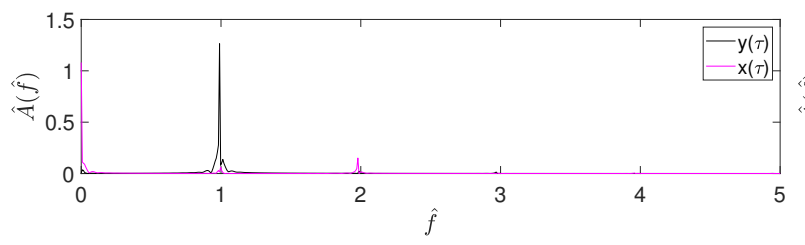

(a) $U_{R}=5,5$.

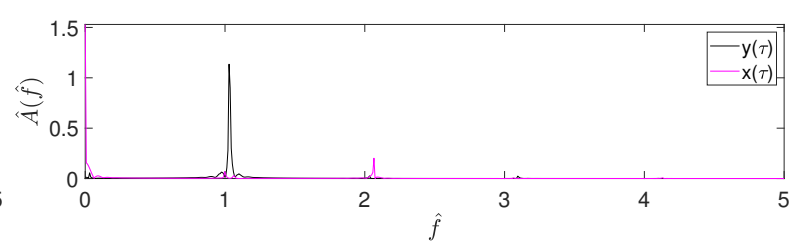

(b) $U_{R}=5,9$.

Fonte: O autor.

\subsubsection{Resultados considerando $n_{y}=n_{x}=2$}

As Figuras 44 e 45 apresentam as curvas de amplitude característica de oscilação do cilindro $\hat{A}_{y}\left(U_{R}\right)$ e $\hat{A}_{x}\left(U_{R}\right)$, respectivamente, variando as amplitudes de excitação paramétrica $\delta_{y}=\delta_{x}$ de 0,02 a 0,12 e considerando a frequência de excitação nas duas direções $n_{y}=n_{x}=2$.

De acordo com a Figura 44, a excitação paramétrica amplifica as amplitudes características somente no intervalo de velocidades reduzidas correspondente ao pico do lock-in $\left(5,5<U_{R} \leq 6,0\right)$. Do ponto de vista quantitativo, a amplitude máxima salta de $\max \left\{\hat{A}_{y}\right\} \approx 1,45$ ("VIV Puro") para $\max \left\{\hat{A}_{y}\right\} \approx 1,79$ (instabilidade paramétrica principal com $\left.\delta_{y}=\delta_{x}=0,10\right)$, o que representa um aumento de cerca de $24 \%$ na amplitude de oscilação na direção cross-wise. Comparando esse resultado com aquele obtido para o mesmo intervalo de velocidade reduzida e para condição VIV-1GL (ver Figura 23), nota-se que a amplificação das amplitudes características devida à excitação paramétrica é mais acentuada na condição VIV-1GL. 
Figura 44 - Curvas de respostas da amplitude característica na direção cross-wise

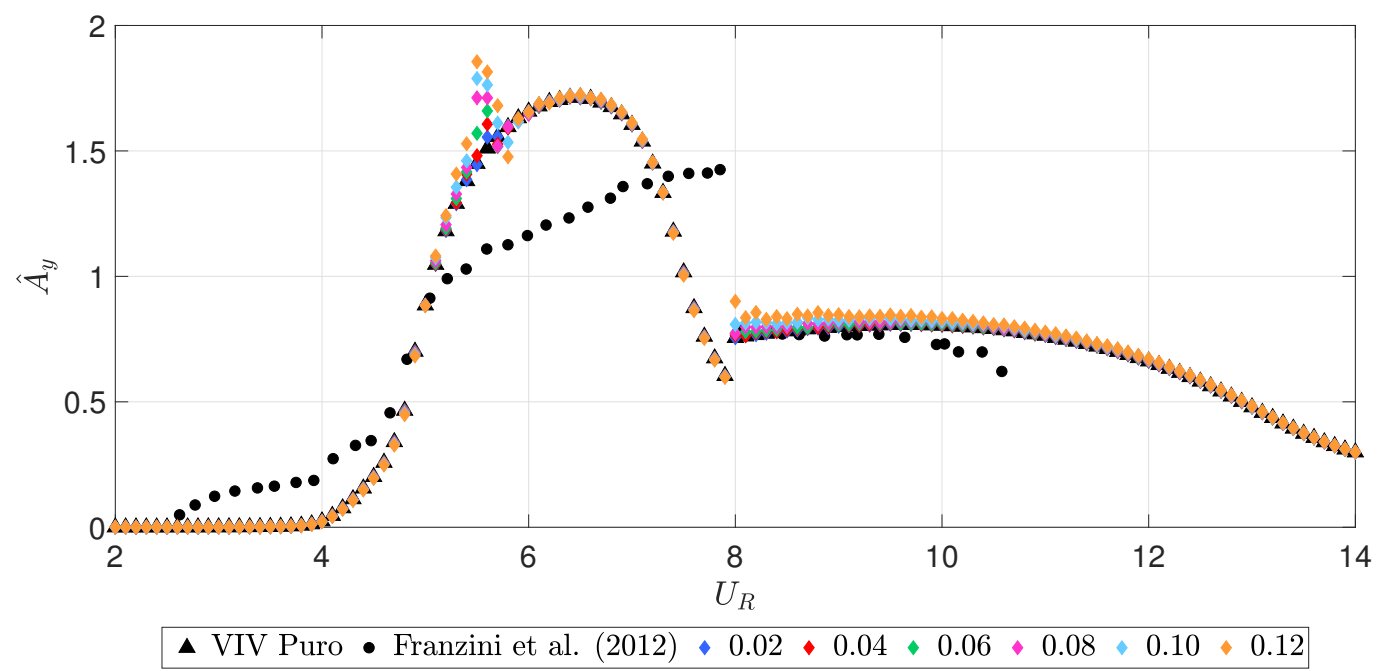

Fonte: O autor.

Já na Figura 45, a excitação paramétrica amplifica as amplitudes características em dois intervalos de velocidade reduzida distintos: o primeiro $2,5<U_{R} \leq 3,5$, e o segundo em 5,0 $<U_{R} \leq 7,5$, esse segundo intervalo é maior do que aquele do caso cross-wise. Além disso, é possível verificar que existe um pequeno pico de amplitude em torno de $U_{R} \approx 3,0$, o que esta relacionado a região de ressonância de VIV na direção longitudinal.

Figura 45 - Curvas de respostas da amplitude característica na direção in-line

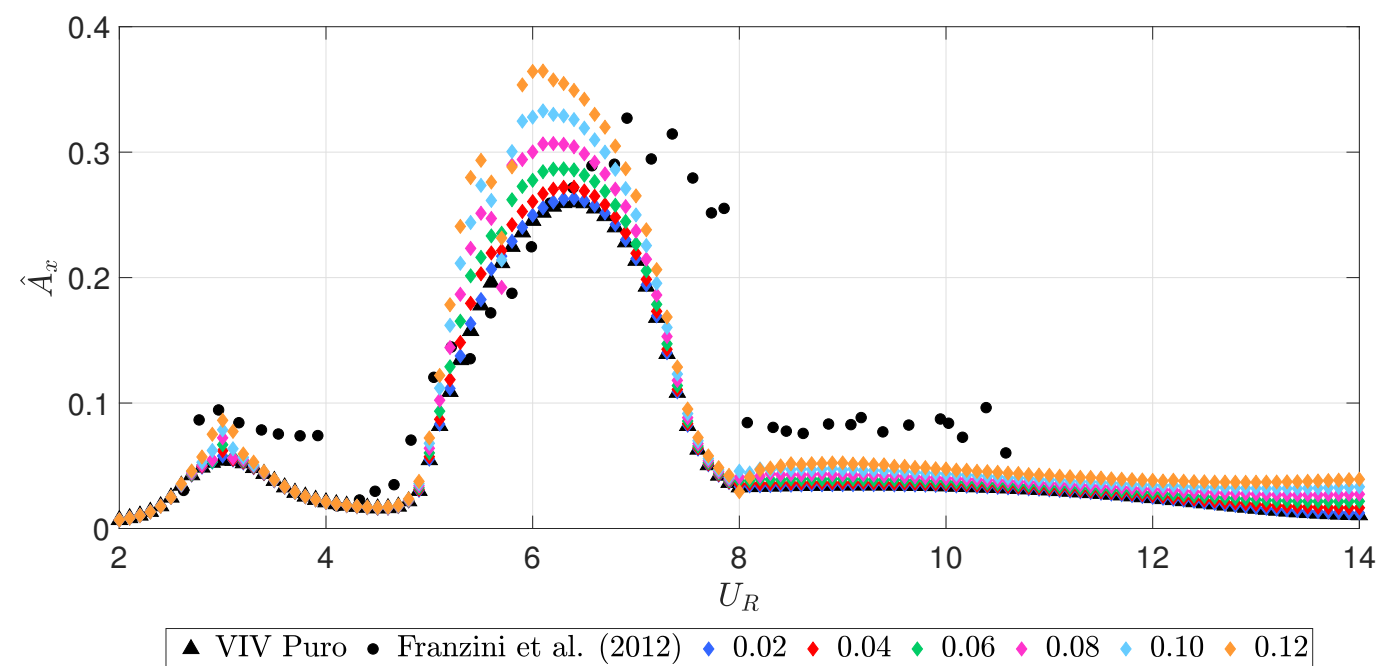

Fonte: O autor.

Pode-se verificar que do ponto de vista quantitativo, a amplitude máxima salta de $\max \left\{\hat{A}_{x}\right\} \approx 0,24$ ("VIV Puro") para $\max \left\{\hat{A}_{x}\right\} \approx 0,33$ (instabilidade paramétrica principal com $\left.\delta_{y}=\delta_{x}=0,10\right)$ em $U_{R}=6,0$. É interessante notar que, a partir de $U_{R}>8,0$, a 
excitação paramétrica não afeta a resposta do cilindro, diferente do que acontece no caso $n_{y}=n_{x}=1$ (ver Figura 41).

A Figura 46 ilustra as amplitudes características de oscilação na direção in-line com velocidade reduzida $U_{R} \approx 5,70$ do trabalho de Franzini et al. (2018). A condição "VIV Puro", mostrada na Figura 46a, aponta que os valores máximos de amplitude característica são $\max \left\{\hat{A}_{x}\right\} \approx 0,20$ e estão localizados pouco acima do meio do vão e correspondem ao segundo modo de vibrar. Para a condição combinada de VIV e excitação paramétrica $f_{t}: f_{N, 1}=2: 1$, vista na Figura $46 \mathrm{~b}$, é possível notar que a máxima amplitude característica de oscilação foi aumentada para $\max \left\{\hat{A}_{x}\right\} \approx 0,45$, devido a presença da excitação paramétrica. Esse resultado não possui mais a forma do segundo modo de vibrar, isso pode ocorrer devido a participação extras de outros modos de vibrar.

Figura 46 - Amplitude característica de oscilação direção na in-line

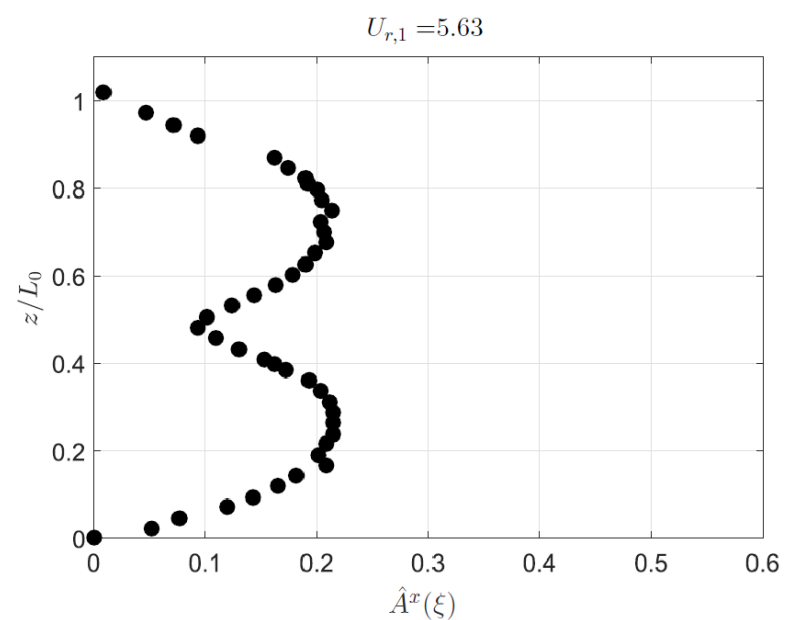

(a) "VIV Puro".

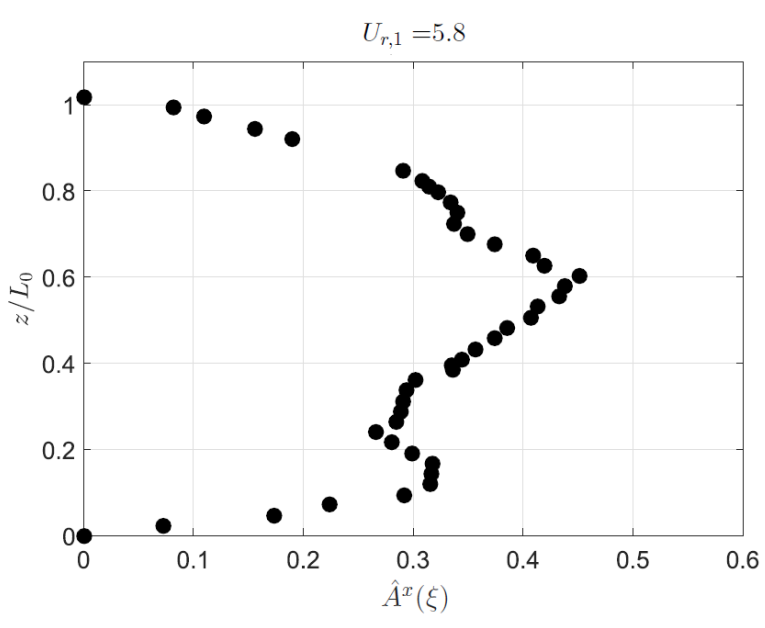

(b) $f_{t}: f_{N, 1}=2: 1$.

Fonte: Adaptado de Franzini et al. (2018).

Agora comparando os resultados experimentais de Franzini et al. (2018), mostrados na Figura 46b, com os resultados numéricos mostrados na Figura 45, percebe-se que para o caso combinado de VIV e excitação paramétrica o modelo fenomenológico pode recuperar qualitativamente um aumento da amplitude de oscilação em cerca de $30 \%$ considerando o caso de instabilidade paramétrica principal com $\delta_{y}=\delta_{x}=0,10$. Já no experimento de cilindro flexível houve um aumento da amplitude em cerca de $100 \%$.

As séries temporais de deslocamento do cilindro para o caso combinado de VIV e excitação paramétrica com $\delta_{y}=\delta_{x}=0,10$ e $n_{y}=n_{x}=2$ em $U_{R}=5,5$ e $U_{R}=5,9$ são respectivamente apresentadas na Figura 47. Analisando a série temporal da Figura 47a, o leitor nota que, a resposta do cilindro é livre de modulação em amplitude, sendo a amplitude característica aumenta de $\hat{A}_{y}=1,43$ ("VIV Puro") para $\hat{A}_{y}=1,76$ em $U_{R}=5,5$. Com relação aos espectro de amplitude, é possível notar que ambos são de banda estreita 
e possuem suas respectivas frequências adimensionais iguais a $\hat{f}=1,0$ e $\hat{f}=2,0$.

Como mostrado na Figura 47b, percebe-se que as séries temporais de deslocamento do cilindro possuem modulações em amplitude. Esse resultado é semelhante àquele ilustrado na Figura 25b para condição VIV-1GL. Além disso, é importante ressaltar que ambos espectros de amplitude apresentam um alargamento da banda espectral com frequências dominantes em $\hat{f}=1,0$ e $\hat{f}=2,0$.

Figura 47 - Series temporais de resposta do cilindro para o caso $\delta_{y}=\delta_{x}=0,10$ e $n_{y}=n_{x}=2$
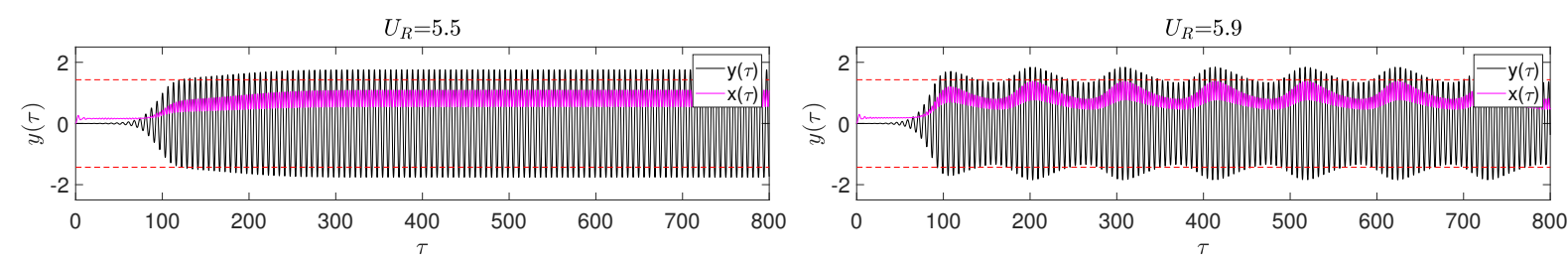

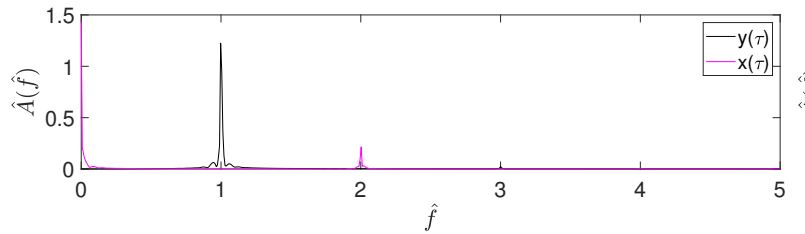

(a) $U_{R}=5,5$.

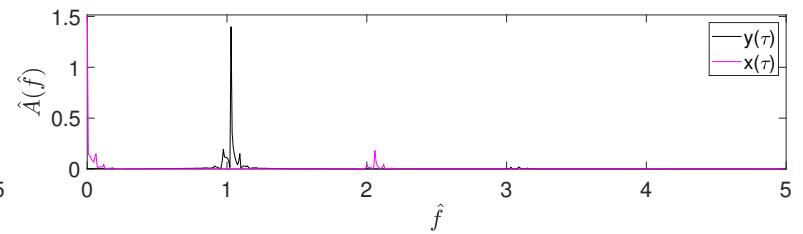

(b) $U_{R}=5,9$.

Fonte: O autor.

A Figura 48 apresenta as séries temporais de um ponto localizado próximo ao meio do vão obtidas por Franzini et al. (2018) na direção in-line para um cilindro flexível considerando $U_{R} \approx 5,70$. A Figura 48 a revela um comportamento com pouca modulação em amplitude e média não nula devido a força de arrasto que atua no modelo. Nota-se que o espectro de amplitude apresenta um pico de frequência dominante em $\hat{f} \approx 2,0$, que corresponde ao dobro da frequência na direção cross-wise (ver Figura 26a). Além disso, ainda é possível perceber a presença de outras componentes em frequência $\hat{f} \approx 1,0, \hat{f} \approx 3,0$ e $\hat{f} \approx 4,0$.

A Figura 48b mostra a série temporal obtida para o caso combinado de VIV e excitação paramétrica $f_{t}: f_{N, 1}=2: 1$. A resposta do cilindro flexível é caracterizada por uma forte modulação em amplitude, quando comparada a condição "VIV Puro". Entretanto, deve-se pontuar que o espectro de amplitude não sofre grandes alterações sob o efeito combinado.

\subsubsection{Coeficientes de força hidrodinamica VIV-2GL}

Na Figura 49 são apresentados os resultados numéricos-experimentais dos coeficientes de força obtidos para a configuração VIV-2GL, para diversos valores de amplitude de excitação paramétrica $\delta_{y}=\delta_{x}$ em $\overline{C_{x}}\left(U_{R}\right), C_{y, r . m . s}\left(U_{R}\right)$ e o $C_{x, r . m . s}\left(U_{R}\right)$. É importante 
Figura 48 - Series temporais de resposta do cilindro flexível
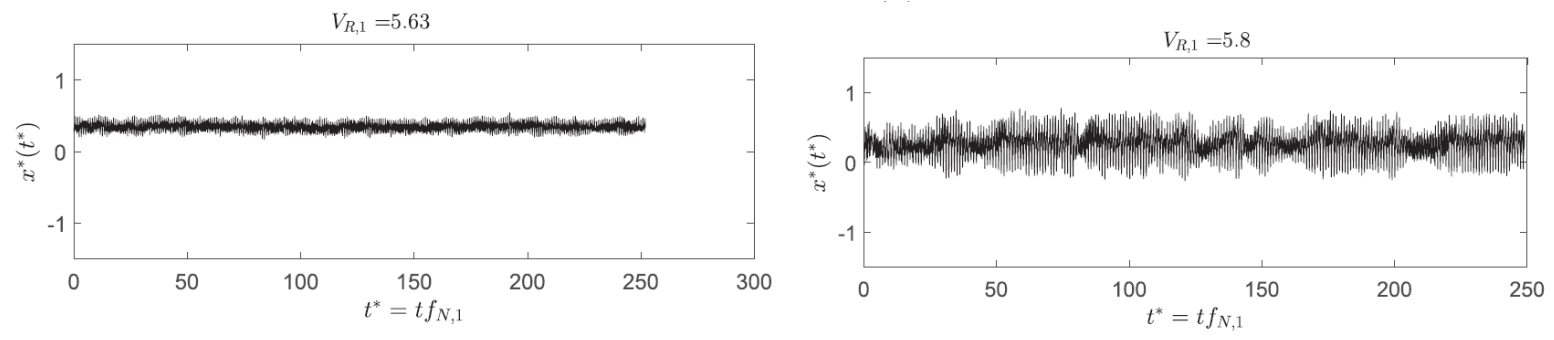

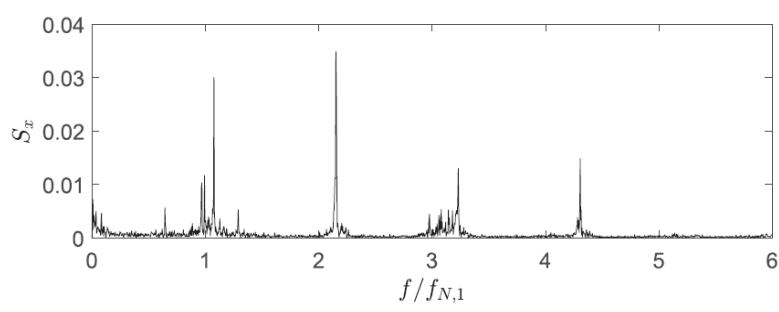

(a) "VIV Puro".

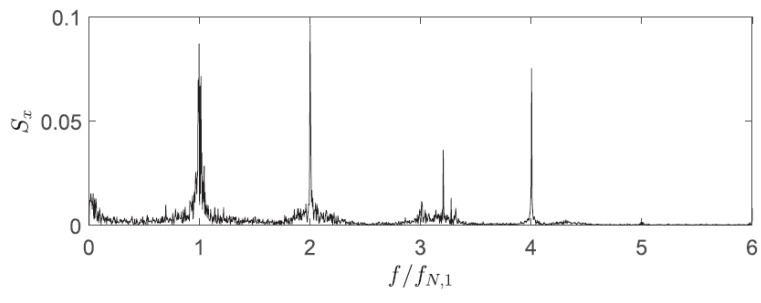

(b) $f_{t}: f_{N, 1}=2: 1$.

Fonte: Extraído de Franzini et al. (2018).

lembrar que, assim como foi mostrado na Subseção 4.1.3, os resultados experimentais apresentados em Franzini et al. (2012) foram feitos sob a condição "VIV Puro".

A Figura 49a revela que a excitação paramétrica pouco influi no coeficiente $\overline{C_{x}}$ durante o intervalo de lock-in. Já na Figura 49b, os coeficientes de força $C_{x, r . m . s}$ são amplificados para um pequeno intervalo de velocidades reduzidas $5,5<U_{R} \leq 6,0$. Comparando o resultado mostrado na Figura 49b com aquele do caso VIV-1GL (ver Figura 33c), percebe-se que para a condição VIV-1GL existe uma amplificação mais significativa das forças hidrodinâmicas no intervalo $4,5<U_{R} \leq 6,0$. Na análise da variação do coeficiente $C_{y, r . m . s}$, a Figura 49c mostra que o modelo fenomenológico apresenta um ligeiro aumento do valor máximo de $C_{y, r . m . s}$ durante o mesmo intervalo de velocidades reduzidas mostrado para os coeficientes $\overline{C_{x}}$ e $C_{x, r . m . s}$. Note que, durante o intervalo de velocidades reduzidas $2,0<U_{R} \leq 4,0$, os coeficientes de força força não foram qualitativamente recuperados pelo modelo fenomenológico.

Apresentados os resultados numéricos para as condições VIV-1GL e VIV-2GL, a próxima Subseção apresenta os resultados obtidos para uma investigação analítico-numérica usando o método de escalas múltiplas (MMS) na condição VIV-1GL. Essa investigação complementa os resultados numéricos da Subseção 4.1.2. 
Figura 49 - Influência da amplitude de excitação paramétrica nos coeficientes de força em relação à velocidade reduzida $U_{R}$

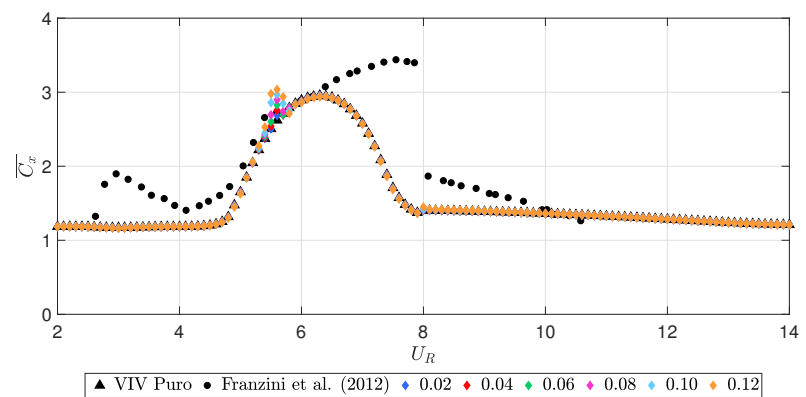

(a) $\overline{C_{x}}\left(U_{R}\right)$.

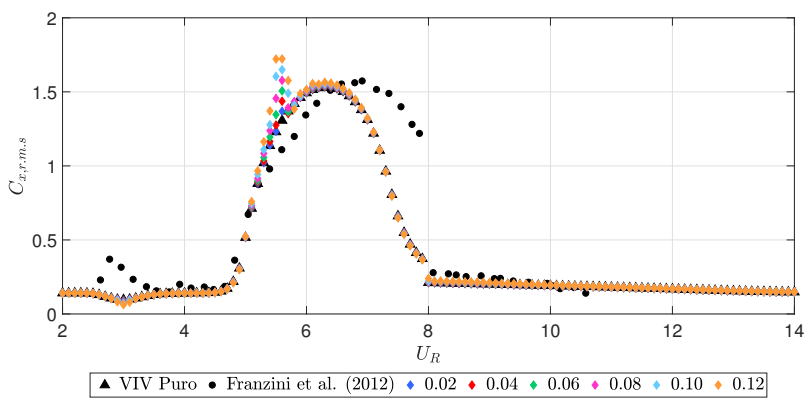

(b) $C_{x, r . m . s}\left(U_{R}\right)$.

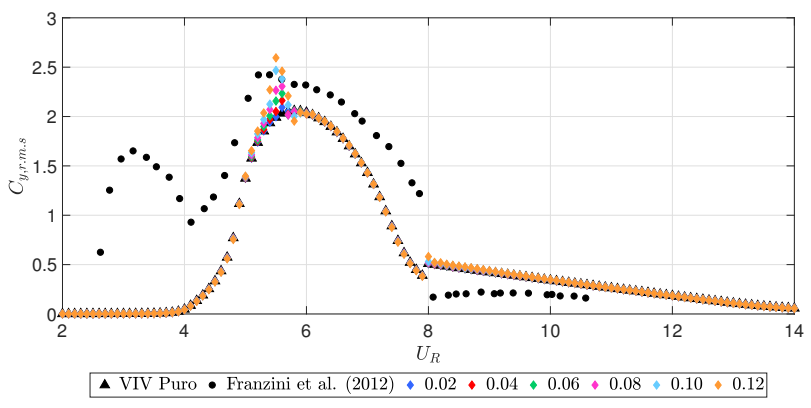

(c) $C_{y, r . m . s}\left(U_{R}\right)$.

Fonte: O autor.

\subsection{Solução assintótica utilizando o Método das Múltiplas Escalas - VIV 1-GL}

Para agilizar a obtenção da resposta do cilindro rígido montado em apoio elástico com um grau de liberdade, pode-se utilizar o método das múltiplas escalas como alternativa para a obtenção da solução numérica via método de Runge-Kutta. Apresenta-se aqui uma aproximação da solução do modelo hidro-elástico utilizando o método assintótica de pertubação das múltiplas escalas. Para utilizar esse método, é necessário introduzir diversas escalas de tempo na equação de movimento por meio de um pequeno parâmetro $\epsilon(0<\epsilon<<1)$. Aqui considera-se $\epsilon^{2} \beta_{1}=2 \zeta_{y}$ e $\epsilon^{2} \beta_{2}=\delta_{y}$. Então o modelo matemático é reescrito conforme visto nas Equações 4.1 e 4.2 .

$$
\begin{aligned}
& \ddot{y}+\epsilon^{2} \beta_{1} \dot{y}+\left(1+\epsilon^{2} \beta_{2} \cos \left(n_{y} \tau\right)\right) y= \\
& =a \epsilon^{2} q_{y}-b \epsilon^{2} \dot{y}+c \epsilon^{2} q_{y} \dot{y}^{2}-d \epsilon^{2} \dot{y}^{3} \\
& \ddot{q}_{y}+\epsilon^{2} g\left(q_{y}^{2}-1\right) \dot{q}_{y}+f^{2} q_{y}=\epsilon^{2} A_{y} \ddot{y}
\end{aligned}
$$


onde:

$$
\begin{aligned}
& a=\frac{U_{R}^{2} C_{L}^{0}}{4 \pi^{3}\left(m^{*}+C_{a}\right)}, b=\frac{U_{R} C_{D, V}}{\pi^{2}\left(m^{*}+C_{a}\right)}, c=\frac{C_{L}^{0}}{2 \pi\left(m^{*}+C_{a}\right)} \\
& d=\frac{2 C_{D, V}}{U_{R}\left(m^{*}+C_{a}\right)}, f^{2}=\left(S t U_{R}\right)^{2}, g=\epsilon_{y} S t U_{R}
\end{aligned}
$$

Foram utilizadas três escalas de tempo, $\tau_{0}=\tau, \tau_{1}=\epsilon \tau$ e $\tau_{2}=\epsilon^{2} \tau$ e as variáveis são expandidas como $y(\tau)=y_{0}\left(\tau_{0}, \tau_{1}, \tau_{2}\right)+\epsilon y_{1}\left(\tau_{0}, \tau_{1}, \tau_{2}\right)+\epsilon^{2} y_{2}\left(\tau_{0}, \tau_{1}, \tau_{2}\right)$ e $q_{y}(\tau)=$ $q_{y 0}\left(\tau_{0}, \tau_{1}, \tau_{2}\right)+\epsilon q_{y 1}\left(\tau_{0}, \tau_{1}, \tau_{2}\right)+\epsilon^{2} q_{y 2}\left(\tau_{0}, \tau_{1}, \tau_{2}\right)$. As derivadas do tempo com relação a $\tau$ e a família dos operadores diferenciais são fornecidas pelas Equações 4.5, 4.6 e 4.7.

$$
\begin{gathered}
\dot{(})=\frac{\partial}{\partial t}=D_{0}+\epsilon D_{1}+\epsilon^{2} D_{2}+\mathcal{O}\left(\epsilon^{3}\right) \\
\frac{\partial^{2}}{\partial t^{2}}=D_{0}^{2}+\epsilon D_{0} D_{1}+\epsilon^{2}\left(2 D_{0} D_{2}+D_{1}^{2}\right)+\mathcal{O}\left(\epsilon^{3}\right) \\
D_{n}^{k}=\frac{\partial_{k}}{\partial \tau^{k}}
\end{gathered}
$$

Substituindo os operadores diferenciais nas Equações 4.1 e 4.2 e coletando os termos de mesma ordem em $\epsilon$, são obtidos os seguintes sistemas de equações diferenciais ordinárias:

$$
\begin{gathered}
\mathcal{O}\left(\epsilon^{0}=1\right):\left\{\begin{array}{l}
D_{0}^{2} y_{0}+y_{0}=0 \\
D_{0}^{2} q_{y_{0}}+f^{2} q_{y_{0}}=0
\end{array}\right. \\
\mathcal{O}(\epsilon):\left\{\begin{array}{l}
D_{0}^{2} y_{1}+y_{1}=-2 D_{0} D_{1} y_{0} \\
D_{0}^{2} q_{y_{1}}+f^{2} q_{y_{1}}=-2 D_{0} D_{1} q_{y_{0}}
\end{array}\right. \\
\mathcal{O}\left(\epsilon^{2}\right):\left\{\begin{array}{l}
D_{0}^{2} y_{2}+y_{2}=-\beta_{1} D_{0} y_{0}-2 D_{0} D_{1} y_{1}-2 D_{0} D_{2} y_{2}-y_{0} \cos \left(n_{y} \tau\right) \beta_{2} \\
+a q_{y_{0}}-b D_{0} y_{0}+c D_{0}^{2} q_{y_{0}} y_{0}^{2}-d D_{0}^{3} y_{0}^{3}-D_{1}^{2} y_{0} \\
D_{0}^{2} q_{y_{2}}+f^{2} q_{y_{2}}=-2 D_{0} D_{1} q_{y_{1}}-2 D_{0} D_{2} q_{y_{0}}+g D_{0} y_{0}-g D_{0} q_{y_{0}}^{2}+A_{y} D_{0}^{2} y_{0}
\end{array}\right.
\end{gathered}
$$

A solução para a Equação 4.8 é bem conhecida e pode ser escrita como:

$$
\left\{\begin{array}{l}
y_{0}=R_{1}\left(\tau_{1}, \tau_{2}\right) e^{i \tau_{0}}+\text { c.c. } \\
q_{y_{0}}=R_{2}\left(\tau_{1}, \tau_{2}\right) e^{i f \tau_{0}}+\text { c.c. }
\end{array}\right.
$$


Onde "c.c."representa o complexo conjugado do termo anterior, i é a constante imaginária e $R_{1}, R_{2}$ são funções complexas em $\tau_{1}$ e $\tau_{2}$. Assim, define-se o termo referente a frequência de excitação paramétrica $n_{y}$ como:

$$
n_{y}=2+\epsilon^{2} \sigma
$$

com $\sigma$ sendo o parâmetro de dessintonia. Portanto, substituindo a Equação 4.11 na Equação 4.9, têm-se:

$$
\left\{\begin{array}{l}
D_{0}^{2} y_{1}+y_{1}=-2 D_{0} D_{1} R_{1}\left(\tau_{1}, \tau_{2}\right) e^{i \tau_{0}}+c . c . \\
D_{0}^{2} q_{y_{1}}+f q_{y_{1}}=-2 D_{0} D_{1} R_{2}\left(\tau_{1}, \tau_{2}\right) e^{i f^{2} \tau_{0}}+c . c .
\end{array}\right.
$$

Na Equação 4.13, os termos forçantes são ressonantes. Esses termos são conhecidos como termos seculares e geram soluções ilimitadas, precisando ser eliminados das soluções. Essa remoção é feita impondo condições de resolubilidade $D_{1} R_{1}=0$ e $D_{1} R_{2}=0$, como indicado nas Equações 4.14 e 4.15.

$$
\begin{aligned}
-2 i\left(D_{1} R_{1}\right) & =0 \\
-2 i f^{2}\left(D_{1} R_{2}\right) & =0
\end{aligned}
$$

O sistema de equações é reduzido de forma semelhante ao de primeira ordem $\mathcal{O}(1)$ e a solução de $y_{1}$ e $q_{y_{1}}$ são incluídas em $y_{0}$ e $q_{y_{0}}$. Seguindo, as Equações $\mathcal{O}\left(\epsilon^{2}\right)$ são:

$$
\begin{array}{r}
D_{0}^{2} y_{2}+y_{2}=-D_{0} y_{0} \beta_{1}-2 D_{0} D_{1} y_{1}-2 D_{0} D_{2} y_{2}-y_{0} \cos \left(\left(2+\epsilon^{2} \sigma\right) \tau\right) \beta_{2} \\
+a q_{y_{0}}-b D_{0} y_{0}+c D_{0}^{2} q_{y_{0}} y_{0}^{2}-d D_{0}^{3} y_{0}^{3}-D_{1}^{2} y_{0}
\end{array}
$$

Substituindo a Equação 4.11 nas Equações 4.16 e 4.17 e eliminando os termos seculares. Após um trabalho algébrico, as condições de resolubilidade são escritas como:

$$
\begin{array}{r}
-\frac{\beta_{2}}{2}\left(R_{1}^{*}\right) e^{i \sigma \tau_{2}}-i b R_{1}-i \beta_{1} R_{1}+a R_{2} e^{i \sigma_{1} \tau_{2}}-3 i d R_{1}^{2}\left(R_{1}^{*}\right) \\
+2 c e^{-i \sigma_{1} \tau_{2}} R_{1} R_{2}\left(R_{1}^{*}\right)-c e^{i \sigma_{1} \tau_{2}} R_{1}^{2}\left(R_{2}^{*}\right)-2 i\left(D_{2} R_{1}\right)=0 \\
-A_{y} e^{i \sigma_{1} \tau_{2}}+i f^{2} g R_{2}-i f^{2} g R_{2}^{2}\left(R_{2}^{*}\right)-2 i f^{2}\left(D_{2} R_{2}\right)=0
\end{array}
$$


Utilizando a forma polar de $R_{1}$ e $R_{2}$ resulta em:

$$
R_{1}=\frac{1}{2} \rho_{1}\left(\tau_{2}\right) e^{i \theta_{y}\left(\tau_{2}\right)} ; R_{2}=\frac{1}{2} \rho_{2}\left(\tau_{2}\right) e^{i \theta_{z}\left(\tau_{2}\right)}
$$

onde $\rho_{1}, \rho_{2}, \theta_{y}$ e $\theta_{z}$ são números reais e $\sigma_{1}$ é outro parâmetro de dessintonia. Substituindo a Equação 4.20 nas Equações 4.18 e 4.19 e separando as partes reais e imaginárias, o sistema de equações é escrito como:

$$
\begin{aligned}
& \frac{\beta_{2}}{4} \cos \left(\gamma_{1}\right) \rho_{1}-\frac{1}{2} \cos (\gamma) a \rho_{2}-\frac{1}{8} c \rho_{1}^{2} \rho_{2} \cos (\gamma)-\rho_{1}\left(D_{2} \theta_{y}\right)=0 \\
& \frac{\beta_{2}}{4} \sin \left(\gamma_{1}\right) \rho_{1}+\frac{1}{2} b \rho_{1}+\frac{1}{2} \beta_{1} \rho_{1}+\frac{3}{8} d \rho_{1}^{3}+\frac{1}{2} a \rho_{2} \sin (\gamma)+\frac{3}{8} c \rho_{1}^{2} \rho_{2} \sin (\gamma)+\left(D_{2} \rho_{1}\right)=0 \\
& \frac{1}{2} A_{y} \rho_{1} \cos (\gamma)-f^{2} \rho_{2}\left(D_{2} \theta_{z}\right)=0 \\
& \frac{1}{2} A_{y} \rho_{1} \sin (\gamma)-\frac{1}{2} f^{2} g \rho_{2}+\frac{1}{8} f^{2} g \rho_{2}^{3}+f^{2}\left(D_{2} \rho_{2}\right)=0 \\
& \quad \text { onde } \gamma \text { e } \gamma_{1} \text { são }
\end{aligned}
$$

$$
\gamma=\theta_{y}-\theta_{z}+\sigma_{1} \tau_{2} \quad ; \quad \gamma_{1}=2+\sigma \tau_{2}
$$

A Figura 50 mostra a comparação entre as séries temporais de deslocamento obtida a partir da simulação numérica com aquela obtida via solução assintótica das Equações 4.21-4.24. Os parâmetros utilizados no MMS estão presentes na Tabela 4.

Tabela 4 - Parâmetros utilizados no MMS.

\begin{tabular}{ll}
\hline Parâmetros & Valor \\
\hline$\epsilon$ & 0.1 \\
$\beta_{1}$ & 0.014 \\
$\beta_{2}$ & 10 \\
$\sigma$ & 0 \\
$\sigma_{1}$ & $\frac{\left(S t U_{R}-1\right)}{\epsilon^{2}}$ \\
$a$ & 2.89 \\
$b$ & 19.35 \\
$c$ & 1.70 \\
$d$ & 0.038 \\
$g$ & 0.325 \\
\hline
\end{tabular}


A partir da análise das séries temporais $y(\tau)$, é possível notar que os resultados obtidos via integração numérica das Equações 4.21-4.24 em ambiente MATHEMATICA ${ }^{\circledR}$, possuem boa concordância com os resultados obtidos a partir do modelo fenomenológico Equações 3.4 e 3.5. Nota-se, ainda, que a amplitude de modulação aparece em ambos resultados, semelhante ao resultado apresentado em Yuan, Xue e Tang (2018). O espectro de amplitude revela a dominância da frequência $\hat{f} \approx 1$. Também é importante ressaltar que o MMS pode capturar o enriquecimento do conteúdo espectral da resposta do cilindro. Além disso, houve um ganho computacional expressivo, pois o tempo de processamento do resultado via Runge-Kutta foi diminuído de 360s para apenas 5s utilizando a solução assintótica, justificando o emprego dessa técnica.

Figura 50 - Series temporais de resposta do cilindro utilizando o método das Múltiplas escalas para o caso VIV-1Gl e excitação paramétrica - $U_{R}=5,8$.
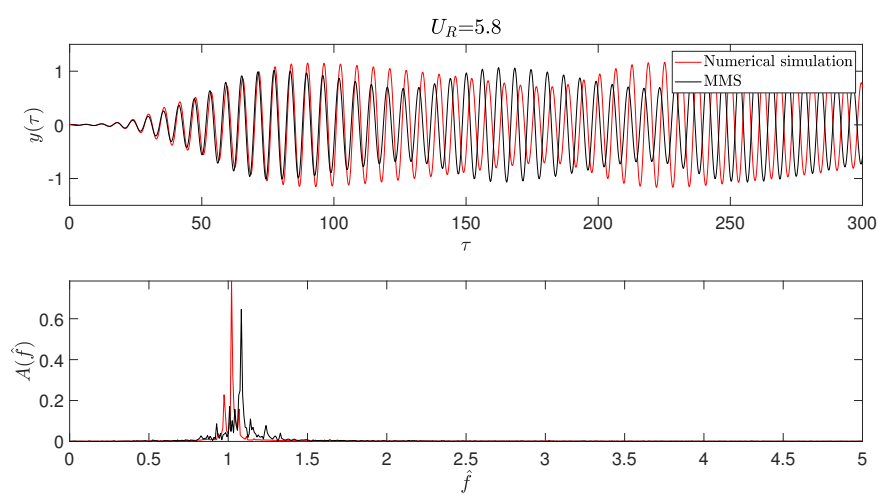

Fonte: O autor. 


\section{Considerações finais}

O objetivo deste trabalho foi estudar numericamente o fenômeno de vibração induzida pela emissão de vórtices (VIV) concomitantemente com a excitação paramétrica em cilindro rígido montado em apoio elástico. A excitação paramétrica foi considerada para o caso no qual a rigidez varia harmonicamente, enquanto o VIV foi investigado utilizando os modelos fenomenológicos de Ogink e Metrikine (2010) e Franzini e Bunzel (2018).

Este trabalho buscou preencher algumas lacunas identificadas ao final da revisão de literatura. Uma dessas lacunas remete ao estudo numérico de excitação paramétrica combinado ao fenômeno de VIV em cilindros rígidos montados em base elástica. Outra lacuna diz sobre o cálculo numérico da força hidrodinâmica no cilindro sujeito ao efeito combinado.

Inicialmente foi realizada uma revisão bibliográfica sobre os principais conceitos envolvendo o fenômeno de VIV e excitação paramétrica. Assim, pôde-se desenvolver o equacionamento necessário para modelar o problema de cilindros rígidos montados em apoio elástico nas condições VIV-1GL (livre para oscilar somente na direção transversal) e VIV-2GL (livre para oscilar nas direções transversal e longitudinal). As contribuições aqui apresentadas foram a comparação crítica entre a condição investigada e os resultados obtidos numericamente e experimentalmente de cilindros flexíveis, destacando aspectos qualitativos e quantitativos recuperados pelos modelos de ordem reduzida.

As principais observações são resumidas da seguinte forma. Para a condição VIV$1 \mathrm{GL}$, os resultados numéricos da variação da amplitude de oscilação para o caso combinado de VIV e excitação paramétrica, considerando a frequência de excitação paramétrica $\left(n_{y}=1\right)$, não foram alterados durante todo intervalo de lock-in. Já no modelo flexível percebeu-se que a excitação paramétrica amplificou a resposta se comparada ao caso excitado somente pelo VIV. Considerando o caso de instabilidade paramétrica principal $\left(n_{y}=2\right)$, houve um aumento na amplitude de oscilação do cilindro e dos coeficientes de força somente em um intervalo específico da região de lock-in $\left(4,5 \leq U_{R} \leq 6,0\right)$. No entanto, no cilindro flexível, essa variação pode ocorrer fora do intervalo de lock-in. Comparando o coeficiente de arrasto médio $\left(\overline{C_{x}}\right)$ na região lock-in do cilindro flexível com o cilindro rígido, notou-se que o modelo fenomenológico pode recuperar qualitativamente o aumento do coeficiente de arrasto, como observado no experimento e resultados numéricos de cilindros flexíveis.

Ainda para a condição VIV-1GL, aplicou-se o método das múltiplas escalas (MMS) com o intuito de obter uma solução assintótica do problema combinado de VIV e excitação paramétrica. Foram recuperadas as mesmas características observadas na integração 
numérica das equações de movimento. Ressalta-se que o MMS diminui o tempo de processamento na obtenção da resposta, podendo se tornar um fator interessante em voltas iniciais de um projeto. Pelo menos até onde é do conhecimento do autor, as análises por MMS sobre esse tipo de problema não são encontradas na literatura e consistem em uma nova contribuição desta pesquisa.

Convém salientar que foram encontradas modulações de amplitude na série temporal de deslocamento para o caso combinado, uma característica também observada experimentalmente para o cilindro flexível sob VIV e excitação paramétrica. Os resultados mostrados nesta pesquisa e em outras referências da literatura indicam que o uso da análise do espectro de amplitude é complementado com a transformada de Hilbert-Huang, pois foi possível notar claramente a modulação de frequência existente. Além disso, foi possível perceber, por meio da investigação do espectro de amplitude para o caso combinado, a presença de espectros de banda larga centrados em $\hat{f}=f / f_{N, y}=1$.

Agora no que se refere ao problema VIV-2GL, as análises realizadas das curvas de amplitude de oscilação e os coeficientes de força em função da velocidade reduzida, foram pouco alteradas para o caso de instabilidade paramétrica principal ( $n_{y}=n_{x}=2$ ) no intervalo de lock-in $\left(5,5 \leq U_{R} \leq 6,0\right)$. Dessa forma, comparando os resultados numéricos obtidos para a condição VIV-2GL com os resultados experimentais de cilindro flexível, notou-se que o modelo fenomenológico não conseguiu recuperar o aumento da resposta do cilindro para velocidades reduzidas fora do pico de lock-in.

Trabalhos futuros podem incluir extensão desta pesquisa utilizando modelos fenomenológicos em cilindros flexíveis submetidos à mesmas combinações de carregamentos. Além disso, podem ser realizadas simulações numéricas utilizando dinâmica de fluidos computacionais (DFC) considerando cilindros rígidos montados em base elástica, o que já é suficientemente desafiador. É importante ressaltar também que dados experimentais para correlação direta com os resultados aqui apresentados não foram encontrados na literatura. Isso se deve à difícil tarefa de criar um experimento no qual um cilindro rígido é montado em apoio elástico cuja rigidez varia harmonicamente com o tempo. 


\section{Referências}

ASSI, G. R. d. S. Estudo experimental do efeito de interferência no escoamento ao redor de cilindros alinhados. Dissertação (Mestrado) - Escola Politécnica, Universidade de São Paulo, 2005.

ASSI, G. R. S. Mechanisms for flow-induced vibration of interfering bluff bodies. Tese (Doutorado) — Imperial College London, 2009.

BEARMAN, P. W. Vortex shedding from oscillating bluff bodies. Annual Review of Fluid Mechanics, v. 16, p. 195-222, jan. 1984.

BEARMAN, P. W. Circular cylinder wakes and vortex-induced vibrations. Journal of Fluids and Structures, v. 27, p. 648-658, 072011.

BIRKHOFF, G. Formation of vortex streets. Journal of applied physics, v. 24, n. 1, p. 98-103, jan. 1953.

BISHOP, R. E. D.; HASSAN, A. Y. The lift and drag forces on a circular cylinder oscillating in a flowing fluid. Royal Society London, p. 277: 51-75, 1964.

BLEVINS, R. D.; COUGHRAN, C. S. Experimental investigation of vortex-induced vibration in one and two dimensions with variable mass, damping, and reynolds number. Journal of Fluids Engineering, ASME International, v. 131, n. 10, sep 2009.

BUTIKOV, E. Parametric resonance. Computing in Science \& Engineering, Institute of Electrical and Electronics Engineers (IEEE), v. 1, n. 3, p. 76-83, 1999.

CHATJIGEORGIOU, I.; MAVRAKOS, S. Bounded and unbounded coupled transverse response of parametrically excited vertical marine risers and tensioned cable legs for marine applications. Applied Ocean Research, Elsevier BV, v. 24, n. 6, p. 341-354, dec 2002.

CUNHA, L. D. Vibração induzida por vórtices: análise crítica de modelos fenomenológicos. Dissertação (Mestrado) — Escola Politécnica, Universidade de São Paulo, 2005.

FACCHINETTI, M. L.; de LANGRE, E.; BIOLLEY, F. Coupling of structure and wake oscillators in vortex-induced vibrations. Journal of Fluids and Structures, v. 19, p. 123-140, 2004.

FENG, C. The measurement of vortex induced effects in flow past stationary and oscillating circular and d-section cylinders. University of British Columbia, 1968.

FRANZINI, G.; MAZZILLI, C. Non-linear reduced-order model for parametric excitation analysis of an immersed vertical slender rod. International Journal of Non-Linear Mechanics, Elsevier BV, v. 80, p. 29-39, apr 2016.

FRANZINI, G. R. Tópicos de pesquisa em problemas de excitação paramétrica e de vibrações induzidas pelo escoamento paramétrica e de vibrações induzidas pelo escoamento. 2019. Universidade de São Paulo, Escola Politécnica - Tese (livre-docência). 
FRANZINI, G. R.; BUNZEL, L. O. A numerical investigation on piezoelectric energy harvesting from vortex-induced vibrations with one and two degrees of freedom. Journal of Fluids and Structures, Elsevier BV, v. 77, p. 196-212, 2018.

FRAnZINI, G. R.; GONALVES, R. T.; MENEGHINI, J. R.; FUJARRA, A. L. C. Comparison between force measurements of one and two degrees-of-freedom viv on cylinder with small and large mass ratio. In: Proceedings of the 10th FIV 2012 International Conference on Flow-Induced Vibrations Conference (\& Flow-Induced Noise). [S.l.: s.n.], 2012.

FRANZINI, G. R.; GONÇALVES, R. T.; PESCE, C. P.; FUJARRA, A. L. C.; MAZZILLI, C. E. N.; MENEGHINI, J. R.; MENDES, P. Vortex-induced vibration experiments with a long semi-immersed flexible cylinder under tension modulation: Fourier transform and Hilbert-Huang spectral analyses. Journal of the Brazilian Society of Mechanical Sciences and Engineering, v. 37, n. 2, p. 589-599, 2015.

FRANZINI, G. R.; PESCE, C. P.; GONÇALVES, R. T.; FUJARRA, A.; MENDES, P. Experimental investigations on vortex-induced vibrations with a long flexible cylinder. part ii: Effect of axial motion excitation in a vertical configuration. In: Proceedings of the 11th FIV 2016 - International Conference on Flow-Induced Vibrations Conference (E) Flow-Induced Noise). [S.l.: s.n.], 2016.

FRAnZINI, G. R.; PESCE, C. P.; GONÇALVES, R. T.; FUJARRA, A. L. C.; MENDES, P. An experimental investigation on concomitant vortex-induced vibration and axial top-motion excitation with a long flexible cylinder in vertical configuration. Ocean Engineering, Elsevier BV, v. 156, p. 596-612, maio 2018.

FUJARRA, A. L. C.; PESCE, C. P.; Flemming, F.; WilliamsOn, C. H. K. Vortex-induced vibration of a flexible cantilever. Journal of Fluids and Structures, Elsevier BV, v. 15, n. 3-4, p. 651-658, 2001.

GERRARD, J. H. The mechanics of the formation region of vortices behind bluff bodies. Journal of Fluid Mechanics, Cambridge University Press (CUP), v. 25, n. 02, p. 401-413, jun. 1966.

GONÇALVES, R. T.; FRANZINI, G. R.; ROSETTI, G. F.; FUJARRA, A. L. C.; NISHIMOTO, K. Analysis methodology for vortex-induced motions (VIM) of a monocolumn platform applying the Hilbert-Huang transform method. Journal of Offshore Mechanics and Arctic Engineering, v. 113, p. 011103-1 - 011103-7, 2012.

GOVARDHAN, R.; WILLIAMSON, C. H. K. Modes of vortex formation and frequency response of a freely vibrating cylinder. Journal of Fluid Mechanics, v. 420, p. 85-130, 2000 .

HUANG, N. E.; SHEN, Z.; LONG, S. R.; WU, M. C.; SHIH, H. H.; ZHENG, Q.; YEN, N.; TUNG, C. C.; LIU, H. H. The empirical mode decomposition and the Hilbert spectrum for nonlinear and non-stationary time series analysis. Royal Society London, v. 454, p. 903-955, 1998.

IWAN, W. D.; BLEVINS, R. D. A model for vortex-induced oscillation of structures. Journal of Applied Mechanics, v. 41, p. 581-586, 1974. 
JAUVTIS, N.; WILLIAMSON, C. H. K. The effect of two degrees of freedom on vortex-induced vibration at low mass and damping. Journal of Fluid Mechanics, Cambridge University Press (CUP), v. 509, p. 23-62, jun 2004.

KHALAK, A.; WILLIAMSON, C. H. K. Motions, forces and modes transitions in Vortex-Induced Vibration at low Reynolds number. Journal of Fluids and Structures, v. 13, p. 813-851, 1999.

LIENHARD, J. H. Synopsis of lift, drag, and vortex frequency data for rigid circular cylinders. Washington: Pullman, 1966.

LIU, G.; LI, H.; QIU, Z.; LENG, D.; LI, Z.; LI, W. A mini review of recent progress on vortex-induced vibrations of marine risers. Ocean Engineering, Elsevier BV, v. 195, p. 106704, jan 2020.

MA, Y.; XU, W.; PANG, T.; WANG, Q.; LAI, J. Dynamic characteristics of a slender flexible cylinder excited by concomitant vortex-induced vibration and time-varying axial tension. Journal of Sound and Vibration, Elsevier BV, p. 115524, jul 2020.

MAZZILLI, C. E.; RIZZA, F.; DIAS, T. Heave-imposed motion in vertical risers: A reduced-order modelling based on bessel-like modes. Procedia IUTAM, Elsevier BV, v. 19, p. 136-143, 2016.

MEIROVITCH, L. Methods of Analytical Dynamics. Mineola: Dover Publications, 2003.

NAYFEH, A. H.; MOOK, D. T. Nonlinear oscillations. Weinheim: John Wiley \& Sons, 1979. ISBN 9783527617586.

NORBERG, C. Flow around a circular cylinder: aspects of fluctuating lift. Journal of Fluids and Structures, Elsevier BV, v. 15, n. 3-4, p. 459-469, abr. 2001.

OGINK, R.; METRIKINE, A. A wake oscillator with frequency dependent coupling for the modeling of vortex-induced vibration. Journal of Sound and Vibration, Elsevier BV, v. 329 , n. 26 , p. 5452-5473, dez. 2010.

PATEL, M.; PARK, H. Dynamics of tension leg platform tethers at low tension. part i mathieu stability at large parameters. Marine Structures, Elsevier BV, v. 4, n. 3, p. 257-273, jan 1991.

PESCE, C. P.; FUJARRA, A. L. C. Vortex-induced vibrations and jump phenomenon: Experiments with a clamped flexible cylinder in water. International Journal of Offshore and Polar Engineering, v. 10, p. 26-33, 2000.

PESCE, C. P.; FUJARRA, A. L. C. The "super-upper branch" viv response of flexible cylinders. In: BBVIV4. The "super-upper branch" VIV response of flexible cylinders. [S.1.], 2005. v. 4.

PESCE, C. P.; FUJARRA, A. L. C.; FRANZINI, G. R.; RATEIRO, F.; GONALVES, R. T.; GONçALVES, R. T.; SALLES, R. Dinâmica não-linear de risers: Experimentos Hidro-elásticos com cilindros flexíveis verticais em tanque de reboque IPT. [S.l.], 2013.

PESCE, C. P.; FUJARRA, A. L. C.; KUBOTA, L. K. The hilbert-huang spectral analysis method applied to VIV. In: Proceedings of the OMAE2006 25th International Conference on Offshore Mechanics and Arctic Engineering. Hamburgo: ASME, 2006. 
PONCET, R. Dynamic analysis of oshore risers. [S.l.], 2011. Universidade de São Paulo, Escola Politécnica - Scientific internship report.

POSTNIKOV, A.; PAVLOVSKAIA, E.; WIERCIGROCH, M. 2dof CFD calibrated wake oscillator model to investigate vortex-induced vibrations. International Journal of Mechanical Sciences, Elsevier BV, v. 127, p. 176-190, jul 2017.

QU, Y.; METRIKINE, A. V. A single van der pol wake oscillator model for coupled cross-flow and in-line vortex-induced vibrations. Ocean Engineering, Elsevier BV, v. 196, p. 106732, jan 2020 .

SARPKAYA, T. A critical review of the intrinsic nature of vortex-induced vibrations. Journal of Fluids and Structures, v. 19, p. 389-447, 2004.

SILVEIRA, L. M. Y. da; MARTINS, C. de A.; CUNHA, L. D.; PESCE, C. P. An investigation on the effect of tension variation on VIV of risers. In: Volume 1: Offshore Technology + Special Symposium on Ocean Measurements and Their Influence on Design. [S.1.]: ASMEDC, 2007.

SIMOS, A. N.; PESCE, C. P. Mathieu stability in the dynamics of tlp's tethers considering variable tension along the length. In: Transactions on the Built Enviroment. [S.l.: s.n.], 1997. v. 29.

SKOP, R.; GRIFFIN, O. A model for the vortex-excited resonant response of bluff cylinders. Journal of Sound and Vibration, Elsevier BV, v. 27, n. 2, p. 225-233, mar. 1973.

SRINIL, N.; ZANGANEH, H. Modelling of coupled cross-flow/in-line vortex-induced vibrations using double duffing and van der pol oscillators. Ocean Engineering, Elsevier BV, v. 53, p. 83-97, oct 2012.

THAMPI, S. K.; NIEDZWECKI, J. M. Parametric and external excitation of marine risers. Journal of Engineering Mechanics, American Society of Civil Engineers (ASCE), v. 118, n. 5, p. 942-960, maio 1992.

UENO, T.; FRANZINI, G. R. Numerical studies on passive suppression of one and two degrees-of-freedom vortex-induced vibrations using a rotative non-linear vibration absorber. International Journal of Non-Linear Mechanics, Elsevier BV, v. 116, p. 230-249, nov 2019.

VERNIZZI, G. J.; FRANZINI, G. R.; LENCI, S. Reduced-order models for the analysis of a vertical rod under parametric excitation. International Journal of Mechanical Sciences, Elsevier BV, v. 163, p. 105122, nov 2019.

WILLIAMSON, C.; GOVARDHAN, R. Vortex-induced vibrations. Annual Review of Fluid Mechanics, Annual Reviews, v. 36, n. 1, p. 413-455, jan. 2004.

WILLIAMSON, C.; GOVARDHAN, R. A brief review of recent results in vortex-induced vibrations. Journal of Wind Engineering and Industrial Aerodynamics, Elsevier BV, v. 96, n. 6-7, p. 713-735, jun 2008.

WILLIAMSON, C. H. K.; ROSHKO, A. Vortex formation in the wake of an oscillating cylinder. Journal of Fluid Mechanics, v. 2, p. 355-381, 1988. 
WU, X.; GE, F.; HONG, Y. A review of recent studies on vortex-induced vibrations of long slender cylinders. Journal of Fluids and Structures, Elsevier BV, v. 28, p. 292-308, jan 2012 .

YUAN, Y.; XUE, H.; TANG, W. A numerical investigation of vortex-induced vibration response characteristics for long flexible cylinders with time-varying axial tension. Journal of Fluids and Structures, Elsevier BV, v. 77, p. 36-57, fev. 2018.

ZHANG, J.; GUO, H.; TANG, Y.; LI, Y. Effect of top tension on vortex-induced vibration of deep-sea risers. Journal of Marine Science and Engineering, MDPI AG, v. 8 , n. 2, p. 121, feb 2020 . 